\title{
A Critical Compilation of Energy Levels, Spectral Lines, and Transition Probabilities of Singly Ionized Silver, Ag II
}

\author{
Alexander Kramida \\ National Institute of Standards and Technology, \\ Gaithersburg, MD 20899 \\ alexander.kramida@nist.gov
}

\begin{abstract}
All available experimental measurements of the spectrum of the $\mathrm{Ag}^{+}$ion are critically reviewed. Systematic shifts are removed from the measured wavelengths. The compiled list of critically evaluated wavelengths is used to derive a comprehensive list of energy levels with well-defined uncertainties. Eigenvector compositions and level designations are found in two alternate coupling schemes. Some of the older work is found to be incorrect. A revised value of the ionization energy, 173283(7) $\mathrm{cm}^{-1}$, equivalent to $21.4844(8) \mathrm{eV}$, is derived from the new energy levels. A set of critically evaluated transition probabilities is given.
\end{abstract}

Key words: atomic energy levels; critically evaluated data; ionization limit; singly ionized silver; transition probabilities; wavelengths.

Accepted: April 2, 2013

Published: April 15, 2013

http://dx.doi.org/10.6028/jres.118.009

\section{Introduction}

Ag II, like the isoelectronic neutral palladium, has the ground term $4 \mathrm{~d}^{10}{ }^{1} \mathrm{~S}_{0}$. Promotion of one electron from the $4 \mathrm{~d}$ shell produces a relatively simple system of level series $4 \mathrm{~d}^{9} \mathrm{nl}$. Promotion of two electrons results in complex configurations $4 d^{8} n l n^{\prime} l^{\prime}$ with three open shells. Two of them, $4 d^{8} 5 s^{2}$ and $4 d^{8} 5 s 5 p$, appear below the ionization limit and contribute to the complexity of the observed spectrum.

The most complete previous analysis of the Ag II spectrum was made by Kalus et al. [1]. These authors measured the spectrum emitted by a pulsed hollow-cathode discharge in the region $940 \AA$ to $8500 \AA$. A good review of the previous work can be found in that paper.

Kalus et al. [1] noted that their line list does not include many of the lines reported by earlier observers. In particular, they mentioned that Gilbert [2] was able to observe many more combinations between highly excited levels by using a condensed hollow-cathode discharge. Rasmussen [3,4] reported 40 lines originating from the $4 \mathrm{~d}^{9} 6 \mathrm{p}, 4 \mathrm{~d}^{8} 5 \mathrm{~s}^{2}$, and $4 \mathrm{~d}^{8} 5 \mathrm{~s} 5 \mathrm{p}$ configurations that were not observed by Kalus et al. Benschop et al. [5] listed 65 lines ascribed to Ag II, 43 of which they classified as transitions involving the highly excited $4 \mathrm{~d}^{9} 8 \mathrm{~s}$ and $4 \mathrm{~d}^{9} 9 \mathrm{~s}$ configurations. They used two different types of spark discharges as light sources, a sliding spark and a three-electrode vacuum spark. Kalus et al. [1] suggested the use of these spark sources as the reason for the appearance of lines from highly excited levels in the spectra observed by Benschop et al. [5]. However, other authors who used spark discharges [6,7] have identified lines from only moderately excited configurations $\left(4 d^{9} 5 s, 4 d^{9} 6 s, 4 d^{9} 5 p, 4 d^{9} 5 d\right)$. The latter two works contain 32 lines not observed by Kalus et al. 
Benschop et al. [5] based their line identifications and new level values on their own measurements and those previously made by Shenstone [6] and Gilbert [2]. Neither of these measurements was especially precise. For example, for strong unblended lines, uncertainties of wave numbers measured by Benschop et al. [5] were $0.13 \mathrm{~cm}^{-1}$ to $0.5 \mathrm{~cm}^{-1}$. Uncertainties in the line lists of Shenstone [6] and Gilbert [2] were in the ranges $0.5 \mathrm{~cm}^{-1}$ to $0.9 \mathrm{~cm}^{-1}$ and $0.9 \mathrm{~cm}^{-1}$ to $3 \mathrm{~cm}^{-1}$, respectively. Since the measurements of Kalus et al. [1] provided level values with relative uncertainties as small as $0.003 \mathrm{~cm}^{-1}$, the line assignments of Benschop et al. can now be evaluated much more accurately. Their new level values could possibly be improved by using their measurements together with the new high-precision data. If such an improvement were achieved, the accuracy of the ionization limit derived by Benschop et al. from their level values could also be improved.

As mentioned by Kalus et al. [1], the listing of only small sets of newly identified lines impedes comparison of observed intensities and makes it difficult to search for Ag II lines in spectra of composite materials. In addition, the very different excitation conditions used make relative intensities reported by different authors incompatible with each other.

Kalus et al. [1] presented a good description of all energy levels derived from their measurements, including some revised level designations and eigenvector percentage compositions in the LS coupling scheme. However, the new designations of the $4 \mathrm{~d}^{9} \mathrm{nl}$ levels are hardly better than the previous ones, because the levels are highly mixed in this coupling scheme. As found by Engleman et al. [8], these levels in Pd-like spectra are much better described in $J K$ coupling.

As noted by Kalus et al. [1], several earlier papers gave theoretical and experimental transition probabilities and radiative lifetimes for Ag II. However, the current Ag II line list in the NIST Atomic Spectra Database (ASD) [9] has no transition probability values. Another source of reference data, the Handbook of Basic Atomic Spectroscopic Data [10] lists only provisional theoretical data from Biémont et al. [11] without evaluated uncertainties.

The aims of the present paper are: 1) compile a critically evaluated comprehensive list of all observed spectral lines of Ag II with intensities normalized to a common scale; 2) based on the complete line list, reoptimize the energy levels and determine precise Ritz wavelengths for all observed lines; 3) give a good theoretical description of all known energy levels in the most appropriate coupling scheme providing unambiguous level designations; 4) validate the line classifications given by Benschop et al. [5] and derive an improved value for the ionization limit; and 5) compile a set of critically evaluated transition probabilities.

\section{Observed Lines of Ag II}

Table 1 is the total list of observed lines of Ag II compiled from the measurements of Kalus et al. [1], Rasmussen [3,4], Gilbert [2], Blair [7], and Shenstone [6]. These measurements and their uncertainties are discussed in detail below. The observations of Benschop et al. [5] are also discussed. However, since their results were found to be incorrect, they were not included in the new level optimization.

The observed wavelengths and their uncertainties, as given in Table 1, are discussed below in this section. All uncertainties given in this work are meant to be on the level of one standard deviation. The observed relative intensities given in Table 1 were converted to an arbitrary uniform scale as described in Sec. 5.

The Ritz wavelengths given in Table 1 were obtained from the optimized energy levels (see Sec. 3). Both observed and Ritz wavelengths above $2000 \AA$ are given in standard air. They were converted from the vacuum wave numbers using the five-parameter formula of Peck and Reeder [12]. 
Table 1. Spectral lines of Ag II

\begin{tabular}{|c|c|c|c|c|c|c|c|c|c|c|c|c|c|}
\hline $\begin{array}{l}I_{\mathrm{obs}}^{\mathrm{a}} \\
\text { arb.u. }\end{array}$ & $\begin{array}{c}\lambda_{\mathrm{obs}}^{\mathrm{b}} \\
\AA\end{array}$ & $\begin{array}{l}\begin{array}{l}\sigma_{\mathrm{obs}} \\
\mathrm{cm}^{-1}\end{array} \\
\end{array}$ & $\begin{array}{l}\lambda_{\text {Ritz }} \\
\AA\end{array}$ & $\begin{array}{c}\Delta \lambda_{\text {obs-Ritz }} \\
\AA\end{array}$ & Lower level & & Upper level & & $\begin{array}{l}\text { Line } \\
\text { Ref. }^{c} \\
\end{array}$ & $\begin{array}{l}A \\
\mathrm{~s}^{-1} \\
\end{array}$ & Acc. ${ }^{\mathrm{d}}$ & $\begin{array}{l}\text { TP } \\
\text { Ref. }^{\text {e }}\end{array}$ & Note $^{f}$ \\
\hline 1400 & 9052.697(18) & 11043.401 & & & $4 d_{3 / 2}^{9} 6 s$ & ${ }^{2}[3 / 2]_{1}$ & $4 d_{3 / 2}^{9} 6 p$ & ${ }^{2}[1 / 2]^{\circ}{ }_{0}$ & R40bc & $4.1 \mathrm{e}+07$ & C & TW & S \\
\hline 2900 & 9001.362(18) & 11106.381 & $9001.360(11)$ & 0.002 & $4 d^{9}{ }_{5 / 2} 6 s$ & ${ }^{2}[5 / 2]_{2}$ & $4 d^{9}{ }_{5 / 2} 6 p$ & ${ }^{2}[7 / 2]^{\circ}{ }_{3}$ & R40bc & $2.9 \mathrm{e}+07$ & $\mathrm{C}$ & TW & \\
\hline 550 & 8995.174(18) & 11114.021 & 8995.181(14) & -0.006 & $4 d_{3 / 2}^{9} 6 s$ & ${ }^{2}[3 / 2]_{2}$ & $4 d^{9}{ }_{3 / 2} 6 p$ & ${ }^{2}[5 / 2]_{2}^{\circ}$ & R40bc & $1.10 \mathrm{e}+07$ & $\mathrm{C}$ & TW & \\
\hline 1300 & 8775.316(18) & 11392.472 & 8775.306(13) & 0.010 & $4 d_{3 / 2}^{9} 6 s$ & ${ }^{2}[3 / 2]_{1}$ & $4 d_{3 / 2}^{9} 6 p$ & ${ }^{2}[5 / 2]_{2}^{\circ}$ & R40bc & $3.0 \mathrm{e}+07$ & C & TW & \\
\hline 2800 & 8748.317(18) & 11427.632 & 8748.307(11) & 0.010 & $4 d_{3 / 2}^{9} 6 s$ & ${ }^{2}[3 / 2]_{2}$ & $4 d^{9}{ }_{3 / 2} 6 p$ & ${ }^{2}[1 / 2]^{\circ}{ }_{1}$ & R40bc & $4.3 e^{+07}$ & $\mathrm{C}$ & TW & \\
\hline 260 & $8725.9(23)$ & 11457 & $8726.37(7)$ & -0.5 & $4 d^{9}{ }_{5 / 2} 6 p$ & ${ }^{2}[5 / 2]^{\circ}{ }_{3}$ & $4 d^{9}{ }_{5 / 2} 7 \mathrm{~s}$ & ${ }^{2}[5 / 2]_{3}$ & G35c & $1.8 \mathrm{e}+07$ & $\mathrm{C}$ & TW & \\
\hline 1600 & 8705.390(17) & 11483.982 & $8705.390(11)$ & 0.000 & $4 d^{9}{ }_{5 / 2} 6 s$ & ${ }^{2}[5 / 2]_{3}$ & $4 d^{9}{ }_{5 / 2} 6 p$ & ${ }^{2}[7 / 2]_{3}^{\circ}$ & R40bc & $1.17 \mathrm{e}+07$ & C & TW & \\
\hline 220 & $8541.0(22)$ & 11705 & $8541.87(5)$ & -0.9 & $4 d^{9}{ }_{5 / 2} 6 p$ & ${ }^{2}[5 / 2]^{\circ}{ }_{2}$ & $4 d^{9}{ }_{5 / 2} 7 \mathrm{~s}$ & ${ }^{2}[5 / 2]_{2}$ & G35c & $2.5 e+07$ & C & TW & \\
\hline 390 & 8540.184(17) & 11706.133 & $8540.196(10)$ & -0.011 & $4 d_{3 / 2}^{9} 6 s$ & ${ }^{2}[3 / 2]_{1}$ & $4 d_{3 / 2}^{9} 6 p$ & ${ }^{2}[1 / 2]^{\circ}{ }_{1}$ & R40bc & $1.4 \mathrm{e}+06$ & $\mathrm{D}^{+}$ & TW & \\
\hline 2600 & 8492.643(17) & 11771.663 & 8492.661(17) & -0.018 & $4 d^{9}{ }_{3 / 2} 6 s$ & ${ }^{2}[3 / 2]_{2}$ & $4 d^{9}{ }_{3 / 2} 6 p$ & ${ }^{2}[5 / 2]^{\circ}{ }_{3}$ & R40bc & $4.3 \mathrm{e}+07$ & $\mathrm{C}$ & TW & \\
\hline 170 & $8457.546(17)$ & 11820.513 & 8457.536(5) & 0.010 & $4 d^{9}{ }_{3 / 2} 6 s$ & ${ }^{2}[3 / 2]_{2}$ & $4 d_{3 / 2}^{9} 6 p$ & ${ }^{2}[3 / 2]^{\circ}{ }_{1}$ & R40bc & & & & \\
\hline 270 & $8431.5(21)$ & 11857 & $8431.20(6)$ & 0.3 & $4 d^{9}{ }_{3 / 2} 6 p$ & ${ }^{2}[3 / 2]^{\circ}{ }_{1}$ & $4 d^{9}{ }_{3 / 2} 7 \mathrm{~s}$ & ${ }^{2}[3 / 2]_{1}$ & G35c & $2.4 \mathrm{e}+07$ & C & TW & \\
\hline 5000 & 8403.8338(21) & 11896.062 & $8403.8336(21)$ & 0.0001 & $4 d^{9}{ }_{5 / 2}^{6} 6 s$ & ${ }^{2}[5 / 2]_{3}$ & $4 d^{9}{ }_{5 / 2} 6 p$ & ${ }^{2}[7 / 2]^{\circ}{ }_{4}$ & K02c & $4.7 \mathrm{e}+07$ & C & TW & Las \\
\hline 2000 & 8379.578(4) & 11930.496 & 8379.578(4) & 0.000 & $4 d^{9}{ }_{5 / 2} 6 s$ & ${ }^{2}[5 / 2]_{2}$ & $4 d^{9}{ }_{5 / 2} 6 p$ & ${ }^{2}[3 / 2]^{\circ}{ }_{1}$ & K02c & $5.0 \mathrm{e}+07$ & $\mathrm{C}$ & TW & \\
\hline 260 & $8362.4(21)$ & 11955 & 8361.16(6) & 1.2 & $4 d_{3 / 2}^{9} 6 p$ & ${ }^{2}[3 / 2]^{\circ}{ }_{1}$ & $4 \mathrm{~d}_{3 / 2}^{9} 7 \mathrm{~s}$ & ${ }^{2}[3 / 2]_{2}$ & G35c & & & & \\
\hline 2100 & 8324.708(3) & 12009.132 & $8324.707(3)$ & 0.002 & $4 d^{9}{ }_{5 / 2}^{6 s}$ & ${ }^{2}[5 / 2]_{2}$ & $4 d^{9}{ }_{5 / 2} 6 p$ & ${ }^{2}[5 / 2]^{\circ}{ }_{2}$ & K02c & $4.7 \mathrm{e}+07$ & C & TW & Las \\
\hline 2100 & $8297.8(3)$ & 12048.1 & 8297.32(6) & 0.5 & $4 d^{9}{ }_{5 / 2} 6 p$ & ${ }^{2}[7 / 2]^{\circ}{ }_{4}$ & $4 d^{9}{ }_{5 / 2} 7 \mathrm{~s}$ & ${ }^{2}[5 / 2]_{3}$ & G35c & $3.4 \mathrm{e}+07$ & C & TW & \\
\hline 1100 & 8287.284(16) & 12063.364 & $8287.277(7)$ & 0.006 & $4 \mathrm{~d}_{3 / 2}^{9} 6 \mathrm{~s}$ & ${ }^{2}[3 / 2]_{2}$ & $4 d_{3 / 2}^{9} 6 p$ & ${ }^{2}[3 / 2]^{\circ}{ }_{2}$ & R40bc & $3.0 \mathrm{e}+07$ & $\mathrm{C}$ & TW & \\
\hline 950 & $8262.874(14)$ & 12099.001 & 8262.875(5) & -0.001 & $4 d_{3 / 2}^{9} 6 s$ & ${ }^{2}[3 / 2]_{1}$ & $4 d^{9}{ }_{3 / 2} 6 p$ & ${ }^{2}[3 / 2]^{\circ}{ }_{1}$ & K02c & $4.3 \mathrm{e}+07$ & $\mathrm{C}$ & TW & \\
\hline 1500 & $8254.777(4)$ & 12110.868 & $8254.7772(20)$ & 0.000 & $4 d^{9}{ }_{5 / 2} 6 s$ & ${ }^{2}[5 / 2]_{2}$ & $4 d^{9}{ }_{5 / 2} 6 p$ & ${ }^{2}[5 / 2]_{3}^{\circ}$ & K02c & $1.4 \mathrm{e}+07$ & $\mathrm{C}$ & TW & Las \\
\hline 270 & $8224.770(16)$ & 12155.054 & $8224.757(5)$ & 0.013 & $4 d^{9}{ }_{5 / 2} 6 s$ & ${ }^{2}[5 / 2]_{3}$ & $4 d^{8}\left({ }^{3} F\right) 5 s 5 p\left({ }^{3} P^{\circ}\right)$ & ${ }^{5} \mathrm{G}^{\circ}{ }_{4}$ & R40bc & $1.7 \mathrm{e}+06$ & $\mathrm{D}^{+}$ & TW & \\
\hline 810 & $8100.253(20)$ & 12341.90 & $8100.289(7)$ & -0.036 & $4 d^{9}{ }_{3 / 2} 6 s$ & ${ }^{2}[3 / 2]_{1}$ & $4 d^{9}{ }_{3 / 2} 6 p$ & ${ }^{2}[3 / 2]_{2}^{\circ}$ & R40bc & $1.3 \mathrm{e}+07$ & $\mathrm{C}$ & TW & \\
\hline 220bl & 8096.3(20) & 12348 & 8095.17(5) & 1.1 & $4 d_{3 / 2}^{9} 6 p$ & ${ }^{2}[1 / 2]^{\circ}{ }_{1}$ & $4 d_{3 / 2}^{9} 7 \mathrm{~s}$ & ${ }^{2}[3 / 2]_{2}$ & G35c & $1.8 \mathrm{e}+07$ & $\mathrm{C}$ & TW & \\
\hline 310 & 8023.0(3) & 12460.7 & 8022.93(6) & 0.1 & $4 d^{9}{ }_{5 / 2} 6 p$ & ${ }^{2}[7 / 2]^{\circ}{ }_{3}$ & $4 d^{9}{ }_{5 / 2} 7 \mathrm{~s}$ & ${ }^{2}[5 / 2]_{3}$ & G35c & $8.9 \mathrm{e}+06$ & $\mathrm{C}$ & TW & \\
\hline 2600 & 8005.1863(19) & 12488.467 & $8005.1861(15)$ & 0.0001 & $4 d^{9}{ }_{5 / 2} 6 s$ & ${ }^{2}[5 / 2]_{3}$ & $4 d^{9}{ }_{5 / 2} 6 p$ & ${ }^{2}[5 / 2]^{\circ}{ }_{3}$ & K02c & $3.8 \mathrm{e}+07$ & C & TW & Las \\
\hline 420 & 7957.3(3) & 12563.6 & $7957.08(5)$ & 0.2 & $4 d_{3 / 2}^{9} 6 p$ & ${ }^{2}[5 / 2]^{\circ}{ }_{2}$ & $4 \mathrm{~d}_{3 / 2}^{9} 7 \mathrm{~s}$ & ${ }^{2}[3 / 2]_{1}$ & G35c & $3.7 \mathrm{e}+07$ & $\mathrm{C}$ & TW & \\
\hline 1800 & $7930.2(3)$ & 12606.5 & 7930.19(4) & 0.0 & $4 d^{9}{ }_{5 / 2} 6 p$ & ${ }^{2}[7 / 2]_{3}^{\circ}$ & $4 d^{9}{ }_{5 / 2} 7 \mathrm{~s}$ & ${ }^{2}[5 / 2]_{2}$ & G35c & $3.2 \mathrm{e}+07$ & C & TW & \\
\hline 44 & 7439.5(17) & 13438 & $7442.896(4)$ & -3.4 & $4 d^{9}{ }_{5 / 2} 6 s$ & ${ }^{2}[5 / 2]_{2}$ & $4 d^{8}\left({ }^{3} \mathrm{~F}\right) 5 \mathrm{~s} 5 \mathrm{p}\left({ }^{3} \mathrm{P}^{\circ}\right)$ & ${ }^{5} \mathrm{G}^{\circ}{ }_{3}$ & G35c & & & & \\
\hline
\end{tabular}


Volume 118 (2013) http://dx.doi.org/10.6028/jres.118.009

Journal of Research of the National Institute of Standards and Technology

\begin{tabular}{|c|c|c|c|c|c|c|c|c|c|c|c|c|c|}
\hline $\begin{array}{c}I_{\text {obs }}{ }^{\mathrm{a}} \\
\text { arb.u. }\end{array}$ & $\begin{array}{c}\lambda_{\text {obs }} \mathrm{b} \\
\AA \\
\AA\end{array}$ & $\begin{array}{c}\begin{array}{c}\sigma_{\mathrm{obs}} \\
\mathrm{cm}^{-1}\end{array} \\
\end{array}$ & $\begin{array}{c}\lambda_{\text {Ritz }} \\
\AA \\
\end{array}$ & $\begin{array}{c}\Delta \lambda_{\text {obs-Ritz }} \\
\AA\end{array}$ & Lower level & & Upper level & & $\begin{array}{l}\text { Line } \\
\text { Ref. }^{c}\end{array}$ & $\begin{array}{c}A \\
\mathrm{~s}^{-1}\end{array}$ & Acc. ${ }^{\mathrm{d}}$ & $\begin{array}{l}\text { TP } \\
\text { Ref. }^{\text {e }}\end{array}$ & Note $^{f}$ \\
\hline 250 & 7239.376(16) & 13809.54 & 7239.381(4) & -0.006 & $4 d^{9}{ }_{5 / 2} 6 s$ & ${ }^{2}[5 / 2]_{3}$ & $4 d^{8}\left({ }^{3} F\right) 5 s 5 p\left({ }^{3} P^{\circ}\right)$ & ${ }^{5} \mathrm{G}_{3}^{\circ}$ & R40bc & & & & \\
\hline $77 \mathrm{~d}$ & 7198.5(16) & 13888 & $7198.4980(21)$ & 0.0 & $4 d^{9}{ }_{5 / 2}^{6} 6 s$ & ${ }^{2}[5 / 2]_{2}$ & $4 d^{8}\left({ }^{3} F\right) 5 s 5 p\left({ }^{3} P^{\circ}\right)$ & ${ }^{5} \mathrm{D}^{\circ}{ }_{1}$ & G35c & & & & \\
\hline 200 & 6998.91(20) & 14284.0 & $6999.06(4)$ & -0.15 & $4 d^{8}\left({ }^{3} F\right) 5 s 5 p\left({ }^{3} P^{\circ}\right)$ & ${ }^{5} \mathrm{D}^{\circ}{ }_{1}$ & $4 d^{9}{ }_{3 / 2} 7 \mathrm{~s}$ & ${ }^{2}[3 / 2]_{1}$ & G35c & & & & \\
\hline 68 & 6939.988(10) & 14405.274 & 6939.987(3) & 0.000 & $4 d^{9} 5$ p & ${ }^{2}[5 / 2]_{2}^{\circ}$ & $4 d^{8} 5 s^{2}$ & ${ }^{3} \mathrm{~F}_{2}$ & K02c & & & & \\
\hline 69bl & 6893.7(14) & 14502 & 6892.692(4) & 1.0 & $4 d^{9}{ }_{5 / 2}^{6} 6 s$ & ${ }^{2}[5 / 2]_{2}$ & $4 d^{8}\left({ }^{3} F\right) 5 s 5 p\left({ }^{3} P^{\circ}\right)$ & ${ }^{5} \mathrm{G}_{2}^{\circ}$ & G35c & & & & \\
\hline 130 & 6717.36(18) & 14882.7 & $6717.800(4)$ & -0.44 & $4 d^{9}{ }_{5 / 2}^{6} 6 s$ & ${ }^{2}[5 / 2]_{3}$ & $4 d^{8}\left({ }^{3} F\right) 5 s 5 p\left({ }^{3} P^{\circ}\right)$ & ${ }^{5} \mathrm{G}_{2}^{\circ}$ & G35c & & & & \\
\hline 81 & 6697.223(13) & 14927.44 & $6697.2424(9)$ & -0.019 & $4 d^{9}{ }_{3 / 2} 5 p$ & ${ }^{2}[1 / 2]^{\circ}{ }_{1}$ & $4 d^{8} 5 s^{2}$ & ${ }^{3} \mathrm{P}_{2}$ & R40bc & & & & \\
\hline 64 & 6505.747(13) & 15366.78 & $6505.7672(7)$ & -0.021 & $4 d^{9}{ }_{3 / 2} 5 p$ & ${ }^{2}[3 / 2]^{\circ}{ }_{1}$ & $4 d^{8} 5 s^{2}$ & ${ }^{3} \mathrm{P}_{2}$ & R40bc & & & & \\
\hline 60 & 6403.6(12) & 15612 & $6405.327(7)$ & -1.8 & $4 d^{9}{ }_{5 / 2} 6 s$ & ${ }^{2}[5 / 2]_{2}$ & $4 d^{9}{ }_{3 / 2} 6 p$ & ${ }^{2}[5 / 2]_{2}^{\circ}$ & G35c & & & & Las \\
\hline 67 & $6331.275(12)$ & 15790.24 & $6331.2484(24)$ & 0.027 & $4 d^{9}{ }_{5 / 2} 5 p$ & ${ }^{2}[7 / 2]_{3}^{\circ}$ & $4 d^{8} 5 s^{2}$ & ${ }^{3} \mathrm{~F}_{3}$ & R40bc & & & & \\
\hline 110 & $6279.154(12)$ & 15921.31 & 6279.149(6) & 0.005 & $4 d^{9}{ }_{5 / 2} 6 s$ & ${ }^{2}[5 / 2]_{2}$ & $4 d_{3 / 2}^{9} 6 p$ & ${ }^{2}[1 / 2]_{1}^{\circ}$ & R40bc & & & & \\
\hline 38 & 6255.896(12) & 15980.50 & $6255.8897(24)$ & 0.006 & $4 d^{9}{ }_{5 / 2} 5 p$ & ${ }^{2}[3 / 2]_{1}^{\circ}$ & $4 d^{8} 5 s^{2}$ & ${ }^{3} \mathrm{~F}_{2}$ & R40bc & & & & \\
\hline 36 & $5734.226(3)$ & 17434.308 & $5734.2293(20)$ & -0.003 & $4 d^{9}{ }_{5 / 2} 5 p$ & ${ }^{2}[7 / 2]_{3}^{\circ}$ & $4 d^{8} 5 s^{2}$ & ${ }^{3} \mathrm{~F}_{2}$ & K02c & & & & \\
\hline 21000 & $5622.4822(9)$ & 17780.803 & $5622.4820(10)$ & 0.0003 & $4 d^{9}{ }_{5 / 2} 5 d$ & ${ }^{2}[7 / 2]_{4}$ & $4 d^{9}{ }_{5 / 2} 4 f$ & ${ }^{2}[9 / 2]_{5}^{\circ}$ & K02c & $1.4 \mathrm{e}+08$ & $\mathrm{C}$ & TW & \\
\hline 43 & $5620.974(3)$ & 17785.574 & $5620.9724(19)$ & 0.002 & $4 d^{9} 5 / 25$ & ${ }^{2}[3 / 2]^{\circ}{ }_{2}$ & $4 d^{8} 5 s^{2}$ & ${ }^{3} \mathrm{~F}_{3}$ & K02c & & & & \\
\hline $5400 *$ & $5610.6066(16)$ & 17818.438 & 5610.6081(19) & -0.001 & $4 d^{9}{ }_{5 / 2} 5 d$ & ${ }^{2}[7 / 2]_{3}$ & $4 d^{9}{ }_{5 / 2} 4 f$ & ${ }^{2}[5 / 2]_{2}^{\circ}$ & K02c & & & & \\
\hline $5400 *$ & $5610.6066(16)$ & 17818.438 & $5610.6070(10)$ & -0.0004 & $4 d^{9}{ }_{5 / 2} 5 d$ & ${ }^{2}[7 / 2]_{4}$ & $4 d^{9}{ }_{5 / 2} 4 f$ & ${ }^{2}[7 / 2]^{\circ}{ }_{4}$ & K02c & $4.0 \mathrm{e}+07$ & $\mathrm{C}$ & TW & \\
\hline 4200 & $5589.7828(16)$ & 17884.817 & $5589.7829(9)$ & 0.0000 & $4 d^{9}{ }_{5 / 2} 5 d$ & ${ }^{2}[5 / 2]_{2}$ & $4 d^{9}{ }_{5 / 2} 4 f$ & ${ }^{2}[7 / 2]^{\circ}{ }_{3}$ & K02c & $6.9 \mathrm{e}+07$ & C & TW & \\
\hline 1700 & $5588.417(3)$ & 17889.189 & $5588.4183(9)$ & -0.002 & $4 d^{9}{ }_{5 / 2} 5 d$ & ${ }^{2}[7 / 2]_{4}$ & $4 d^{9}{ }_{5 / 2} 4 f$ & ${ }^{2}[9 / 2]^{\circ}{ }_{4}$ & K02c & $1.6 \mathrm{e}+07$ & C & TW & \\
\hline 2400 & $5579.6777(22)$ & 17917.207 & $5579.6782(9)$ & -0.0005 & $4 d^{9} 5 / 2 \mathrm{~d}$ & ${ }^{2}[7 / 2]_{3}$ & $4 d^{9}{ }_{5 / 2} 4 f$ & ${ }^{2}[7 / 2]^{\circ}{ }_{3}$ & K02c & $3.7 \mathrm{e}+07$ & C & TW & \\
\hline 2300 & $5573.8261(22)$ & 17936.017 & $5573.8257(9)$ & 0.0004 & $4 d_{5 / 2}^{9} 5 d$ & ${ }^{2}[7 / 2]_{3}$ & $4 d^{9}{ }_{5 / 2} 4 f$ & ${ }^{2}[7 / 2]^{\circ}{ }_{4}$ & K02c & $3.2 \mathrm{e}+07$ & C & TW & \\
\hline 2300 & $5558.1401(22)$ & 17986.635 & $5558.1412(12)$ & -0.0011 & $4 d^{9}{ }_{5 / 2}^{5 d}$ & ${ }^{2}[5 / 2]_{3}$ & $4 d_{5 / 2}^{9} 4 \mathrm{f}$ & ${ }^{2}[3 / 2]^{\circ}{ }_{2}$ & K02c & $1.9 \mathrm{e}+07$ & C & TW & \\
\hline 12000 & $5551.9265(9)$ & 18006.765 & $5551.9264(9)$ & 0.0001 & $4 d^{9}{ }_{5 / 2}^{5 d}$ & ${ }^{2}[7 / 2]_{3}$ & $4 d^{9}{ }_{5 / 2} 4 f$ & ${ }^{2}[9 / 2]^{\circ}{ }_{4}$ & K02c & $1.07 \mathrm{e}+08$ & C & TW & \\
\hline 2700 & 5543.2124(18) & 18035.072 & $5543.2121(18)$ & 0.0003 & $4 d^{9}{ }_{5 / 2} 5 d$ & ${ }^{2}[5 / 2]_{2}$ & $4 d^{8}\left({ }^{3} F\right) 5 s 5 p\left({ }^{3} \mathrm{P}^{\circ}\right)$ & ${ }^{1} \mathrm{~F}_{3}^{\circ}$ & K02c & $2.1 \mathrm{e}+07$ & C & TW & \\
\hline 27 & $5538.4490(21)$ & 18050.583 & $5538.4481(6)$ & 0.0009 & $4 d_{3 / 2}^{9} 5 p$ & ${ }^{2}[3 / 2]^{\circ}{ }_{2}$ & $4 d^{8} 5 s^{2}$ & ${ }^{3} \mathrm{P}_{1}$ & K02c & & & & \\
\hline 2300 & 5493.8309(18) & 18197.179 & 5493.8302(9) & 0.0007 & $4 d^{9}{ }_{5 / 2} 5 d$ & ${ }^{2}[5 / 2]_{3}$ & $4 d_{5 / 2}^{9} 4 f$ & ${ }^{2}[7 / 2]^{\circ}{ }_{3}$ & K02c & $2.8 \mathrm{e}+07$ & C & TW & \\
\hline 8300 & $5488.1567(9)$ & 18215.993 & 5488.1562(7) & 0.0004 & $4 d^{9}{ }_{5 / 2} 5 d$ & ${ }^{2}[5 / 2]_{3}$ & $4 d^{9}{ }_{5 / 2} 4 f$ & ${ }^{2}[7 / 2]^{\circ}{ }_{4}$ & K02c & $7.8 \mathrm{e}+07$ & C & TW & \\
\hline 1500 & $5478.6597(21)$ & 18247.569 & 5478.6589(12) & 0.0009 & $4 d^{9}{ }_{5 / 2} 5 d$ & ${ }^{2}[3 / 2]_{2}$ & $4 d^{9}{ }_{5 / 2} 4 f$ & ${ }^{2}[1 / 2]^{\circ}{ }_{1}$ & K02c & $5.4 \mathrm{e}+07$ & C & TW & \\
\hline 50 & 5440.9099(9) & 18374.172 & $5440.9104(5)$ & -0.0006 & $4 d^{9}{ }_{3 / 2} 5 p$ & ${ }^{2}[5 / 2]_{2}^{\circ}$ & $4 d^{8} 5 s^{2}$ & ${ }^{3} \mathrm{P}_{2}$ & K02c & & & & \\
\hline 2200 & $5424.0511(15)$ & 18431.281 & $5424.0509(12)$ & 0.0003 & $4 d^{9}{ }_{5 / 2} 5 d$ & ${ }^{2}[3 / 2]_{2}$ & $4 d_{5 / 2}^{9} 4 \mathrm{f}$ & ${ }^{2}[3 / 2]^{\circ}{ }_{2}$ & K02c & $7.4 \mathrm{e}+07$ & C & TW & \\
\hline
\end{tabular}


Volume 118 (2013) http://dx.doi.org/10.6028/jres.118.009

Journal of Research of the National Institute of Standards and Technology

\begin{tabular}{|c|c|c|c|c|c|c|c|c|c|c|c|c|c|}
\hline $\begin{array}{l}I_{\text {obs }}{ }^{\mathrm{a}} \\
\text { arb.u. }\end{array}$ & $\begin{array}{c}\lambda_{\text {obs }}{ }^{b} \\
\AA\end{array}$ & $\begin{array}{c}\begin{array}{c}\sigma_{\mathrm{obs}} \\
\mathrm{cm}^{-1}\end{array} \\
\mathrm{c}\end{array}$ & $\stackrel{\lambda_{\text {Ritz }}}{\AA}$ & $\begin{array}{c}\Delta \lambda_{\text {obs-Ritz }} \\
\AA\end{array}$ & Lower level & & Upper level & & $\begin{array}{l}\text { Line } \\
\text { Ref. }^{c}\end{array}$ & $\begin{array}{l}A \\
\mathrm{~s}^{-1}\end{array}$ & Acc. ${ }^{\mathrm{d}}$ & $\begin{array}{l}\text { TP } \\
\text { Ref. }^{\text {e }}\end{array}$ & Note $^{f}$ \\
\hline 740 & 5411.936(3) & 18472.540 & 5411.9338(15) & 0.002 & $4 d^{9}{ }_{5 / 2} 5 d$ & ${ }^{2}[3 / 2]_{1}$ & $4 d_{5 / 2}^{9} 4 \mathrm{f}$ & ${ }^{2}[3 / 2]^{\circ}{ }_{1}$ & K02c & $8.9 \mathrm{e}+07$ & C & TW & \\
\hline 1800 & $5410.8114(15)$ & 18476.380 & $5410.8117(15)$ & -0.0003 & $4 d^{9}{ }_{5 / 2} 5 d$ & ${ }^{2}[3 / 2]_{2}$ & $4 d^{9} 5 / 24 f$ & ${ }^{2}[3 / 2]^{\circ}{ }_{1}$ & K02c & $3.6 \mathrm{e}+06$ & $\mathrm{D}+$ & TW & \\
\hline 15000 & 5403.1323(9) & 18502.639 & & & $4 d^{9} 5 / 2 \mathrm{~d}$ & ${ }^{2}[9 / 2]_{4}$ & $4 d^{9} 5 / 24 f$ & ${ }^{2}[11 / 2]^{\circ}{ }_{5}$ & K02c & $1.8 \mathrm{e}+08$ & $\mathrm{C}$ & TW & S \\
\hline 20000 & $5400.1037(9)$ & 18513.016 & & & $4 d^{9}{ }_{5 / 2} 5 d$ & ${ }^{2}[9 / 2]_{5}$ & $4 d^{9}{ }_{5 / 2} 4 \mathrm{f}$ & ${ }^{2}[11 / 2]^{\circ}{ }_{6}$ & K02c & $1.8 \mathrm{e}+08$ & C & TW & $\mathrm{S}$ \\
\hline 1500 & 5392.4686(17) & 18539.228 & 5392.4682(17) & 0.0004 & $4 d^{9}{ }_{5 / 2} 5 d$ & ${ }^{2}[3 / 2]_{1}$ & $4 d^{9} 5 / 24 f$ & ${ }^{2}[5 / 2]_{2}^{\circ}$ & K02c & $7.9 \mathrm{e}+07$ & C & TW & \\
\hline 38 & 5373.8212(12) & 18603.559 & 5373.8219(5) & -0.0007 & $4 d^{9}{ }_{3 / 2}^{5} p$ & ${ }^{2}[1 / 2]^{\circ}{ }_{1}$ & $4 d^{8} 5 s^{2}$ & ${ }^{3} \mathrm{P}_{1}$ & K02c & & & & \\
\hline 1600 & $5362.7877(14)$ & 18641.834 & 5362.7883(9) & -0.0006 & $4 d^{9}{ }_{5 / 2}^{5} \mathrm{~d}$ & ${ }^{2}[3 / 2]_{2}$ & $4 d^{9} 5 / 24 f$ & ${ }^{2}[7 / 2]^{\circ}{ }_{3}$ & K02c & $2.2 \mathrm{e}+07$ & C & TW & \\
\hline 2900 & $5340.0267(11)$ & 18721.291 & $5340.0267(9)$ & 0.0000 & $4 d^{9}{ }_{5 / 2} 5 d$ & ${ }^{2}[9 / 2]_{5}$ & $4 d^{9} 5 / 24$ & ${ }^{2}[9 / 2]^{\circ}{ }_{5}$ & K02c & $3.4 \mathrm{e}+07$ & C & TW & \\
\hline 1300 & $5332.5043(14)$ & 18747.700 & 5332.5049(9) & -0.0006 & $4 d^{9}{ }_{5 / 2} 5 d$ & ${ }^{2}[9 / 2]_{4}$ & $4 d^{9} / 24 f$ & ${ }^{2}[7 / 2]^{\circ}{ }_{4}$ & K02c & $2.0 \mathrm{e}+07$ & C & TW & \\
\hline 21 & $5319.7018(20)$ & 18792.818 & 5319.7015(9) & 0.0004 & $4 d_{3 / 2}^{9} 5 p$ & ${ }^{2}[1 / 2]^{\circ}{ }_{1}$ & $4 d^{8} 5 s^{2}$ & ${ }^{3} \mathrm{P}_{0}$ & K02c & & & & \\
\hline 48 & 5317.2122(8) & 18801.617 & $5317.2120(5)$ & 0.0003 & $4 d^{9}{ }_{5 / 2} 5 p$ & ${ }^{2}[5 / 2]^{\circ}{ }_{3}$ & $4 d^{8} 5 s^{2}$ & ${ }^{3} \mathrm{P}_{2}$ & K02c & & & & \\
\hline 1200 & $5312.4571(17)$ & 18818.446 & 5312.4574(8) & -0.0003 & $4 d^{9}{ }_{5 / 2} 5 d$ & ${ }^{2}[9 / 2]_{4}$ & $4 d_{5 / 2}^{9} 4 \mathrm{f}$ & ${ }^{2}[9 / 2]^{\circ}{ }_{4}$ & K02c & $1.6 \mathrm{e}+07$ & $\mathrm{C}$ & TW & \\
\hline 27 & 5198.171(8) & 19232.18 & 5198.1783(8) & -0.007 & $4 d^{9}{ }_{3 / 2} 5 p$ & ${ }^{2}[3 / 2]^{\circ}{ }_{1}$ & $4 d^{8} 5 s^{2}$ & ${ }^{3} \mathrm{P}_{0}$ & $\mathrm{R} 40 \mathrm{ac}$ & & & & \\
\hline 1700 & $5142.8156(11)$ & 19439.186 & $5142.8157(11)$ & -0.0002 & $4 d^{9}{ }_{5 / 2} 5 d$ & ${ }^{2}[1 / 2]_{1}$ & $4 d_{5 / 2}^{9} 4 \mathrm{f}$ & ${ }^{2}[1 / 2]^{\circ}{ }_{1}$ & $\mathrm{~K} 02 \mathrm{c}$ & $1.3 \mathrm{e}+08$ & $\mathrm{C}$ & TW & \\
\hline 720 & $5137.2470(16)$ & 19460.257 & $5137.2469(16)$ & 0.0001 & $4 d^{9} 5 / 2 \mathrm{~d}$ & ${ }^{2}[1 / 2]_{1}$ & $4 d^{8}\left({ }^{3} \mathrm{~F}\right) 5 \mathrm{~s} 5 \mathrm{p}\left({ }^{3} \mathrm{P}^{\circ}\right)$ & ${ }^{1} \mathrm{D}_{2}^{\circ}$ & K02c & $1.8 \mathrm{e}+07$ & C & TW & \\
\hline 160 & $5027.3430(8)$ & 19885.677 & 5027.3432(3) & -0.0002 & $4 d_{3 / 2}^{9} 5 p$ & ${ }^{2}[3 / 2]^{\circ}{ }_{2}$ & $4 d^{8} 5 s^{2}$ & ${ }^{1} \mathrm{D}_{2}$ & K02c & $3.4 \mathrm{e}+05$ & C+ & $\mathrm{C} 05$ & Las \\
\hline 17 & 4983.2786(12) & 20061.513 & 4983.2786(4) & 0.0000 & $4 d^{9}{ }_{5 / 2} 5 p$ & ${ }^{2}[5 / 2]_{2}^{\circ}$ & $4 d^{8} 5 s^{2}$ & ${ }^{3} \mathrm{P}_{2}$ & K02c & & & & \\
\hline 18 & 4891.335(10) & 20438.61 & 4891.3259(4) & 0.009 & $4 d^{9}{ }_{3 / 2}^{5} p$ & ${ }^{2}[1 / 2]^{\circ}{ }_{1}$ & $4 d^{8} 5 s^{2}$ & ${ }^{1} \mathrm{D}_{2}$ & R40ac & $1.00 \mathrm{e}+05$ & B & $\mathrm{C} 05$ & \\
\hline 260 & 4788.3966(7) & 20877.981 & 4788.3966(3) & 0.0000 & $4 d^{9}{ }_{3 / 2}^{5} p$ & ${ }^{2}[3 / 2]^{\circ}{ }_{1}$ & $4 d^{8} 5 s^{2}$ & ${ }^{1} \mathrm{D}_{2}$ & K02c & $9.5 e^{+}+05$ & C+ & $\mathrm{C} 05$ & Las \\
\hline 56 & 4705.873(9) & 21244.10 & 4705.879(3) & -0.007 & $4 d^{8} 5 s^{2}$ & ${ }^{1} \mathrm{D}_{2}$ & $4 d^{9}{ }_{5 / 2}^{6} 6 p$ & ${ }^{2}[7 / 2]^{\circ}{ }_{3}$ & R40bc & & & & \\
\hline 85 & $4620.4712(6)$ & 21636.754 & $4620.4711(3)$ & 0.0001 & $4 d^{9}{ }_{5 / 2}^{5 p}$ & ${ }^{2}[3 / 2]^{\circ}{ }_{1}$ & $4 d^{8} 5 s^{2}$ & ${ }^{3} \mathrm{P}_{2}$ & K02c & & & & \\
\hline 170 & $4620.0358(6)$ & 21638.793 & $4620.0355(3)$ & 0.0003 & $4 d_{3 / 2}^{9} 5 p$ & ${ }^{2}[5 / 2]^{\circ}{ }_{3}$ & $4 d^{8} 5 s^{2}$ & ${ }^{1} \mathrm{D}_{2}$ & K02c & $2.1 \mathrm{e}+05$ & $\mathrm{C}$ & $\mathrm{C} 05$ & \\
\hline 23 & 4533.812(8) & 22050.31 & 4533.8084(4) & 0.004 & $4 d_{3 / 2}^{9} 5 p$ & ${ }^{2}[5 / 2]_{2}^{\circ}$ & $4 d^{8} 5 s^{2}$ & ${ }^{3} \mathrm{P}_{1}$ & R40ac & & & & \\
\hline 62 & $4530.145(8)$ & 22068.16 & $4530.1405(10)$ & 0.005 & $4 d^{8} 5 s^{2}$ & ${ }^{1} \mathrm{D}_{2}$ & $4 d^{9}{ }_{5 / 2} 6 p$ & ${ }^{2}[3 / 2]^{\circ}{ }_{1}$ & R40bc & & & & \\
\hline 410 & 4530.39(4) & 22066.99 & $4530.41(4)$ & -0.02 & $4 d^{9}{ }_{5 / 2} 6 p$ & ${ }^{2}[5 / 2]^{\circ}{ }_{3}$ & $4 d^{9}{ }_{5 / 2} 8 s$ & ${ }^{2}[5 / 2]_{3}$ & G35c & $7.4 \mathrm{e}+06$ & $\mathrm{D}^{+}$ & TW & \\
\hline 210 & $4515.52(4)$ & 22139.65 & $4515.58(4)$ & -0.06 & $4 d^{9}{ }_{5 / 2} 6 p$ & ${ }^{2}[5 / 2]^{\circ}{ }_{3}$ & $4 d^{9}{ }_{5 / 2} 8 s$ & ${ }^{2}[5 / 2]_{2}$ & G35c & $4.1 \mathrm{e}^{+}+06$ & $\mathrm{D}^{+}$ & TW & \\
\hline 84 & 4514.053(8) & 22146.83 & 4514.0548(8) & -0.002 & $4 d^{8} 5 s^{2}$ & ${ }^{1} \mathrm{D}_{2}$ & $4 d^{9}{ }_{5 / 2} 6 p$ & ${ }^{2}[5 / 2]^{\circ}{ }_{2}$ & R40bc & & & & \\
\hline 410 & 4494.94(4) & 22241.02 & 4494.92(4) & 0.01 & $4 d^{9}{ }_{5 / 2}^{6 p}$ & ${ }^{2}[5 / 2]_{2}^{\circ}$ & $4 d^{9}{ }_{5 / 2} 8 \mathrm{~s}$ & ${ }^{2}[5 / 2]_{2}$ & G35c & $1.1 \mathrm{e}^{+} 07$ & $\mathrm{D}^{+}$ & TW & \\
\hline 110 & $4493.424(8)$ & 22248.50 & $4493.4135(6)$ & 0.011 & $4 d^{8} 5 s^{2}$ & & $4 d^{9}{ }_{5 / 2} 6 p$ & ${ }^{2}[5 / 2]^{\circ}{ }_{3}$ & R40bc & & & & \\
\hline 290d & $4488.58(20)$ & 22272.5 & 4488.66(5) & -0.08 & $4 d_{3 / 2}^{9} 6 p$ & ${ }^{2}[3 / 2]^{\circ}{ }_{2}$ & $4 d^{9}{ }_{3 / 2}^{8 s}$ & ${ }^{2}[3 / 2]_{2}$ & G35c & $6.8 \mathrm{e}+06$ & $\mathrm{D}^{+}$ & TW & \\
\hline
\end{tabular}


Volume 118 (2013) http://dx.doi.org/10.6028/jres.118.009

Journal of Research of the National Institute of Standards and Technology

\begin{tabular}{|c|c|c|c|c|c|c|c|c|c|c|c|c|c|}
\hline $\begin{array}{c}I_{\mathrm{obs}}^{\mathrm{a}} \\
\text { arb.u. }\end{array}$ & $\begin{array}{c}\lambda_{\mathrm{obs}}^{\mathrm{b}} \\
\AA \\
\end{array}$ & $\begin{array}{c}\begin{array}{c}\sigma_{\mathrm{obs}} \\
\mathrm{cm}^{-1}\end{array} \\
\end{array}$ & $\begin{array}{c}\lambda_{\text {Ritz }} \\
\AA\end{array}$ & $\begin{array}{c}\Delta \lambda_{\text {obs-Ritz }} \\
\AA\end{array}$ & Lower leve & & Upper level & & $\begin{array}{l}\text { Line } \\
\text { Ref. }^{c}\end{array}$ & $\begin{array}{c}A \\
\mathrm{~s}^{-1} \\
\end{array}$ & Acc. ${ }^{\mathrm{d}}$ & $\begin{array}{l}\text { TP } \\
\text { Ref. }^{\text {e }}\end{array}$ & Note $^{f}$ \\
\hline 210 & 4479.12(4) & 22319.57 & 4479.09(4) & 0.03 & $4 d^{9}{ }_{5 / 2} 6 p$ & ${ }^{2}[3 / 2]^{\circ}{ }_{1}$ & $4 d^{9} 5 / 28 \mathrm{~s}$ & ${ }^{2}[5 / 2]_{2}$ & G35c & $6.4 \mathrm{e}+06$ & $\mathrm{D}^{+}$ & TW & \\
\hline 290 & 4449.19(4) & 22469.67 & $4449.17(4)$ & 0.03 & $4 d_{3 / 2}^{9} 6 p$ & ${ }^{2}[3 / 2]^{\circ}{ }_{1}$ & $4 d_{3 / 2}^{9} 8 \mathrm{~s}$ & ${ }^{2}[3 / 2]_{1}$ & G35c & $1.0 \mathrm{e}+07$ & $\mathrm{D}^{+}$ & TW & \\
\hline 580 & $4430.57(4)$ & 22564.14 & $4430.63(4)$ & -0.06 & $4 d_{3 / 2}^{9} 6 p$ & ${ }^{2}[5 / 2]^{\circ}{ }_{3}$ & $4 d^{9}{ }_{3 / 2} 8 \mathrm{~s}$ & ${ }^{2}[3 / 2]_{2}$ & G35c & $1.4 \mathrm{e}+07$ & $\mathrm{D}^{+}$ & TW & \\
\hline 830 & $4412.00(4)$ & 22659.10 & $4411.96(4)$ & 0.04 & $4 d^{9}{ }_{5 / 2}^{6} 6 p$ & ${ }^{2}[7 / 2]^{\circ}{ }_{4}$ & $4 d^{9} 5 / 28 \mathrm{~s}$ & ${ }^{2}[5 / 2]_{3}$ & G35c & $1.4 \mathrm{e}+07$ & $\mathrm{D}^{+}$ & TW & \\
\hline 79 & $4385.0519(6)$ & 22798.342 & $4385.0517(4)$ & 0.0002 & $4 d_{3 / 2}^{9} 5 p$ & ${ }^{2}[1 / 2]_{0}^{\circ}$ & $4 d^{8} 5 s^{2}$ & ${ }^{3} \mathrm{P}_{1}$ & K02c & & & & \\
\hline 290 & $4364.16(4)$ & 22907.47 & $4364.09(4)$ & 0.07 & $4 d_{3 / 2}^{9} 6 p$ & ${ }^{2}[1 / 2]^{\circ}$ & $4 d_{3 / 2}^{9} 8 \mathrm{~s}$ & ${ }^{2}[3 / 2]_{2}$ & G35c & $6.6 \mathrm{e}+06$ & $\mathrm{D}^{+}$ & TW & \\
\hline 210 & 4333.14(4) & 23071.47 & 4333.16(4) & -0.02 & $4 d^{9}{ }_{5 / 2} 6 p$ & ${ }^{2}[7 / 2]^{\circ}{ }_{3}$ & $4 d^{9}{ }_{5 / 2} 8 \mathrm{~s}$ & ${ }^{2}[5 / 2]_{3}$ & G35c & $3.3 e+06$ & $\mathrm{D}^{+}$ & TW & \\
\hline 630 & 4319.59(4) & 23143.82 & 4319.59(4) & 0.00 & $4 d^{9}{ }_{5 / 2} 6 p$ & ${ }^{2}[7 / 2]^{\circ}{ }_{3}$ & $4 d^{9}{ }_{5 / 2} 8 \mathrm{~s}$ & ${ }^{2}[5 / 2]_{2}$ & G35c & $1.2 \mathrm{e}+07$ & $\mathrm{D}^{+}$ & TW & \\
\hline 290 & 4313.51(4) & 23176.44 & $4313.54(4)$ & -0.02 & $4 d_{3 / 2}^{9} 6 p$ & ${ }^{2}[5 / 2]^{\circ}{ }_{2}$ & $4 d_{3 / 2}^{9} 8 \mathrm{~s}$ & ${ }^{2}[3 / 2]_{1}$ & G35c & $1.4 \mathrm{e}+07$ & $\mathrm{D}^{+}$ & TW & \\
\hline 51 & $4241.574(9)$ & 23569.52 & $4241.5562(13)$ & 0.017 & $4 d^{8} 5 s^{2}$ & ${ }^{1} \mathrm{D}_{2}$ & $4 d^{8}\left({ }^{3} F\right) 5 s 5 p\left({ }^{3} P^{\circ}\right)$ & ${ }^{5} \mathrm{G}^{\circ}{ }_{3}$ & R40bc & & & & \\
\hline 83 & $4240.685(9)$ & 23574.46 & $4240.698(4)$ & -0.014 & $4 d^{8} 5 s^{2}$ & ${ }^{1} \mathrm{G}_{4}$ & $4 d^{9}{ }_{3 / 2} 6 p$ & ${ }^{2}[5 / 2]^{\circ}{ }_{3}$ & R40bc & & & & \\
\hline 75 & 4215.734(9) & 23713.98 & $4215.7290(11)$ & 0.005 & $4 d^{8} 5 s^{2}$ & ${ }^{3} \mathrm{P}_{0}$ & $4 d^{9}{ }_{5 / 2} 6 p$ & ${ }^{2}[3 / 2]^{\circ}{ }_{1}$ & $\mathrm{R} 40 \mathrm{bc}$ & & & & \\
\hline 34 & 4211.5277(9) & 23737.666 & $4211.5267(3)$ & 0.0010 & $4 d^{9}{ }_{5 / 2} 5 p$ & ${ }^{2}[5 / 2]_{2}^{\circ}$ & $4 d^{8} 5 s^{2}$ & ${ }^{3} \mathrm{P}_{1}$ & K02c & & & & \\
\hline 250 & $4185.4749(5)$ & 23885.420 & $4185.47499(23)$ & -0.0001 & $4 d_{3 / 2}^{9} 5 p$ & ${ }^{2}[5 / 2]^{\circ}{ }_{2}$ & $4 d^{8} 5 s^{2}$ & ${ }^{1} \mathrm{D}_{2}$ & K02c & $3.7 e+05$ & $\mathrm{C}$ & $\mathrm{C} 05$ & \\
\hline 53 & $4111.8880(5)$ & 24312.868 & $4111.88813(22)$ & -0.0001 & $4 d^{9}{ }_{5 / 2} 5 p$ & ${ }^{2}[5 / 2]^{\circ}{ }_{3}$ & $4 d^{8} 5 s^{2}$ & ${ }^{1} \mathrm{D}_{2}$ & K02c & $1.20 \mathrm{e}+05$ & B & $\mathrm{C} 05$ & \\
\hline 470 & 4085.9155(5) & 24467.412 & 4085.9155(3) & 0.0001 & $4 d_{3 / 2}^{9} 5 p$ & ${ }^{2}[5 / 2]^{\circ}{ }_{3}$ & $4 d^{8} 5 s^{2}$ & ${ }^{1} \mathrm{G}_{4}$ & K02c & $1.12 \mathrm{e}+06$ & $\mathrm{~B}+$ & $\mathrm{C} 05$ & Las \\
\hline 220 & $3985.1904(5)$ & 25085.809 & $3985.1904(3)$ & 0.0000 & $4 d_{5 / 2}^{9} 5 p$ & ${ }^{2}[3 / 2]_{2}^{\circ}$ & $4 d^{8} 5 s^{2}$ & ${ }^{3} \mathrm{P}_{2}$ & K02c & & & & \\
\hline 160 & $3949.4347(5)$ & 25312.915 & $3949.4350(3)$ & -0.0002 & $4 d^{9}{ }_{5 / 2}^{5 p}$ & ${ }^{2}[3 / 2]^{\circ}{ }_{1}$ & $4 d^{8} 5 s^{2}$ & ${ }^{3} \mathrm{P}_{1}$ & K02c & & & & \\
\hline 180 & $3920.1239(5)$ & 25502.176 & $3920.1238(5)$ & 0.0000 & $4 d^{9}{ }_{5 / 2} 5 p$ & ${ }^{2}[3 / 2]^{\circ}{ }_{1}$ & $4 d^{8} 5 s^{2}$ & ${ }^{3} \mathrm{P}_{0}$ & K02c & & & & \\
\hline 140 & 3909.3032(5) & 25572.763 & $3909.30327(20)$ & -0.0001 & $4 d^{9}{ }_{5 / 2} 5 p$ & ${ }^{2}[5 / 2]^{\circ}{ }_{2}$ & $4 d^{8} 5 s^{2}$ & ${ }^{1} \mathrm{D}_{2}$ & K02c & & & & \\
\hline 70 & $3736.516(7)$ & 26755.29 & $3736.5122(20)$ & 0.004 & $4 d^{8} 5 s^{2}$ & ${ }^{3} \mathrm{P}_{2}$ & $4 d^{9}{ }_{5 / 2} 6 p$ & ${ }^{2}[7 / 2]^{\circ}{ }_{3}$ & R40bc & & & & \\
\hline 530 & 3683.3479(4) & 27141.487 & 3683.3481(3) & -0.0002 & $4 d^{9}{ }_{5 / 2}^{5 p}$ & ${ }^{2}[5 / 2]^{\circ}{ }_{3}$ & $4 d^{8} 5 s^{2}$ & ${ }^{1} \mathrm{G}_{4}$ & K02c & $9.9 \mathrm{e}+05$ & $\mathrm{~B}^{+}$ & $\mathrm{C} 05$ & \\
\hline 260 & $3682.4638(4)$ & 27148.003 & $3682.46368(18)$ & 0.0001 & $4 d^{9}{ }_{5 / 2} 5 p$ & ${ }^{2}[3 / 2]^{\circ}{ }_{1}$ & $4 d^{8} 5 s^{2}$ & ${ }^{1} \mathrm{D}_{2}$ & K02c & $9.0 \mathrm{e}+04$ & B & $\mathrm{C} 05$ & \\
\hline 290 & 3614.5495(13) & 27658.077 & $3614.5504(5)$ & -0.0009 & $4 d^{8} 5 s^{2}$ & ${ }^{3} \mathrm{P}_{2}$ & $4 d^{9}{ }_{5 / 2} 6 p$ & ${ }^{2}[5 / 2]^{\circ}{ }_{2}$ & K02c & & & & \\
\hline 290 & 3601.3023(13) & 27759.813 & $3601.3035(4)$ & -0.0012 & $4 d^{8} 5 s^{2}$ & ${ }^{3} \mathrm{P}_{2}$ & $4 d^{9}{ }_{5 / 2} 6 \mathrm{p}$ & ${ }^{2}[5 / 2]^{\circ}{ }_{3}$ & K02c & & & & \\
\hline 82 & $3585.836(8)$ & 27879.54 & $3585.831(4)$ & 0.005 & $4 d^{8} 5 s^{2}$ & ${ }^{3} \mathrm{P}_{2}$ & $4 d^{8}\left({ }^{3} F\right) 5 s 5 p\left({ }^{3} P^{\circ}\right)$ & ${ }^{5} \mathrm{D}_{2}^{\circ}$ & R40bc & & & & \\
\hline 91 & 3583.966(8) & 27894.09 & 3583.9647(18) & 0.001 & $4 d^{8} 5 s^{2}$ & ${ }^{3} \mathrm{P}_{1}$ & $4 d_{3 / 2}^{9} 6 p$ & ${ }^{2}[1 / 2]^{\circ}{ }_{1}$ & R40bc & & & & \\
\hline 150 & $3534.188(7)$ & 28286.96 & 3534.1851(9) & 0.003 & $4 d^{8} 5 s^{2}$ & ${ }^{3} \mathrm{P}_{1}$ & $4 d_{3 / 2}^{9} 6 p$ & ${ }^{2}[3 / 2]^{\circ}{ }_{1}$ & $\mathrm{R} 40 \mathrm{bc}$ & & & & \\
\hline 500 & $3495.2849(4)$ & 28601.787 & $3495.28495(17)$ & 0.0000 & $4 d^{9}{ }_{5 / 2} 5 p$ & ${ }^{2}[7 / 2]^{\circ}{ }_{3}$ & $4 d^{8} 5 s^{2}$ & ${ }^{1} \mathrm{D}_{2}$ & K02c & $7.4 \mathrm{e}+05$ & $\mathrm{C}+$ & C05 & \\
\hline 450 & $3475.8183(4)$ & 28761.969 & $3475.81840(21)$ & -0.0001 & $4 d^{9}{ }_{5 / 2} 5 p$ & ${ }^{2}[3 / 2]_{2}^{\circ}$ & $4 d^{8} 5 s^{2}$ & & K02c & & & & \\
\hline
\end{tabular}


Volume 118 (2013) http://dx.doi.org/10.6028/jres.118.009

Journal of Research of the National Institute of Standards and Technology

\begin{tabular}{|c|c|c|c|c|c|c|c|c|c|c|c|c|c|}
\hline $\begin{array}{l}I_{\text {obs }}{ }^{\mathrm{a}} \\
\text { arb.u. }\end{array}$ & $\begin{array}{c}\lambda_{\text {obs }}{ }^{b} \\
\AA\end{array}$ & $\begin{array}{l}\sigma_{\mathrm{obs}} \\
\mathrm{cm}^{-1}\end{array}$ & $\begin{array}{c}\lambda_{\text {Ritz }} \\
\AA\end{array}$ & $\underset{\AA}{\Delta \lambda_{\text {obs-Ritz }}}$ & Lower leve & & Upper level & & $\begin{array}{l}\text { Line } \\
\text { Ref. }^{c}\end{array}$ & $\begin{array}{l}A \\
\mathrm{~s}^{-1}\end{array}$ & Acc. ${ }^{d}$ & $\begin{array}{l}\text { TP } \\
\text { Ref. }^{\text {e }}\end{array}$ & Note $^{f}$ \\
\hline 150 & $3437.707(7)$ & 29080.82 & 3437.7018(8) & 0.005 & $4 d^{8} 5 s^{2}$ & ${ }^{3} \mathrm{P}_{2}$ & $4 d^{8}\left({ }^{3} F\right) 5 s 5 p\left({ }^{3} P^{\circ}\right)$ & ${ }^{5} \mathrm{G}_{3}^{\circ}$ & R40bc & & & & \multirow{30}{*}{ Las } \\
\hline 2600 & $3384.6244(5)$ & 29536.895 & 3384.6244(5) & 0.0000 & $4 d^{8} 5 s^{2}$ & ${ }^{3} \mathrm{P}_{2}$ & $4 d^{8}\left({ }^{3} F\right) 5 s 5 p\left({ }^{3} P^{\circ}\right)$ & ${ }^{5} \mathrm{D}^{\circ}{ }_{1}$ & K02c & & & & \\
\hline 560 & 3372.1945(5) & 29645.765 & 3372.19464(18) & -0.0002 & $4 d_{3 / 2}^{9} 5 p$ & ${ }^{2}[3 / 2]_{2}^{\circ}$ & $4 d_{5 / 2}^{9} 6 s$ & ${ }^{2}[5 / 2]_{3}$ & K02c & $1.00 \mathrm{e}+06$ & B & $\mathrm{C} 05$ & \\
\hline 88 & 3339.878(7) & 29932.61 & 3339.8913(3) & -0.014 & $4 d^{9}{ }_{5 / 2} 5 p$ & ${ }^{2}[7 / 2]^{\circ}{ }_{4}$ & $4 d^{8} 5 s^{2}$ & ${ }^{1} \mathrm{G}_{4}$ & R40bc & $2.10 \mathrm{e}+05$ & $\mathrm{~B}^{+}$ & $\mathrm{C} 05$ & \\
\hline 90 & $3329.7790(10)$ & 30023.387 & 3329.78173(17) & -0.0027 & $4 d_{3 / 2}^{9} 5 p$ & ${ }^{2}[3 / 2]_{2}^{\circ}$ & $4 d^{9}{ }_{5 / 2} 6 \mathrm{~s}$ & ${ }^{2}[5 / 2]_{2}$ & K02c & & & & \\
\hline 1400 & 3269.55(5) & 30576.4 & $3269.56087(18)$ & -0.01 & $4 d_{3 / 2}^{9} 5 p$ & ${ }^{2}[1 / 2]^{\circ}{ }_{1}$ & $4 d_{5 / 2}^{9} 6 \mathrm{~s}$ & ${ }^{2}[5 / 2]_{2}$ & S28c & $2.0 \mathrm{e}+07$ & $\mathrm{D}^{+}$ & TW & \\
\hline 460 & $3267.3462(3)$ & 30597.058 & $3267.34612(14)$ & 0.0001 & $4 d^{9}{ }_{5 / 2} 5 p$ & ${ }^{2}[3 / 2]^{\circ}{ }_{2}$ & $4 d^{8} 5 s^{2}$ & ${ }^{1} \mathrm{D}_{2}$ & K02c & $8.7 \mathrm{e}^{+05}$ & B & $\mathrm{C} 05$ & \\
\hline 3300 & 3223.2462(3) & 31015.668 & $3223.24625(15)$ & -0.0001 & $4 d_{3 / 2}^{9} 5 p$ & ${ }^{2}[3 / 2]^{\circ}{ }_{1}$ & $4 d^{9}{ }_{5 / 2} 6 s$ & ${ }^{2}[5 / 2]_{2}$ & K02c & $6.9 \mathrm{e}+06$ & $\mathrm{D}^{+}$ & TW & \\
\hline 1300 & 3183.9060(3) & 31398.882 & 3183.90601(15) & 0.0000 & $4 d_{3 / 2}^{9} 5 p$ & ${ }^{2}[5 / 2]^{\circ}{ }_{3}$ & $4 d^{9}{ }_{5 / 2} 6 s$ & ${ }^{2}[5 / 2]_{3}$ & K02c & $4.0 \mathrm{e}+06$ & B & $\mathrm{C} 05$ & \\
\hline 1500 & $3180.7128(3)$ & 31430.403 & $3180.71265(19)$ & 0.0001 & $4 d^{9}{ }_{5 / 2}^{5 p}$ & ${ }^{2}[7 / 2]_{3}^{\circ}$ & $4 d^{8} 5 s^{2}$ & ${ }^{1} \mathrm{G}_{4}$ & K02c & $4.30 \mathrm{e}+06$ & $\mathrm{~B}+$ & C05 & \\
\hline 97 & 3146.0704(8) & 31776.480 & 3146.07035(15) & 0.0001 & $4 d_{3 / 2}^{9} 5 p$ & ${ }^{2}[5 / 2]_{3}^{\circ}$ & $4 d^{9}{ }_{5 / 2} 6 \mathrm{~s}$ & ${ }^{2}[5 / 2]_{2}$ & K02c & & & & \\
\hline 370 & 3127.698(6) & 31963.13 & $3127.6972(7)$ & 0.001 & $4 d^{8} 5 s^{2}$ & ${ }^{3} \mathrm{P}_{2}$ & $4 d_{3 / 2}^{9} 6 p$ & ${ }^{2}[3 / 2]^{\circ}{ }_{1}$ & R40bc & & & & \\
\hline 100 & 3051.3341(19) & 32763.021 & $3051.3340(19)$ & 0.0001 & $4 d^{8} 5 s^{2}$ & ${ }^{3} \mathrm{~F}_{3}$ & $4 d^{8}\left({ }^{3} \mathrm{~F}\right) 5 \mathrm{~s} 5 \mathrm{p}\left({ }^{3} \mathrm{P}^{\circ}\right)$ & ${ }^{5} \mathrm{D}_{3}^{\circ}$ & K02c & & & & \\
\hline 160 & 3007.9395(18) & 33235.664 & 3007.9389(6) & 0.0005 & $4 d^{8} 5 s^{2}$ & ${ }^{3} \mathrm{~F}_{2}$ & $4 d^{9}{ }_{5 / 2} 6 p$ & ${ }^{2}[3 / 2]^{\circ}{ }_{1}$ & K02c & & & & \\
\hline 510 & $3000.8383(6)$ & 33314.309 & $3000.8384(5)$ & -0.0001 & $4 d^{8} 5 s^{2}$ & ${ }^{3} \mathrm{~F}_{2}$ & $4 d^{9}{ }_{5 / 2} 6 p$ & ${ }^{2}[5 / 2]^{\circ}{ }_{2}$ & K02c & & & & \\
\hline 24 & 2981.018(6) & 33535.80 & 2981.016(3) & 0.002 & $4 d^{8} 5 s^{2}$ & ${ }^{3} F_{2}$ & $4 d^{8}\left({ }^{3} F\right) 5 s 5 p\left({ }^{3} P^{\circ}\right)$ & ${ }^{5} \mathrm{D}_{2}^{\circ}$ & R40bc & & & & \\
\hline 8200 & 2938.3197(3) & 34023.106 & 2938.31975(12) & 0.0000 & $4 d_{3 / 2}^{9} 5 p$ & ${ }^{2}[5 / 2]_{2}^{\circ}$ & $4 d^{9}{ }_{5 / 2} 6 \mathrm{~s}$ & ${ }^{2}[5 / 2]_{2}$ & K02c & $3.0 \mathrm{e}+07$ & $\mathrm{D}^{+}$ & TW & \\
\hline 20 & 2935.518(6) & 34055.58 & $2935.5204(12)$ & -0.003 & $4 d^{8} 5 s^{2}$ & ${ }^{3} F_{3}$ & $4 d^{9}{ }_{5 / 2} 6 p$ & ${ }^{2}[7 / 2]_{3}^{\circ}$ & R40bc & & & & \\
\hline 28000 & 2934.0208(3) & 34072.955 & 2934.02078(13) & 0.0000 & $4 d^{9}{ }_{5 / 2} 5 p$ & ${ }^{2}[5 / 2]^{\circ}{ }_{3}$ & $4 d^{9}{ }_{5 / 2} 6 s$ & ${ }^{2}[5 / 2]_{3}$ & K02c & $7.2 \mathrm{e}+07$ & B & $\mathrm{C} 05$ & \\
\hline 1500 & 2933.3102(3) & 34081.208 & 2933.3102(3) & 0.0000 & $4 d^{8} 5 s^{2}$ & ${ }^{3} \mathrm{~F}_{4}$ & $4 d^{8}\left({ }^{3} F\right) 5 s 5 p\left({ }^{3} P^{\circ}\right)$ & ${ }^{5} \mathrm{D}_{4}^{\circ}$ & K02c & & & & \\
\hline 10000 & 2929.3399(3) & 34127.398 & 2929.33993(12) & 0.0000 & $4 d_{3 / 2}^{9} 5 s$ & ${ }^{2}[3 / 2]_{2}$ & $4 d^{9}{ }_{5 / 2} 5 p$ & ${ }^{2}[3 / 2]_{2}^{\circ}$ & K02c & $2.9 \mathrm{e}+06$ & $\mathrm{C}$ & F95c & \\
\hline 8400 & 2919.8283(3) & 34238.567 & 2919.82822(13) & 0.0000 & $4 d_{3 / 2}^{9} 5 p$ & ${ }^{2}[3 / 2]_{2}^{\circ}$ & $4 d^{9}{ }_{3 / 2} 6 s$ & ${ }^{2}[3 / 2]_{1}$ & K02c & $6.0 \mathrm{e}+07$ & $\mathrm{C}^{+}$ & $\mathrm{C} 05$ & \\
\hline 14000 & 2901.8607(3) & 34450.553 & 2901.86068(12) & 0.0001 & $4 d^{9}{ }_{5 / 2} 5 p$ & ${ }^{2}[5 / 2]^{\circ}{ }_{3}$ & $4 d^{9}{ }_{5 / 2} 6 s$ & ${ }^{2}[5 / 2]_{2}$ & K02c & $4.3 \mathrm{e}+07$ & $\mathrm{D}^{+}$ & TW & \\
\hline 12 & $2900.428(6)$ & 34467.57 & $2900.4230(5)$ & 0.005 & $4 d^{8} 5 s^{2}$ & ${ }^{3} \mathrm{~F}_{3}$ & $4 d^{9}{ }_{5 / 2} 6 p$ & ${ }^{2}[7 / 2]^{\circ}{ }_{4}$ & R40bc & & & & \\
\hline 16000 & 2896.2710(3) & 34517.039 & 2896.27093(12) & 0.0000 & $4 d_{3 / 2}^{9} 5 p$ & ${ }^{2}[3 / 2]^{\circ}{ }_{2}$ & $4 d^{9}{ }_{3 / 2} 6 s$ & ${ }^{2}[3 / 2]_{2}$ & K02c & $8.4 \mathrm{e}+07$ & B & $\mathrm{C} 05$ & \\
\hline 760 & $2882.00(5)$ & 34688.0 & 2882.0780(3) & -0.08 & $4 d_{3 / 2}^{9} 5 p$ & ${ }^{2}[3 / 2]^{\circ}{ }_{2}$ & $4 d^{9}{ }_{5 / 2} 5 d$ & ${ }^{2}[1 / 2]_{1}$ & S28c & & & & \\
\hline 9800 & $2878.78902(25)$ & 34726.640 & $2878.78902(25)$ & 0.00000 & $4 d^{8} 5 s^{2}$ & ${ }^{3} \mathrm{~F}_{3}$ & $4 d^{8}\left({ }^{3} \mathrm{~F}\right) 5 \mathrm{~s} 5 \mathrm{p}\left({ }^{3} \mathrm{P}^{\circ}\right)$ & ${ }^{5} \mathrm{G}_{4}^{\circ}$ & K02c & & & & \\
\hline 3800 & 2877.92171(25) & 34737.105 & 2877.9218(3) & -0.00008 & $4 d^{8} 5 s^{2}$ & ${ }^{3} \mathrm{~F}_{2}$ & $4 d^{8}\left({ }^{3} \mathrm{~F}\right) 5 \mathrm{~s} 5 \mathrm{p}\left({ }^{3} \mathrm{P}^{\circ}\right)$ & ${ }^{5} \mathrm{G}^{\circ}{ }_{3}$ & K02c & & & & \\
\hline 11400 & $2873.41877(25)$ & 34791.539 & 2873.41884(13) & -0.00007 & $4 d_{3 / 2}^{9} 5 p$ & ${ }^{2}[1 / 2]^{\circ}{ }_{1}$ & $4 d^{9}{ }_{3 / 2} 6 \mathrm{~s}$ & ${ }^{2}[3 / 2]_{1}$ & K02c & $1.06 \mathrm{e}+08$ & $\mathrm{C}+$ & $\mathrm{C} 05$ & \\
\hline 550 & 2859.7113(7) & 34958.297 & 2859.7111(5) & 0.0002 & $4 d^{8} 5 s^{2}$ & & $4 d^{9}{ }_{5 / 2}^{6} 6 p$ & ${ }^{2}[5 / 2]_{2}^{\circ}$ & K02c & & & & \\
\hline
\end{tabular}


Volume 118 (2013) http://dx.doi.org/10.6028/jres.118.009

Journal of Research of the National Institute of Standards and Technology

\begin{tabular}{|c|c|c|c|c|c|c|c|c|c|c|c|c|c|}
\hline $\begin{array}{c}I_{\mathrm{obs}}^{\mathrm{a}} \\
\text { arb.u. }\end{array}$ & $\begin{array}{c}\lambda_{\text {obs }}^{\mathrm{b}} \\
\AA \\
\end{array}$ & $\begin{array}{c}\begin{array}{c}\sigma_{\mathrm{obs}} \\
\mathrm{cm}^{-1}\end{array} \\
\end{array}$ & $\begin{array}{c}\lambda_{\text {Ritz }} \\
\AA\end{array}$ & $\begin{array}{c}\Delta \lambda_{\text {obs-Ritz }} \\
\AA\end{array}$ & Lower lev & & Upper level & & $\begin{array}{l}\text { Line } \\
\text { Ref. }^{c}\end{array}$ & $\begin{array}{l}A \\
\mathrm{~s}^{-1}\end{array}$ & Acc. $^{\mathrm{d}}$ & $\begin{array}{l}\text { TP } \\
\text { Ref. }^{\text {e }}\end{array}$ & Note $^{f}$ \\
\hline \multirow[t]{2}{*}{410} & 2851.4131(10) & 35060.029 & 2851.4127(5) & 0.0004 & $4 d^{8} 5 s^{2}$ & ${ }^{3} \mathrm{~F}_{3}$ & $4 d^{9}{ }_{5 / 2} 6 p$ & ${ }^{2}[5 / 2]^{\circ}{ }_{3}$ & K02c & & & & \\
\hline & & & $2850.60148(13)$ & & $4 d^{9}{ }_{3 / 2} 5 p$ & ${ }^{2}[1 / 2]^{\circ}{ }_{1}$ & $4 d^{9}{ }_{3 / 2} 6 s$ & ${ }^{2}[3 / 2]_{2}$ & & $1.6 \mathrm{e}+07$ & $\mathrm{D}^{+}$ & TW & $\mathrm{P}$ \\
\hline 180 & $2841.7040(24)$ & 35179.81 & $2841.7040(20)$ & 0.0001 & $4 d^{8} 5 s^{2}$ & ${ }^{3} \mathrm{~F}_{3}$ & $4 d^{8}\left({ }^{3} F\right) 5 s 5 p\left({ }^{3} P^{\circ}\right)$ & ${ }^{5} \mathrm{D}_{2}^{\circ}$ & K02c & & & & \\
\hline 25 & $2840.638(6)$ & 35193.01 & $2840.6282(6)$ & 0.010 & $4 d^{8} 5 s^{2}$ & ${ }^{3} F_{2}$ & $4 d^{8}\left({ }^{3} F\right) 5 s 5 p\left({ }^{3} P^{\circ}\right)$ & ${ }^{5} \mathrm{D}^{\circ}{ }_{1}$ & R40bc & & & & \\
\hline 670 & 2837.57(5) & 35231.1 & 2837.58520(13) & -0.02 & $4 d_{3 / 2}^{9} 5 p$ & ${ }^{2}[3 / 2]^{\circ}{ }_{1}$ & $4 d^{9}{ }_{3 / 2}^{6} 6 s$ & ${ }^{2}[3 / 2]_{1}$ & S28c & $4.0 \mathrm{e}+06$ & B & $\mathrm{C} 05$ & \\
\hline 740 & 2829.0(4) & 35338 & 2829.39497(13) & -0.4 & $4 d^{9} 5 / 2 p$ & ${ }^{2}[5 / 2]_{2}^{\circ}$ & $4 d^{9}{ }_{5 / 2} 6 \mathrm{~s}$ & ${ }^{2}[5 / 2]_{3}$ & S28c & & & & \\
\hline 13000 & 2815.33114(24) & 35509.344 & 2815.33114(11) & 0.00001 & $4 d^{9}{ }_{3 / 2} 5 p$ & ${ }^{2}[3 / 2]^{\circ}{ }_{1}$ & $4 d^{9}{ }_{3 / 2}^{6} 6 s$ & ${ }^{2}[3 / 2]_{2}$ & K02c & $7.1 \mathrm{e}+07$ & $\mathrm{~B}+$ & $\mathrm{C} 05$ & \\
\hline 400 & 2801.93(5) & 35679.2 & $2801.91850(24)$ & 0.01 & $4 d_{3 / 2}^{9} 5 p$ & ${ }^{2}[3 / 2]^{\circ}{ }_{1}$ & $4 d^{9}{ }_{5 / 2} 5 d$ & ${ }^{2}[1 / 2]_{1}$ & B30 & & & & \\
\hline 20000 & 2799.47584(24) & 35710.447 & $2799.47573(11)$ & 0.00012 & $4 d^{9} 5 / 2 p$ & ${ }^{2}[5 / 2]^{\circ}{ }_{2}$ & $4 d^{9}{ }_{5 / 2} 6 s$ & ${ }^{2}[5 / 2]_{2}$ & K02c & $1.0 \mathrm{e}+08$ & $\mathrm{D}^{+}$ & TW & \\
\hline 900 & 2791.7477(8) & 35809.296 & $2791.7480(6)$ & -0.0003 & $4 d^{8} 5 s^{2}$ & ${ }^{3} \mathrm{~F}_{2}$ & $4 d^{8}\left({ }^{3} F\right) 5 s 5 p\left({ }^{3} P^{\circ}\right)$ & ${ }^{5} \mathrm{G}_{2}^{\circ}$ & K02c & & & & \\
\hline 1500 & 2786.31(5) & 35879.2 & 2786.35281(15) & -0.04 & $4 d_{3 / 2}^{9} 5 p$ & ${ }^{2}[3 / 2]_{2}^{\circ}$ & $4 d^{9} 5$ d & ${ }^{2}[3 / 2]_{2}$ & S28c & & & & \\
\hline 30000 & $2767.52713(23)$ & 36122.672 & $2767.52728(11)$ & -0.00015 & $4 d^{9}{ }_{3 / 2} 5 s$ & ${ }^{2}[3 / 2]_{2}$ & $4 d^{9}{ }_{5 / 2} 5 p$ & ${ }^{2}[7 / 2]^{\circ}{ }_{3}$ & K02c & $1.01 \mathrm{e}^{\mathrm{e}+07}$ & B & F95c & \\
\hline 35000 & $2756.27305(23)$ & 36270.156 & 2756.27291(11) & 0.00014 & $4 d^{9}{ }_{3 / 2} 5 p$ & ${ }^{2}[5 / 2]^{\circ}{ }_{3}$ & $4 d_{3 / 2}^{9} 6 s$ & ${ }^{2}[3 / 2]_{2}$ & K02c & $2.0 \mathrm{e}+08$ & $\mathrm{C}+$ & C05 & \\
\hline 690 & 2752.19(5) & 36324.0 & $2752.24198(12)$ & -0.05 & $4 d^{9}{ }_{3 / 2} 5 p$ & ${ }^{2}[3 / 2]^{\circ}{ }_{2}$ & $4 d^{9}{ }_{5 / 2} 5 d$ & ${ }^{2}[5 / 2]_{3}$ & В30 & $1.0 \mathrm{e}+06$ & E & $\mathrm{C} 05$ & \\
\hline 1290 & 2743.8981(3) & 36433.725 & $2743.89810(11)$ & 0.0000 & $4 d^{9}{ }_{3 / 2} 5 \mathrm{~s}$ & ${ }^{2}[3 / 2]_{1}$ & $4 d^{9}{ }_{5 / 2} 5 p$ & ${ }^{2}[3 / 2]^{\circ}{ }_{2}$ & K02c & $1.1 \mathrm{e}^{+}+06$ & $\mathrm{D}^{+}$ & F95c & \\
\hline 5000 & 2743.7697(5) & 36435.430 & $2743.76993(14)$ & -0.0002 & $4 d^{9}{ }_{3 / 2} 5 p$ & ${ }^{2}[1 / 2]^{\circ}{ }_{1}$ & $4 d^{9}{ }_{5 / 2}^{5 d}$ & ${ }^{2}[3 / 2]_{1}$ & K02c & $1.0 \mathrm{e}+08$ & $\mathrm{D}^{+}$ & TW & \\
\hline 590 & 2728.73(4) & 36636.3 & 2728.77443(13) & -0.05 & $4 d^{9}{ }_{3 / 2} 5 p$ & ${ }^{2}[3 / 2]^{\circ}{ }_{2}$ & $4 d^{9}{ }_{5 / 2} 5 d$ & ${ }^{2}[5 / 2]_{2}$ & B30 & $1.0 \mathrm{e}+06$ & $\mathrm{E}$ & $\mathrm{C} 05$ & \\
\hline 91000 & $2711.87305(22)$ & 36863.955 & 2711.87292(13) & 0.00013 & $4 d^{9} 5 / 2 p$ & ${ }^{2}[7 / 2]^{\circ}{ }_{4}$ & $4 d^{9}{ }_{5 / 2}^{6} 6 s$ & ${ }^{2}[5 / 2]_{3}$ & K02c & $2.0 \mathrm{e}+08$ & $\mathrm{C}+$ & $\mathrm{C} 05$ & \\
\hline 8900 & $2711.07823(22)$ & 36874.762 & $2711.07830(12)$ & -0.00007 & $4 d^{9} 3 / 25 p$ & ${ }^{2}[3 / 2]^{\circ}{ }_{1}$ & $4 d^{9}{ }_{5 / 2}^{5} \mathrm{~d}$ & ${ }^{2}[3 / 2]_{1}$ & K02c & $4.8 \mathrm{e}+07$ & $\mathrm{D}^{+}$ & TW & \\
\hline 2300 & 2688.1976(6) & 37188.605 & $2688.19709(13)$ & 0.0005 & $4 d^{9}{ }_{3 / 2} 5 p$ & ${ }^{2}[1 / 2]^{\circ}{ }_{1}$ & $4 d^{9}{ }_{5 / 2}^{5 d}$ & ${ }^{2}[5 / 2]_{2}$ & K02c & $1.40 \mathrm{e}+07$ & $\mathrm{~B}+$ & $\mathrm{C} 05$ & \\
\hline 24000 & $2681.19774(22)$ & 37285.688 & $2681.19759(10)$ & 0.00015 & $4 d^{9}{ }_{5 / 2} 5 p$ & ${ }^{2}[3 / 2]^{\circ}{ }_{1}$ & $4 d^{9}{ }_{5 / 2} 6 s$ & ${ }^{2}[5 / 2]_{2}$ & K02c & $6.0 \mathrm{e}+07$ & $\mathrm{D}^{+}$ & TW & \\
\hline 2100 & 2669.1994(5) & 37453.282 & 2669.1992(4) & 0.0001 & $4 d^{8} 5 s^{2}$ & ${ }^{3} \mathrm{~F}_{3}$ & $4 d^{8}\left({ }^{3} \mathrm{~F}\right) 5 \mathrm{~s} 5 \mathrm{p}\left({ }^{3} \mathrm{P}^{\circ}\right)$ & ${ }^{5} \mathrm{G}_{2}^{\circ}$ & К02с & & & & \\
\hline 39000 & $2663.17552(21)$ & 37537.993 & & & $4 d^{8} 5 s^{2}$ & ${ }^{3} \mathrm{~F}_{4}$ & $4 d^{8}\left({ }^{3} \mathrm{~F}\right) 5 \mathrm{~s} 5 \mathrm{p}\left({ }^{3} \mathrm{P}^{\circ}\right)$ & ${ }^{5} \mathrm{G}_{5}^{\circ}$ & K02c & & & & S \\
\hline 26000 & $2660.44956(21)$ & 37576.453 & $2660.44959(9)$ & -0.00002 & $4 d_{3 / 2}^{9} 5 s$ & ${ }^{2}[3 / 2]_{2}$ & $4 d^{9} 5 p$ & ${ }^{2}[3 / 2]^{\circ}{ }_{1}$ & K02c & $1.51 \mathrm{e}+07$ & $\mathrm{C}^{+}$ & F95c & \\
\hline 7000 & 2657.4137(3) & 37619.379 & $2657.4137(3)$ & -0.0001 & $4 d^{8} 5 s^{2}$ & ${ }^{3} \mathrm{~F}_{2}$ & $4 d^{9}{ }_{3 / 2} 6 p$ & ${ }^{2}[3 / 2]^{\circ}{ }_{1}$ & K02c & & & & \\
\hline 6200 & $2656.80859(21)$ & 37627.946 & $2656.80863(11)$ & -0.00004 & $4 d_{3 / 2}^{9} 5 p$ & ${ }^{2}[3 / 2]^{\circ}{ }_{1}$ & $4 d^{9}{ }_{5 / 2} 5 d$ & ${ }^{2}[5 / 2]_{2}$ & K02c & $2.90 \mathrm{e}+07$ & $\mathrm{~B}^{+}$ & $\mathrm{C} 05$ & \\
\hline 2500 & $2656.5404(4)$ & 37631.745 & $2656.54043(13)$ & -0.0001 & $4 d^{9} 3 / 25 p$ & ${ }^{2}[5 / 2]^{\circ}{ }_{3}$ & $4 d^{9}{ }_{5 / 2} 5 d$ & ${ }^{2}[3 / 2]_{2}$ & К02c & $1.3 \mathrm{e}+07$ & $\mathrm{D}+$ & TW & \\
\hline 8200 & $2625.51589(21)$ & 38076.395 & $2625.51592(10)$ & -0.00003 & $4 d^{9} 3 / 25$ & ${ }^{2}[5 / 2]_{3}^{\circ}$ & $4 d^{9}{ }_{5 / 2}^{5} 5 d$ & ${ }^{2}[5 / 2]_{3}$ & K02c & $3.00 \mathrm{e}+07$ & $\mathrm{~B}^{+}$ & $\mathrm{C} 05$ & \\
\hline 20 & $2624.947(6)$ & 38084.64 & $2624.9459(10)$ & 0.002 & $4 d^{8} 5 s^{2}$ & & $4 d^{9}{ }_{5 / 2}^{6} 6 p$ & ${ }^{2}[7 / 2]^{\circ}{ }_{3}$ & R40bc & & & & \\
\hline 3600 & $2617.0020(3)$ & 38200.261 & $2617.00202(21)$ & 0.0000 & $4 d_{3 / 2}^{9} 5 p$ & ${ }^{2}[1 / 2]^{\circ}{ }_{1}$ & $4 d^{9}{ }_{5 / 2}^{5 d}$ & ${ }^{2}[1 / 2]_{0}$ & K02c & $6.9 \mathrm{e}+07$ & $\mathrm{~B}^{+}$ & $\mathrm{C} 05$ & \\
\hline
\end{tabular}


Volume 118 (2013) http://dx.doi.org/10.6028/jres.118.009

Journal of Research of the National Institute of Standards and Technology

\begin{tabular}{|c|c|c|c|c|c|c|c|c|c|c|c|c|c|}
\hline $\begin{array}{c}I_{\mathrm{obs}}^{\mathrm{a}} \\
\text { arb.u. }\end{array}$ & $\begin{array}{c}\lambda_{\text {obs }}{ }^{2} \\
\AA \\
\end{array}$ & $\begin{array}{c}\begin{array}{c}\sigma_{\mathrm{obs}} \\
\mathrm{cm}^{-1}\end{array} \\
\end{array}$ & $\begin{array}{c}\lambda_{\text {Ritz }} \\
\AA\end{array}$ & $\begin{array}{c}\Delta \lambda_{\text {obs-Ritz }} \\
\AA\end{array}$ & Lower level & & Upper level & & $\begin{array}{l}\text { Line } \\
\text { Ref. }^{c}\end{array}$ & $\begin{array}{l}A \\
\mathrm{~s}^{-1}\end{array}$ & Acc. ${ }^{\mathrm{d}}$ & $\begin{array}{l}\text { TP } \\
\text { Ref. }\end{array}$ & Note $^{f}$ \\
\hline 21000 & 2614.39776(21) & 38238.311 & 2614.39778(10) & -0.00002 & $4 d_{3 / 2}^{9} 5 p$ & ${ }^{2}[5 / 2]_{2}^{\circ}$ & $4 d^{9}{ }_{3 / 2} 6 \mathrm{~s}$ & ${ }^{2}[3 / 2]_{1}$ & K02c & $1.36 \mathrm{e}+08$ & $\mathrm{C}+$ & $\mathrm{C} 05$ & \\
\hline 24000 & 2605.97631(20) & 38361.874 & 2605.97632(10) & -0.00001 & $4 d^{9}{ }_{5 / 2} 5 p$ & ${ }^{2}[7 / 2]^{\circ}{ }_{3}$ & $4 d^{9}{ }_{5 / 2} 6 s$ & ${ }^{2}[5 / 2]_{3}$ & K02c & $3.0 \mathrm{e}+07$ & $\mathrm{C}+$ & $\mathrm{C} 05$ & \\
\hline 3400 & 2598.36(5) & 38474.3 & 2598.38439(15) & -0.02 & $4 d_{3 / 2}^{9} 5 p$ & ${ }^{2}[5 / 2]_{3}^{\circ}$ & $4 d^{9}{ }_{5 / 2} 5 d$ & ${ }^{2}[7 / 2]_{4}$ & S28c & $8.0 \mathrm{e}+06$ & $\mathrm{C}+$ & $\mathrm{C} 05$ & \\
\hline 24000 & $2596.84600(20)$ & 38496.743 & $2596.84607(20)$ & -0.00007 & $4 d^{8} 5 s^{2}$ & ${ }^{3} \mathrm{~F}_{4}$ & $4 d^{9}{ }_{5 / 2}^{6} 6 p$ & ${ }^{2}[7 / 2]^{\circ}{ }_{4}$ & K02c & & & & \\
\hline 8100 & 2595.49487(20) & 38516.782 & 2595.49481(9) & 0.00005 & $4 d^{9}{ }_{3 / 2}^{5 p}$ & ${ }^{2}[5 / 2]_{2}^{\circ}$ & $4 d^{9}{ }_{3 / 2}^{6} 6 s$ & ${ }^{2}[3 / 2]_{2}$ & K02c & $2.8 \mathrm{e}+07$ & $\mathrm{C}+$ & $\mathrm{C} 05$ & \\
\hline 1100 & 2587.24(4) & 38639.7 & $2587.24477(20)$ & -0.01 & $4 d^{9}{ }_{3 / 2}^{5} p$ & ${ }^{2}[3 / 2]^{\circ}{ }_{1}$ & $4 d^{9}{ }_{5 / 2} 5 d$ & ${ }^{2}[1 / 2]_{0}$ & B30 & $2.0 \mathrm{e}+06$ & E & $\mathrm{C} 05$ & \\
\hline 1400 & 2584.0917(10) & 38686.739 & $2584.09063(20)$ & 0.0011 & $4 d_{3 / 2}^{9} 5 p$ & ${ }^{2}[5 / 2]^{\circ}{ }_{2}$ & $4 d^{9}{ }_{5 / 2}^{5}$ d & ${ }^{2}[1 / 2]_{1}$ & K02c & & & & \\
\hline 80000 & $2580.57399(20)$ & 38739.472 & 2580.57394(10) & 0.00005 & $4 d^{9}{ }_{5 / 2} 5 p$ & ${ }^{2}[7 / 2]^{\circ}{ }_{3}$ & $4 d^{9}{ }_{5 / 2} 6 s$ & ${ }^{2}[5 / 2]_{2}$ & K02c & $1.8 \mathrm{e}+08$ & $\mathrm{C}$ & TW & \\
\hline 6400 & $2567.00519(20)$ & 38944.230 & 2567.00514(9) & 0.00005 & $4 d^{9}{ }_{5 / 2} 5 p$ & ${ }^{2}[5 / 2]_{3}^{\circ}$ & $4 d_{3 / 2}^{9} 6 s$ & ${ }^{2}[3 / 2]_{2}$ & K02c & $2.5 \mathrm{e}+07$ & $\mathrm{C}^{+}$ & $\mathrm{C} 05$ & \\
\hline 12000 & 2564.23332(20) & 38986.325 & $2564.23336(12)$ & -0.00004 & $4 d_{3 / 2}^{9} 5 p$ & ${ }^{2}[1 / 2]_{0}^{\circ}$ & $4 d_{3 / 2}^{9} 6 s$ & ${ }^{2}[3 / 2]_{1}$ & K02c & $6.2 \mathrm{e}+07$ & $\mathrm{C}^{+}$ & $\mathrm{C} 05$ & \\
\hline 66000 & $2557.48774(20)$ & 39089.148 & $2557.48780(21)$ & -0.00007 & $4 d^{8} 5 s^{2}$ & ${ }^{3} \mathrm{~F}_{4}$ & $4 d^{9}{ }_{5 / 2} 6 p$ & ${ }^{2}[5 / 2]_{3}^{\circ}$ & K02c & & & & \\
\hline 2800 & 2553.4021(7) & 39151.689 & 2553.40179(9) & 0.0003 & $4 d_{3 / 2}^{9} 5 s$ & ${ }^{2}[3 / 2]_{2}$ & $4 d^{9}{ }_{5 / 2} 5 p$ & ${ }^{2}[5 / 2]^{\circ}{ }_{2}$ & K02c & 7.e+05 & E & F95c & \\
\hline 19000 & 2535.30785(19) & 39431.093 & 2535.30785(8) & 0.00000 & $4 d^{9}{ }_{5 / 2} 5 s$ & ${ }^{2}[5 / 2]_{2}$ & $4 d^{9}{ }_{5 / 2} 5 p$ & ${ }^{2}[3 / 2]_{2}^{\circ}$ & K02c & $3.4 \mathrm{e}+06$ & E & F95c & \\
\hline 390 & $2535.07(4)$ & 39434.8 & 2535.07148(19) & 0.00 & $4 d^{9}{ }_{3 / 2} 5 p$ & ${ }^{2}[1 / 2]^{\circ}{ }_{0}$ & $4 d^{9}{ }_{5 / 2} 5 d$ & ${ }^{2}[1 / 2]_{1}$ & В30 & & & & \\
\hline 6300 & $2532.6045(6)$ & 39473.180 & 2532.6044(6) & 0.0001 & $4 d^{8} 5 s^{2}$ & ${ }^{3} \mathrm{~F}_{3}$ & $4 d^{8}\left({ }^{3} F\right) 5 s 5 p\left({ }^{3} P^{\circ}\right)$ & ${ }^{5} \mathrm{~F}_{3}^{\circ}$ & K02c & & & & \\
\hline 4300 & $2530.4869(5)$ & 39506.210 & 2530.4865(4) & 0.0004 & $4 d^{8} 5 s^{2}$ & ${ }^{3} F_{3}$ & $4 d_{3 / 2}^{9} 6 p$ & ${ }^{2}[3 / 2]_{2}^{\circ}$ & K02c & & & & \\
\hline 12000 & $2507.0909(3)$ & 39874.855 & $2507.09088(11)$ & 0.0000 & $4 d_{3 / 2}^{9} 5 p$ & ${ }^{2}[3 / 2]_{2}^{\circ}$ & $4 d_{3 / 2}^{9} 5 d$ & ${ }^{2}[1 / 2]_{1}$ & K02c & $9 . e+07$ & $\mathrm{D}^{+}$ & TW & \\
\hline 290 & 2506.91(4) & 39877.8 & 2506.86992(12) & 0.04 & $4 d^{9}{ }_{3 / 2}^{5} p$ & ${ }^{2}[5 / 2]_{2}^{\circ}$ & $4 d^{9}{ }_{5 / 2} 5 d$ & ${ }^{2}[3 / 2]_{2}$ & B30 & & & & \\
\hline 5300 & 2506.6289(3) & 39882.204 & $2506.62912(10)$ & -0.0003 & $4 d^{9}{ }_{3 / 2}^{5} p$ & ${ }^{2}[5 / 2]^{\circ}{ }_{2}$ & $4 d^{9}{ }_{5 / 2}^{5} \mathrm{~d}$ & ${ }^{2}[3 / 2]_{1}$ & K02c & $4.6 \mathrm{e}+07$ & $\mathrm{D}^{+}$ & TW & \\
\hline 50000 & $2506.59265(19)$ & 39882.780 & 2506.59265(9) & -0.00001 & $4 d^{9}{ }_{3 / 2} 5 s$ & ${ }^{2}[3 / 2]_{1}$ & $4 d^{9}{ }_{5 / 2}^{5 p}$ & ${ }^{2}[3 / 2]^{\circ}{ }_{1}$ & K02c & $2.13 e+07$ & B & F95c & \\
\hline 17000 & 2503.90077(19) & 39925.654 & 2503.90081(9) & -0.00004 & $4 d^{9}{ }_{5 / 2}^{5 p}$ & ${ }^{2}[5 / 2]^{\circ}{ }_{2}$ & $4 d_{3 / 2}^{9} 6 s$ & ${ }^{2}[3 / 2]_{1}$ & K02c & $9.2 \mathrm{e}+07$ & B & $\mathrm{C} 05$ & \\
\hline 5700 & $2486.55629(19)$ & 40204.128 & 2486.55645(9) & -0.00015 & $4 d^{9}{ }_{5 / 2}^{5 p}$ & ${ }^{2}[5 / 2]^{\circ}{ }_{2}$ & $4 d_{3 / 2}^{9} 6 s$ & ${ }^{2}[3 / 2]_{2}$ & K02c & $1.60 \mathrm{e}+07$ & $\mathrm{C}+$ & $\mathrm{C} 05$ & \\
\hline 9300 & $2485.65135(25)$ & 40218.764 & $2485.65135(12)$ & 0.00000 & $4 d^{9}{ }_{5 / 2}^{5 p}$ & ${ }^{2}[5 / 2]_{3}^{\circ}$ & $4 d^{9}{ }_{5 / 2} 5 d$ & ${ }^{2}[9 / 2]_{4}$ & K02c & $2.8 \mathrm{e}+07$ & $\mathrm{D}^{+}$ & TW & \\
\hline 29000 & $2480.28243(18)$ & 40305.817 & $2480.28243(11)$ & 0.00000 & $4 d^{9}{ }_{5 / 2} 5 p$ & ${ }^{2}[5 / 2]^{\circ}{ }_{3}$ & $4 d^{9}{ }_{5 / 2} 5 d$ & ${ }^{2}[3 / 2]_{2}$ & K02c & $1.6 \mathrm{e}+08$ & $\mathrm{C}$ & TW & \\
\hline 1900 & 2479.2251(4) & 40323.005 & $2479.22422(10)$ & 0.0009 & $4 d_{3 / 2}^{9} 5 p$ & ${ }^{2}[5 / 2]_{2}^{\circ}$ & $4 d^{9}{ }_{5 / 2} 5 d$ & ${ }^{2}[5 / 2]_{3}$ & K02c & $3.0 \mathrm{e}+06$ & E & $\mathrm{C} 05$ & \\
\hline 100000 & 2477.12768(18) & 40357.145 & 2477.12763(9) & 0.00004 & $4 d^{9}{ }_{5 / 2} 5 p$ & ${ }^{2}[3 / 2]^{\circ}{ }_{2}$ & $4 d^{9}{ }_{5 / 2} 6 s$ & ${ }^{2}[5 / 2]_{3}$ & K02c & $1.4 \mathrm{e}+08$ & $\mathrm{C}$ & $\mathrm{C} 05$ & \\
\hline 7200 & 2476.61(5) & 40365.5 & $2476.66649(13)$ & -0.05 & $4 d_{3 / 2}^{9} 5 p$ & ${ }^{2}[3 / 2]_{2}^{\circ}$ & $4 d_{3 / 2}^{9} 5 d$ & ${ }^{2}[7 / 2]_{3}$ & $\mathrm{~S} 28 \mathrm{c}$ & $2.2 \mathrm{e}+07$ & $\mathrm{D}^{+}$ & TW & \\
\hline 3600 & $2476.09(5)$ & 40374.1 & $2476.08744(18)$ & 0.00 & $4 d^{9} 5 / 2 p$ & ${ }^{2}[5 / 2]_{2}^{\circ}$ & $4 d^{9}{ }_{5 / 2}^{5} 5 d$ & ${ }^{2}[1 / 2]_{1}$ & $\mathrm{~S} 28 \mathrm{c}$ & & & & \\
\hline 140000 & $2473.79016(18)$ & 40411.589 & $2473.79016(9)$ & 0.00001 & $4 d_{3 / 2}^{9} 5 \mathrm{~s}$ & ${ }^{2}[3 / 2]_{2}$ & $4 d^{9}{ }_{5 / 2}^{5 p}$ & ${ }^{2}[5 / 2]_{3}^{\circ}$ & K02c & $4.98 \mathrm{e}+07$ & A & F95c & \\
\hline 9700 & $2472.79642(24)$ & 40427.828 & $2472.79659(12)$ & -0.00017 & $4 d_{3 / 2}^{9} 5 p$ & ${ }^{2}[1 / 2]^{\circ}{ }_{1}$ & $4 d_{3 / 2}^{9} 5 d$ & ${ }^{2}[1 / 2]_{1}$ & K02c & $2.3 \mathrm{e}+08$ & $\mathrm{D}^{+}$ & TW & \\
\hline
\end{tabular}


Volume 118 (2013) http://dx.doi.org/10.6028/jres.118.009

Journal of Research of the National Institute of Standards and Technology

\begin{tabular}{|c|c|c|c|c|c|c|c|c|c|c|c|c|c|}
\hline $\begin{array}{c}I_{\text {obs }}^{\mathrm{a}} \\
\text { arb.u. }\end{array}$ & $\begin{array}{c}\lambda_{\text {obs }}{ }^{b} \\
\AA\end{array}$ & $\begin{array}{c}\begin{array}{c}\sigma_{\mathrm{obs}} \\
\mathrm{cm}^{-1}\end{array} \\
\mathrm{c}\end{array}$ & $\begin{array}{c}\lambda_{\text {Ritz }} \\
\AA\end{array}$ & $\begin{array}{c}\Delta \lambda_{\text {obs-Ritz }} \\
\AA\end{array}$ & Lower level & & Upper level & & $\begin{array}{l}\text { Line } \\
\text { Ref. }^{c}\end{array}$ & $\begin{array}{l}A \\
\mathrm{~s}^{-1}\end{array}$ & Acc. ${ }^{\mathrm{d}}$ & $\begin{array}{l}\text { TP } \\
\text { Ref. }^{\text {e }}\end{array}$ & Note $^{f}$ \\
\hline 35000 & 2462.12773(18) & 40602.994 & $2462.12763(10)$ & 0.00010 & $4 d_{3 / 2}^{9} 5 p$ & ${ }^{2}[5 / 2]_{2}^{\circ}$ & $4 d^{9}{ }_{5 / 2} 5 d$ & ${ }^{2}[7 / 2]_{3}$ & K02c & $8 . e+07$ & $\mathrm{D}^{+}$ & TW & \\
\hline 5500 & 2461.1412(3) & 40619.269 & $2461.14111(11)$ & 0.0000 & $4 d^{9}{ }_{3 / 2} 5 p$ & ${ }^{2}[3 / 2]^{\circ}{ }_{2}$ & $4 d^{9}{ }_{3 / 2} 5 d$ & ${ }^{2}[3 / 2]_{1}$ & K02c & $3.4 \mathrm{e}+07$ & $\mathrm{D}^{+}$ & TW & \\
\hline 34000 & 2460.16512(18) & 40635.383 & $2460.16505(9)$ & 0.00007 & $4 d_{3 / 2}^{9} 5 p$ & ${ }^{2}[5 / 2]^{\circ}{ }_{2}$ & $4 d^{9}{ }_{5 / 2}^{5} \mathrm{~d}$ & ${ }^{2}[5 / 2]_{2}$ & K02c & $1.18 \mathrm{e}+08$ & $\mathrm{~B}+$ & $\mathrm{C} 05$ & \\
\hline 680 & 2454.1636(4) & 40734.747 & 2454.16376(9) & -0.0001 & $4 d^{9}{ }_{5 / 2}^{5 p}$ & ${ }^{2}[3 / 2]_{2}^{\circ}$ & $4 d^{9}{ }_{5 / 2}^{6} 6 s$ & ${ }^{2}[5 / 2]_{2}$ & K02c & & & & \\
\hline 110000 & 2453.21676(18) & 40750.468 & 2453.21679(8) & -0.00003 & $4 d^{9}{ }_{5 / 2} 5 p$ & ${ }^{2}[5 / 2]_{3}^{\circ}$ & $4 d^{9}{ }_{5 / 2} 5 d$ & ${ }^{2}[5 / 2]_{3}$ & K02c & $3.7 \mathrm{e}+08$ & $\mathrm{~B}^{+}$ & $\mathrm{C} 05$ & \\
\hline 210000 & 2447.89606(18) & 40839.036 & $2447.89600(8)$ & 0.00006 & $4 d^{9}{ }_{3 / 2} 5 \mathrm{~s}$ & ${ }^{2}[3 / 2]_{2}$ & $4 d^{9}{ }_{3 / 2}^{5} p$ & ${ }^{2}[5 / 2]_{2}^{\circ}$ & K02c & $9.2 \mathrm{e}+07$ & $\mathrm{~B}^{+}$ & F95c & \\
\hline 22000 & 2446.21146(18) & 40867.158 & 2446.21139(11) & 0.00007 & $4 d_{3 / 2}^{9} 5 p$ & ${ }^{2}[3 / 2]^{\circ}{ }_{1}$ & $4 d_{3 / 2}^{9} 5 d$ & ${ }^{2}[1 / 2]_{1}$ & K02c & $3.5 \mathrm{e}+07$ & $\mathrm{D}^{+}$ & TW & \\
\hline 140000 & 2444.09896(18) & 40902.478 & 2444.09897(10) & -0.00001 & $4 d_{3 / 2}^{9} 5 p$ & ${ }^{2}[3 / 2]_{2}^{\circ}$ & $4 d_{3 / 2}^{9} 5 d$ & ${ }^{2}[3 / 2]_{2}$ & K02c & $2.7 \mathrm{e}+08$ & C & TW & \\
\hline 450000 & 2437.78299(18) & 41008.443 & 2437.78303(9) & -0.00004 & $4 d^{9}{ }_{5 / 2} 5 s$ & ${ }^{2}[5 / 2]_{3}$ & $4 d^{9}{ }_{5 / 2} 5 p$ & ${ }^{2}[3 / 2]^{\circ}{ }_{2}$ & K02c & $2.90 \mathrm{e}+08$ & B & F95c & \\
\hline 12000 & $2436.47567(24)$ & 41030.445 & $2436.47576(10)$ & -0.00009 & $4 d^{9}{ }_{5 / 2}^{5 p}$ & ${ }^{2}[5 / 2]_{3}^{\circ}$ & $4 d^{9}{ }_{5 / 2}^{5 d}$ & ${ }^{2}[7 / 2]_{3}$ & K02c & & & & \\
\hline 190000 & 2429.51289(18) & 41148.026 & $2429.51294(12)$ & -0.00005 & $4 d^{9}{ }_{5 / 2} 5 p$ & ${ }^{2}[5 / 2]^{\circ}{ }_{3}$ & $4 d^{9}{ }_{5 / 2} 5 d$ & ${ }^{2}[7 / 2]_{4}$ & K02c & $4.0 \mathrm{e}+08$ & $\mathrm{~B}^{+}$ & $\mathrm{C} 05$ & \\
\hline 30000 & 2428.08389(18) & 41172.241 & 2428.08393(11) & -0.00004 & $4 d_{3 / 2}^{9} 5 p$ & ${ }^{2}[1 / 2]^{\circ}{ }_{1}$ & $4 d^{9}{ }_{3 / 2} 5 d$ & ${ }^{2}[3 / 2]_{1}$ & K02c & & & & \\
\hline 41000 & $2422.46274(18)$ & 41267.771 & $2422.46271(9)$ & 0.00003 & $4 d_{3 / 2}^{9} 5 p$ & ${ }^{2}[3 / 2]_{2}^{\circ}$ & $4 d_{3 / 2}^{9} 5 d$ & ${ }^{2}[5 / 2]_{2}$ & $\mathrm{~K} 02 \mathrm{c}$ & $5.2 \mathrm{e}+07$ & $\mathrm{D}^{+}$ & TW & \\
\hline 540000 & 2419.95667(18) & 41310.504 & 2419.95662(11) & 0.00005 & $4 d^{9}{ }_{3 / 2} 5 p$ & ${ }^{2}[3 / 2]_{2}^{\circ}$ & $4 d^{9}{ }_{3 / 2} 5 d$ & ${ }^{2}[5 / 2]_{3}$ & K02c & $4.6 \mathrm{e}+08$ & $\mathrm{C}$ & TW & \\
\hline 470000 & $2413.18810(17)$ & 41426.364 & 2413.18805(8) & 0.00005 & $4 d^{9} 5 / 25 s$ & ${ }^{2}[5 / 2]_{2}$ & $4 d^{9}{ }_{5 / 2} 5 p$ & ${ }^{2}[7 / 2]^{\circ}{ }_{3}$ & K02c & $2.10 \mathrm{e}+08$ & $\mathrm{~B}^{+}$ & F95c & \\
\hline 91000 & 2411.49494(17) & 41455.448 & 2411.49491(10) & 0.00003 & $4 d_{3 / 2}^{9} 5 p$ & ${ }^{2}[1 / 2]^{\circ}{ }_{1}$ & $4 d_{3 / 2}^{9} 5 d$ & ${ }^{2}[3 / 2]_{2}$ & K02c & $2.5 \mathrm{e}+08$ & C & TW & \\
\hline 180000 & 2411.34527(17) & 41458.021 & $2411.34524(8)$ & 0.00003 & $4 d_{3 / 2}^{9} 5 s$ & ${ }^{2}[3 / 2]_{1}$ & $4 d^{9}{ }_{5 / 2}^{5 p}$ & ${ }^{2}[5 / 2]_{2}^{\circ}$ & K02c & $9.6 \mathrm{e}+07$ & $\mathrm{~B}^{+}$ & F95c & \\
\hline 5900 & 2408.85401(17) & 41500.894 & 2408.85395(8) & 0.00006 & $4 d^{9}{ }_{5 / 2}^{5} p$ & ${ }^{2}[3 / 2]^{\circ}{ }_{1}$ & $4 d^{9}{ }_{3 / 2}^{6} 6 s$ & ${ }^{2}[3 / 2]_{1}$ & K02c & $1.7 \mathrm{e}+07$ & $\mathrm{C}+$ & $\mathrm{C} 05$ & \\
\hline 15000 & 2404.87583(17) & 41569.540 & 2404.87569(9) & 0.00014 & $4 d^{9}{ }_{5 / 2}^{5} p$ & ${ }^{2}[5 / 2]^{\circ}{ }_{2}$ & $4 d^{9}{ }_{5 / 2}^{5} \mathrm{~d}$ & ${ }^{2}[3 / 2]_{1}$ & K02c & $1.1 \mathrm{e}^{+}+08$ & $\mathrm{D}^{+}$ & TW & \\
\hline 31000 & $2402.44630(17)$ & 41611.575 & $2402.44634(10)$ & -0.00004 & $4 d_{3 / 2}^{9} 5 p$ & ${ }^{2}[3 / 2]^{\circ}{ }_{1}$ & $4 d_{3 / 2}^{9} 5 d$ & ${ }^{2}[3 / 2]_{1}$ & K02c & $3.6 \mathrm{e}+08$ & C & TW & \\
\hline 11000 & 2392.79686(17) & 41779.369 & 2392.79697(8) & -0.00011 & $4 d^{9}{ }_{5 / 2}^{5 p}$ & ${ }^{2}[3 / 2]^{\circ}{ }_{1}$ & $4 d_{3 / 2}^{9} 6 s$ & ${ }^{2}[3 / 2]_{2}$ & K02c & $2.50 \mathrm{e}+07$ & B & $\mathrm{C} 05$ & \\
\hline 310000 & $2390.42949(17)$ & 41820.742 & 2390.42948(9) & 0.00001 & $4 d_{3 / 2}^{9} 5 p$ & ${ }^{2}[1 / 2]^{\circ}{ }_{1}$ & $4 d_{3 / 2}^{9} 5 d$ & ${ }^{2}[5 / 2]_{2}$ & K02c & $6.8 \mathrm{e}+07$ & $\mathrm{D}^{+}$ & TW & \\
\hline 32000 & $2386.20459(17)$ & 41894.782 & $2386.20456(9)$ & 0.00003 & $4 d_{3 / 2}^{9} 5 p$ & ${ }^{2}[3 / 2]^{\circ}{ }_{1}$ & $4 d_{3 / 2}^{9} 5 d$ & ${ }^{2}[3 / 2]_{2}$ & K02c & & & & \\
\hline 14000 & 2383.10087(23) & 41949.341 & 2383.10093(14) & -0.00006 & $4 d^{9}{ }_{5 / 2} 5 p$ & ${ }^{2}[3 / 2]^{\circ}{ }_{1}$ & $4 d^{9}{ }_{5 / 2} 5 d$ & ${ }^{2}[1 / 2]_{1}$ & K02c & $8 . e+07$ & $\mathrm{D}^{+}$ & TW & \\
\hline 2800 & $2379.60(5)$ & 42011.0 & 2379.63907(9) & -0.04 & $4 d^{9}{ }_{5 / 2} 5 p$ & ${ }^{2}[5 / 2]_{2}^{\circ}$ & $4 d^{9}{ }_{5 / 2} 5 d$ & ${ }^{2}[5 / 2]_{3}$ & S28c & $9 . e+05$ & $\mathrm{E}$ & $\mathrm{C} 05$ & \\
\hline 16000 & 2373.5695(3) & 42117.781 & $2373.56965(12)$ & -0.0002 & $4 d_{3 / 2}^{9} 5 p$ & ${ }^{2}[5 / 2]^{\circ}{ }_{3}$ & $4 d^{9}{ }_{3 / 2} 5 d$ & ${ }^{2}[7 / 2]_{3}$ & $\mathrm{~K} 02 \mathrm{c}$ & $3.7 \mathrm{e}+07$ & $\mathrm{D}^{+}$ & TW & \\
\hline 9800 & $2370.3984(4)$ & 42174.121 & $2370.3981(4)$ & 0.0003 & $4 d^{8} 5 s^{2}$ & & $4 d^{8}\left({ }^{3} F\right) 5 s 5 p\left({ }^{3} P^{\circ}\right)$ & ${ }^{5} \mathrm{~F}_{4}^{\circ}$ & K02c & & & & \\
\hline 100000 & $2365.57671(17)$ & 42260.076 & $2365.57671(8)$ & 0.00001 & $4 d_{3 / 2}^{9} 5 p$ & ${ }^{2}[3 / 2]^{\circ}{ }_{1}$ & $4 d_{3 / 2}^{9} 5 d$ & ${ }^{2}[5 / 2]_{2}$ & K02c & $3.0 \mathrm{e}+08$ & $\mathrm{C}$ & TW & \\
\hline 100000 & 2363.88377(17) & 42290.339 & 2363.88381(10) & -0.00004 & $4 d^{9}{ }_{5 / 2}^{5 p}$ & ${ }^{2}[5 / 2]_{2}^{\circ}$ & $4 d^{9}{ }_{5 / 2} 5 d$ & ${ }^{2}[7 / 2]_{3}$ & K02c & $3.7 \mathrm{e}+08$ & C & TW & \\
\hline 67000 & $2362.07463(17)$ & 42322.727 & $2362.07464(9)$ & -0.00001 & $4 d^{9}{ }_{5 / 2} 5 p$ & ${ }^{2}[5 / 2]_{2}^{\circ}$ & $4 d^{9}{ }_{5 / 2} 5 d$ & ${ }^{2}[5 / 2]_{2}$ & K02c & $2.63 e+08$ & $\mathrm{~B}+$ & $\mathrm{C} 05$ & \\
\hline
\end{tabular}


Volume 118 (2013) http://dx.doi.org/10.6028/jres.118.009

Journal of Research of the National Institute of Standards and Technology

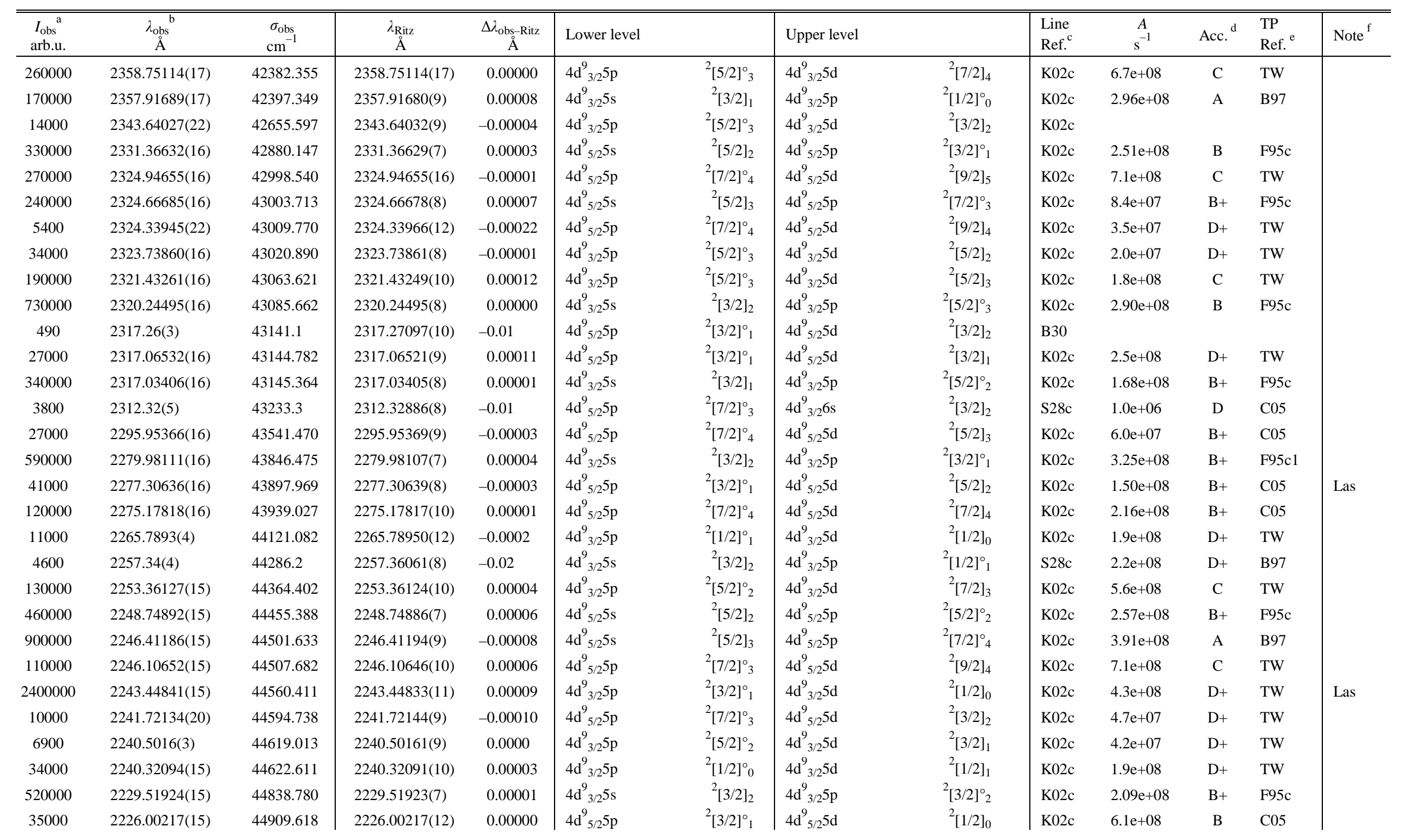


Volume 118 (2013) http://dx.doi.org/10.6028/jres.118.009

Journal of Research of the National Institute of Standards and Technology

\begin{tabular}{|c|c|c|c|c|c|c|c|c|c|c|c|c|c|}
\hline $\begin{array}{l}I_{\text {obs }}{ }^{\mathrm{a}} \\
\text { arb.u. }\end{array}$ & $\begin{array}{c}\lambda_{\text {obs }}{ }^{b} \\
\AA\end{array}$ & $\begin{array}{c}\sigma_{\mathrm{obs}} \\
\mathrm{cm}^{-1}\end{array}$ & $\stackrel{\lambda_{\text {Ritz }}}{\AA}$ & $\begin{array}{c}\Delta \lambda_{\text {obs-Ritz }} \\
\AA\end{array}$ & Lower level & & Upper leve & & $\begin{array}{l}\text { Line } \\
\text { Ref. }^{C}\end{array}$ & $\begin{array}{l}A \\
\mathrm{~s}^{-1}\end{array}$ & Acc. ${ }^{\mathrm{d}}$ & $\begin{array}{l}\text { TP } \\
\text { Ref. }^{\text {e }}\end{array}$ & Note $^{f}$ \\
\hline & & & 2224.00468(8) & & $4 d^{9}{ }_{5 / 2} 5 p$ & ${ }^{2}[3 / 2]^{\circ}{ }_{2}$ & $4 d^{9}{ }_{3 / 2} 6 \mathrm{~s}$ & ${ }^{2}[3 / 2]_{1}$ & & $4.0 \mathrm{e}+06$ & $\mathrm{D}$ & $\mathrm{C} 05$ & $\mathrm{P}$ \\
\hline 34000 & 2219.58790(15) & 45039.387 & 2219.58792(7) & -0.00002 & $4 d^{9}{ }_{5 / 2} 5 p$ & ${ }^{2}[7 / 2]^{\circ}{ }_{3}$ & $4 d^{9}{ }_{5 / 2} 5 d$ & ${ }^{2}[5 / 2]_{3}$ & K02c & $7.3 e+07$ & $\mathrm{~B}+$ & $\mathrm{C} 05$ & \\
\hline 9100 & 2218.7484(3) & 45056.426 & $2218.74835(15)$ & 0.0001 & $4 d^{9}{ }_{5 / 2} 5 p$ & ${ }^{2}[5 / 2]^{\circ}{ }_{3}$ & $4 d^{9}{ }_{3 / 2} 5 d$ & ${ }^{2}[7 / 2]_{4}$ & K02c & & & & \\
\hline 2000 & 2210.31012(24) & 45228.421 & $2210.31008(7)$ & 0.00003 & $4 d^{9}{ }_{5 / 2}^{5 p}$ & ${ }^{2}[3 / 2]_{2}^{\circ}$ & $4 d^{9}{ }_{3 / 2}^{6} 6 s$ & ${ }^{2}[3 / 2]_{2}$ & K02c & $3.00 \mathrm{e}+06$ & $\mathrm{~B}^{+}$ & $\mathrm{C} 05$ & \\
\hline 160000 & $2208.40105(15)$ & 45267.515 & $2208.40106(7)$ & -0.00001 & $4 d^{9}{ }_{3 / 2}^{5} p$ & ${ }^{2}[5 / 2]^{\circ}{ }_{2}$ & $4 d^{9}{ }_{3 / 2} 5 d$ & ${ }^{2}[5 / 2]_{2}$ & K02c & $1.9 \mathrm{e}+08$ & $\mathrm{D}^{+}$ & TW & \\
\hline 120000 & 2205.87431(15) & 45319.362 & 2205.87428(8) & 0.00003 & $4 d^{9}{ }_{5 / 2}^{5} p$ & ${ }^{2}[7 / 2]^{\circ}{ }_{3}$ & $4 d^{9}{ }_{5 / 2} 5 d$ & ${ }^{2}[7 / 2]_{3}$ & K02c & $2.5 \mathrm{e}+08$ & C & TW & \\
\hline 26000 & 2204.29872(19) & 45351.752 & 2204.29876(8) & -0.00004 & $4 d^{9}{ }_{5 / 2} 5 p$ & ${ }^{2}[7 / 2]^{\circ}{ }_{3}$ & $4 d^{9}{ }_{5 / 2} 5 d$ & ${ }^{2}[5 / 2]_{2}$ & K02c & $5.6 \mathrm{e}+07$ & $\mathrm{C}^{+}$ & $\mathrm{C} 05$ & \\
\hline 63000 & 2203.55651(15) & 45367.026 & 2203.55647(9) & 0.00003 & $4 d_{3 / 2}^{9} 5 p$ & ${ }^{2}[1 / 2]_{0}^{\circ}$ & $4 d^{9}{ }_{3 / 2} 5 d$ & ${ }^{2}[3 / 2]_{1}$ & K02c & $2.1 \mathrm{e}+08$ & $\mathrm{D}^{+}$ & TW & \\
\hline 120000 & 2202.03376(15) & 45398.395 & $2202.03378(11)$ & -0.00002 & $4 d^{9}{ }_{5 / 2}^{5 p}$ & ${ }^{2}[3 / 2]^{\circ}{ }_{2}$ & $4 d^{9}{ }_{5 / 2} 5 d$ & ${ }^{2}[1 / 2]_{1}$ & K02c & $6.7 \mathrm{e}+08$ & $\mathrm{C}$ & TW & \\
\hline & & & $2200.16534(11)$ & & $4 d^{9}{ }_{5 / 2} 5 p$ & ${ }^{2}[7 / 2]_{3}^{\circ}$ & $4 d^{9}{ }_{5 / 2} 5 d$ & ${ }^{2}[7 / 2]_{4}$ & & $3.0 \mathrm{e}+06$ & E & $\mathrm{C} 05$ & $\mathrm{P}$ \\
\hline 210000 & 2186.76813(14) & 45715.284 & 2186.76813(6) & 0.00000 & $4 d^{9}{ }_{5 / 2} 5 s$ & ${ }^{2}[5 / 2]_{2}$ & $4 d^{9}{ }_{5 / 2} 5 p$ & ${ }^{2}[5 / 2]^{\circ}{ }_{3}$ & K02c & $7.27 \mathrm{e}+07$ & A & F95c & \\
\hline 10000 & 2185.69653(19) & 45737.695 & 2185.69647(10) & 0.00005 & $4 d^{9}{ }_{5 / 2} 5 p$ & ${ }^{2}[5 / 2]^{\circ}{ }_{3}$ & $4 d^{9}{ }_{3 / 2} 5 d$ & ${ }^{2}[5 / 2]_{3}$ & K02c & & & & \\
\hline 28000 & 2171.6858(4) & 46032.743 & 2171.68597(8) & -0.0002 & $4 d^{9}{ }_{5 / 2} 5 s$ & ${ }^{2}[5 / 2]_{3}$ & $4 d^{9}{ }_{5 / 2} 5 p$ & ${ }^{2}[5 / 2]^{\circ}{ }_{2}$ & K02c & $3.9 \mathrm{e}+06$ & E & B97 & \\
\hline 84000 & $2170.78958(14)$ & 46051.745 & $2170.78956(10)$ & 0.00002 & $4 d^{9} 5 / 2 p$ & ${ }^{2}[5 / 2]^{\circ}{ }_{2}$ & $4 d_{3 / 2}^{9} 5 d$ & ${ }^{2}[7 / 2]_{3}$ & K02c & $1.6 \mathrm{e}+08$ & $\mathrm{C}$ & TW & \\
\hline 150000 & $2166.50867(14)$ & 46142.731 & $2166.50863(6)$ & 0.00004 & $4 d^{9} 5 / 25 s$ & ${ }^{2}[5 / 2]_{2}$ & $4 d^{9}{ }_{3 / 2} 5 p$ & ${ }^{2}[5 / 2]_{2}^{\circ}$ & K02c & $6.0 \mathrm{e}+07$ & B & F95c & \\
\hline 22000 & 2166.0359(3) & 46152.802 & 2166.03582(7) & 0.0000 & $4 d^{9}{ }_{3 / 2} 5 s$ & ${ }^{2}[3 / 2]_{1}$ & $4 d^{9}{ }_{3 / 2}^{5} p$ & ${ }^{2}[3 / 2]^{\circ}{ }_{1}$ & K02c & $1.7 \mathrm{e}+08$ & $\mathrm{D}^{+}$ & TW & \\
\hline 4700 & $2158.8525(4)$ & 46306.353 & 2158.85242(9) & 0.0001 & $4 d^{9}{ }_{5 / 2}^{5 p}$ & ${ }^{2}[5 / 2]^{\circ}{ }_{2}$ & $4 d_{3 / 2}^{9} 5 d$ & ${ }^{2}[3 / 2]_{1}$ & K02c & & & & \\
\hline 75000 & $2145.70735(14)$ & 46590.007 & 2145.70730(8) & 0.00005 & $4 d^{9}{ }_{5 / 2}^{5 p}$ & ${ }^{2}[3 / 2]_{2}^{\circ}$ & $4 d^{9}{ }_{5 / 2} 5 d$ & ${ }^{2}[3 / 2]_{2}$ & K02c & $5.2 \mathrm{e}+08$ & C & TW & \\
\hline 390000 & $2145.60920(14)$ & 46592.138 & $2145.60925(7)$ & -0.00006 & $4 d^{9}{ }_{3 / 2} 5 s$ & ${ }^{2}[3 / 2]_{1}$ & $4 d^{9}{ }_{3 / 2} 5 p$ & ${ }^{2}[1 / 2]^{\circ}{ }_{1}$ & K02c & $3.78 \mathrm{e}+08$ & $\mathrm{~B}^{+}$ & $\mathrm{F} 95 \mathrm{c} 1$ & \\
\hline 6000 & 2145.53095(18) & 46593.837 & 2145.53088(8) & 0.00007 & $4 d^{9} 5 / 2 p$ & ${ }^{2}[3 / 2]^{\circ}{ }_{2}$ & $4 d^{9}{ }_{5 / 2}^{5} \mathrm{~d}$ & ${ }^{2}[3 / 2]_{1}$ & K02c & $1.3 \mathrm{e}+08$ & $\mathrm{D}+$ & TW & \\
\hline 64000 & $2129.03277(14)$ & 46954.858 & $2129.03279(7)$ & -0.00002 & $4 d_{5 / 2}^{9} 5 p$ & ${ }^{2}[5 / 2]^{\circ}{ }_{2}$ & $4 d_{3 / 2}^{9} 5 d$ & ${ }^{2}[5 / 2]_{2}$ & K02c & $7.6 \mathrm{e}+07$ & $\mathrm{D}^{+}$ & TW & \\
\hline 66000 & $2125.42019(14)$ & 47034.658 & $2125.42016(7)$ & 0.00002 & $4 d^{9}{ }_{5 / 2}^{5 p}$ & ${ }^{2}[3 / 2]^{\circ}{ }_{2}$ & $4 d^{9}{ }_{5 / 2}^{5 d}$ & ${ }^{2}[5 / 2]_{3}$ & K02c & $1.47 \mathrm{e}+08$ & $\mathrm{~B}^{+}$ & $\mathrm{C} 05$ & \\
\hline 6900 & $2120.79683(18)$ & 47137.182 & $2120.79682(8)$ & 0.00001 & $4 d^{9}{ }_{5 / 2} 5 p$ & ${ }^{2}[3 / 2]^{\circ}{ }_{1}$ & $4 d_{3 / 2}^{9} 5 d$ & ${ }^{2}[1 / 2]_{1}$ & K02c & $1.5 \mathrm{e}+08$ & $\mathrm{D}^{+}$ & TW & \\
\hline 220000 & $2120.44020(13)$ & 47145.109 & $2120.44026(6)$ & -0.00007 & $4 d_{3 / 2}^{9} 5 s$ & ${ }^{2}[3 / 2]_{1}$ & $4 d_{3 / 2}^{9} 5 p$ & ${ }^{2}[3 / 2]_{2}^{\circ}$ & K02c & $1.19 \mathrm{e}+08$ & $\mathrm{~B}+$ & F95c & \\
\hline 530000 & 2113.82490(13) & 47292.634 & 2113.82492(7) & -0.00003 & $4 d^{9}{ }_{5 / 2} 5 s$ & ${ }^{2}[5 / 2]_{3}$ & $4 d^{9}{ }_{5 / 2} 5 p$ & ${ }^{2}[5 / 2]^{\circ}{ }_{3}$ & K02c & $3.01 \mathrm{e}+08$ & A & F95c & \\
\hline 4100 & 2111.40(3) & 47347.0 & 2111.39639(8) & 0.00 & $4 d^{9}{ }_{5 / 2} 5 p$ & ${ }^{2}[3 / 2]^{\circ}{ }_{2}$ & $4 d^{9}{ }_{5 / 2} 5 d$ & ${ }^{2}[5 / 2]_{2}$ & S28c & $1.00 \mathrm{e}+07$ & B & $\mathrm{C} 05$ & \\
\hline 140 & 2100.75(9) & 47587.0 & $2100.93065(13)$ & -0.18 & $4 d^{9}{ }_{5 / 2} 5 p$ & ${ }^{2}[7 / 2]^{\circ}{ }_{4}$ & $4 d^{9}{ }_{3 / 2} 5 d$ & ${ }^{2}[7 / 2]_{3}$ & G35c & & & & \\
\hline 800 & & & 2094.88826(7) & & $4 d^{9}{ }_{5 / 2} 5 \mathrm{~s}$ & ${ }^{2}[5 / 2]_{3}$ & $4 d^{9}{ }_{3 / 2} 5 p$ & ${ }^{2}[5 / 2]_{2}^{\circ}$ & F95 & $4.8 \mathrm{e}+05$ & $\mathrm{D}^{+}$ & F95c & $\mathrm{P}$ \\
\hline 740 & 2089.318(22) & 47847.3 & 2089.31191(13) & 0.006 & $4 d^{9}{ }_{5 / 2} 5 p$ & ${ }^{2}[7 / 2]^{\circ}{ }_{4}$ & $4 d_{3 / 2}^{9} 5 d$ & ${ }^{2}[7 / 2]_{4}$ & G35c & & & & \\
\hline 2300 & 2087.8208(4) & 47881.598 & 2087.82083(8) & 0.0000 & $4 d^{9} 5 / 2 p$ & ${ }^{2}[3 / 2]^{\circ}{ }_{1}$ & $4 d_{3 / 2}^{9} 5 d$ & ${ }^{2}[3 / 2]_{1}$ & K02c & & & & \\
\hline
\end{tabular}


Volume 118 (2013) http://dx.doi.org/10.6028/jres.118.009

Journal of Research of the National Institute of Standards and Technology

\begin{tabular}{|c|c|c|c|c|c|c|c|c|c|c|c|c|c|}
\hline $\begin{array}{c}I_{\text {obs }}^{\mathrm{a}} \\
\text { arb.u. }\end{array}$ & $\begin{array}{c}\lambda_{\text {obs }}{ }^{b} \\
\AA\end{array}$ & $\begin{array}{c}\sigma_{\mathrm{obs}} \\
\mathrm{cm}^{-1}\end{array}$ & $\begin{array}{c}\lambda_{\text {Ritz }} \\
\AA\end{array}$ & $\begin{array}{c}\Delta \lambda_{\text {obs-Ritz }} \\
\AA\end{array}$ & Lower lev & & Upper leve & & $\begin{array}{l}\text { Line } \\
\text { Ref. }^{C}\end{array}$ & $\begin{array}{l}A \\
\mathrm{~s}^{-1}\end{array}$ & Acc. ${ }^{\mathrm{d}}$ & $\begin{array}{l}\text { TP } \\
\text { Ref. }^{\text {e }}\end{array}$ & Note ${ }^{f}$ \\
\hline 36000 & 2075.54292(13) & 48164.806 & 2075.54294(7) & -0.00002 & $4 d^{9}{ }_{5 / 2} 5 p$ & ${ }^{2}[3 / 2]^{\circ}{ }_{1}$ & $4 d^{9}{ }_{3 / 2} 5 d$ & ${ }^{2}[3 / 2]_{2}$ & $\mathrm{~K} 02 \mathrm{c}$ & $1.2 \mathrm{e}+08$ & $\mathrm{D}+$ & TW & \\
\hline 120000 & 2065.91011(13) & 48389.357 & $2065.91012(6)$ & 0.00000 & $4 d^{9} 5 / 25 s$ & ${ }^{2}[5 / 2]_{2}$ & $4 d^{9}{ }_{3 / 2} 5 p$ & ${ }^{2}[5 / 2]^{\circ}{ }_{3}$ & $\mathrm{~K} 02 \mathrm{c}$ & $4.7 \mathrm{e}+07$ & $\mathrm{~B}^{+}$ & F95c & \\
\hline 540 & 2059.939(21) & 48529.6 & 2059.97746(10) & -0.038 & $4 d^{9} 5 / 25 p$ & ${ }^{2}[7 / 2]^{\circ}{ }_{4}$ & $4 d^{9}{ }_{3 / 2} 5 d$ & ${ }^{2}[5 / 2]_{3}$ & G35c & & & & \\
\hline 38000 & 2033.92680(12) & 49150.172 & 2033.92686(5) & -0.00006 & $4 d^{9} 5 / 25 s$ & ${ }^{2}[5 / 2]_{2}$ & $4 d^{9}{ }_{3 / 2}^{5 p}$ & ${ }^{2}[3 / 2]^{\circ}{ }_{1}$ & K02c & $2.4 \mathrm{e}+07$ & B & F95c1 & \\
\hline 570 & 2025.858(21) & 49345.9 & 2025.88091(12) & -0.023 & $4 d^{9}{ }_{5 / 2} 5 p$ & ${ }^{2}[7 / 2]^{\circ}{ }_{3}$ & $4 d^{9}{ }_{3 / 2} 5 d$ & ${ }^{2}[7 / 2]_{4}$ & G35c & & & & \\
\hline 34000 & 2015.90481(12) & 49589.507 & 2015.90491(6) & -0.00010 & $4 d^{9} 5 / 25 s$ & ${ }^{2}[5 / 2]_{2}$ & $4 d^{9}{ }_{3 / 2} 5 p$ & ${ }^{2}[1 / 2]^{\circ}{ }_{1}$ & K02c & $3.7 \mathrm{e}+07$ & $\mathrm{C}+$ & F95c1 & \\
\hline 40000 & 2000.68462(12) & 49966.703 & $2000.68449(6)$ & 0.00013 & $4 d^{9} 5 / 25 s$ & ${ }^{2}[5 / 2]_{3}$ & $4 d^{9}{ }_{3 / 2} 5 p$ & ${ }^{2}[5 / 2]_{3}^{\circ}$ & K02c & $1.75 \mathrm{e}+07$ & $\mathrm{~B}+$ & F95c & \\
\hline 730 & 1998.9350(8) & 50026.640 & 1998.93596(8) & -0.0010 & $4 d^{9} 5 / 2 p$ & ${ }^{2}[7 / 2]^{\circ}{ }_{3}$ & $4 d_{3 / 2}^{9} 5 d$ & ${ }^{2}[5 / 2]_{3}$ & K02c & & & & \\
\hline 140000 & $1994.31719(12)$ & 50142.475 & 1994.31719(5) & 0.00001 & $4 d^{9}{ }_{5 / 2} 5 s$ & ${ }^{2}[5 / 2]_{2}$ & $4 d_{3 / 2}^{9} 5 p$ & ${ }^{2}[3 / 2]_{2}^{\circ}$ & K02c & $4.08 \mathrm{e}+07$ & $\mathrm{~B}^{+}$ & F95c & \\
\hline 24000 & 1967.32521(12) & 50830.437 & 1967.32526(8) & -0.00005 & $4 d^{9} 5 / 2 p$ & ${ }^{2}[3 / 2]^{\circ}{ }_{1}$ & $4 d_{3 / 2}^{9} 5 d$ & ${ }^{2}[1 / 2]_{0}$ & K02c & & & & \\
\hline 260 & 1937.455(19) & 51614.1 & $1937.46408(7)$ & -0.009 & $4 d^{9}{ }_{5 / 2} 5 p$ & ${ }^{2}[3 / 2]_{2}^{\circ}$ & $4 d^{9}{ }_{3 / 2} 5 d$ & ${ }^{2}[3 / 2]_{2}$ & G35c & & & & \\
\hline 22000 & 1933.49455(11) & 51719.825 & 1933.49457(6) & -0.00001 & $4 d^{9}{ }_{5 / 2} 5 s$ & ${ }^{2}[5 / 2]_{3}$ & $4 d^{9}{ }_{3 / 2} 5 p$ & ${ }^{2}[3 / 2]^{\circ}{ }_{2}$ & K02c & $2.3 \mathrm{e}+07$ & $\mathrm{C}^{+}$ & F95c & \\
\hline 2200 & 1922.2672(3) & 52021.905 & 1922.26783(8) & -0.0007 & $4 d^{9}{ }_{5 / 2} 5 p$ & ${ }^{2}[3 / 2]_{2}^{\circ}$ & $4 d_{3 / 2}^{9} 5 d$ & ${ }^{2}[5 / 2]_{3}$ & K02c & & & & \\
\hline 180 & 1865.98(3) & 53591.2 & 1865.999(3) & -0.02 & $4 d^{9}{ }_{3 / 2} 5 p$ & ${ }^{2}[3 / 2]^{\circ}{ }_{2}$ & $4 d^{9}{ }_{5 / 2} 7 \mathrm{~s}$ & ${ }^{2}[5 / 2]_{3}$ & G35c & & & & \\
\hline 93 & $1860.88(10)$ & 53738 & $1860.9388(24)$ & -0.06 & $4 d^{9}{ }_{3 / 2} 5 p$ & ${ }^{2}[3 / 2]^{\circ}{ }_{2}$ & $4 d^{9} 5 / 27 \mathrm{~s}$ & ${ }^{2}[5 / 2]_{2}$ & G35c & & & & \\
\hline 210 & 1841.99(3) & 54289.0 & $1841.9840(24)$ & 0.01 & $4 d^{9}{ }_{3 / 2} 5 p$ & ${ }^{2}[1 / 2]^{\circ}{ }_{1}$ & $4 d^{9} 5 / 27 \mathrm{~s}$ & ${ }^{2}[5 / 2]_{2}$ & G35c & & & & \\
\hline 1100 & 1827.18(3) & 54729.1 & $1827.1975(23)$ & -0.02 & $4 d^{9}{ }_{3 / 2} 5 p$ & ${ }^{2}[3 / 2]^{\circ}{ }_{1}$ & $4 d^{9} 5 / 27 \mathrm{~s}$ & ${ }^{2}[5 / 2]_{2}$ & G35c & & & & \\
\hline 780 & 1806.87(3) & 55344.3 & 1806.890(3) & -0.02 & $4 d_{3 / 2}^{9} 5 p$ & ${ }^{2}[5 / 2]_{3}^{\circ}$ & $4 d^{9 / 2}{ }_{5 / 2} 7 \mathrm{~s}$ & ${ }^{2}[5 / 2]_{3}$ & G35c & & & & \\
\hline 1400 & 1802.19(3) & 55488.0 & $1802.1448(23)$ & 0.05 & $4 d^{9} 3 / 25 p$ & ${ }^{2}[5 / 2]^{\circ}{ }_{3}$ & $4 d^{9} 5 / 27 \mathrm{~s}$ & ${ }^{2}[5 / 2]_{2}$ & G35c & & & & \\
\hline 1900 & 1732.017(3) & 57736.16 & $1732.0198(21)$ & -0.003 & $4 d^{9} 3 / 25 p$ & ${ }^{2}[5 / 2]^{\circ}{ }_{2}$ & $4 d^{9} 5 / 27 \mathrm{~s}$ & ${ }^{2}[5 / 2]_{2}$ & K02c & & & & \\
\hline 5800 & 1723.612(3) & 58017.70 & 1723.609(3) & 0.003 & $4 d^{9} 5 / 2 p$ & ${ }^{2}[5 / 2]^{\circ}{ }_{3}$ & $4 d^{9} 5 / 27 \mathrm{~s}$ & ${ }^{2}[5 / 2]_{3}$ & K02c & $3.6 \mathrm{e}+07$ & $\mathrm{D}^{+}$ & TW & \\
\hline 2200 & 1719.295(3) & 58163.37 & $1719.2910(21)$ & 0.004 & $4 d^{9}{ }_{5 / 2}^{5 p}$ & ${ }^{2}[5 / 2]_{3}^{\circ}$ & $4 d_{5 / 2}^{9} 7 \mathrm{~s}$ & ${ }^{2}[5 / 2]_{2}$ & K02c & & & & \\
\hline 920 & $1718.360(3)$ & 58195.01 & $1718.360(3)$ & 0.000 & $4 d^{9} 3 / 25$ & ${ }^{2}[3 / 2]_{2}^{\circ}$ & $4 d^{9} 3 / 27 \mathrm{~s}$ & ${ }^{2}[3 / 2]_{1}$ & K02c & & & & \\
\hline 1500 & $1715.44(3)$ & 58294.1 & $1715.4325(24)$ & 0.01 & $4 d^{9}{ }_{3 / 2} 5 p$ & ${ }^{2}[3 / 2]_{2}^{\circ}$ & $4 \mathrm{~d}^{9}{ }_{3 / 2} 7 \mathrm{~s}$ & ${ }^{2}[3 / 2]_{2}$ & G35c & $3.4 \mathrm{e}+07$ & $\mathrm{D}^{+}$ & TW & \\
\hline 4000 & 1702.185(3) & 58748.02 & 1702.186(3) & -0.001 & $4 d^{9}{ }_{3 / 2} 5 p$ & ${ }^{2}[1 / 2]^{\circ}{ }_{1}$ & $4 \mathrm{~d}^{9}{ }_{3 / 2} 7 \mathrm{~s}$ & ${ }^{2}[3 / 2]_{1}$ & K02c & & & & \\
\hline 2200 & 1686.718(3) & 59286.75 & $1686.7206(23)$ & -0.003 & $4 d_{3 / 2}^{9} 5 p$ & ${ }^{2}[3 / 2]^{\circ}{ }_{1}$ & $4 d^{9}{ }_{3 / 2} 7 \mathrm{~s}$ & ${ }^{2}[3 / 2]_{2}$ & K02c & & & & \\
\hline 8000 & 1682.835(3) & 59423.52 & $1682.8386(20)$ & -0.003 & $4 d^{9} 5 / 2 p$ & ${ }^{2}[5 / 2]^{\circ}{ }_{2}$ & $4 d^{9} 5 / 27 \mathrm{~s}$ & ${ }^{2}[5 / 2]_{2}$ & K02c & $3.8 \mathrm{e}+07$ & $\mathrm{D}^{+}$ & TW & \\
\hline 14000 & 1665.351(3) & 60047.39 & $1665.3495(22)$ & 0.002 & $4 d^{9}{ }_{3 / 2} 5 p$ & ${ }^{2}[5 / 2]_{3}^{\circ}$ & $4 d^{9}{ }_{3 / 2} 7 \mathrm{~s}$ & ${ }^{2}[3 / 2]_{2}$ & K02c & $7.1 \mathrm{e}+07$ & $\mathrm{D}^{+}$ & TW & \\
\hline 7700 & 1644.496(3) & 60808.91 & $1644.4991(24)$ & -0.003 & $4 d^{9} 5 / 2 p$ & ${ }^{2}[7 / 2]^{\circ}{ }_{4}$ & $4 d^{9} 5 / 27 \mathrm{~s}$ & ${ }^{2}[5 / 2]_{3}$ & K02c & $7.1 \mathrm{e}^{+}+07$ & $\mathrm{D}^{+}$ & TW & \\
\hline 2400 & 1639.382(3) & 60998.59 & $1639.3807(19)$ & 0.001 & $4 d^{9} 5 / 2 p$ & ${ }^{2}[3 / 2]^{\circ}{ }_{1}$ & $4 d^{9} 5 / 27 \mathrm{~s}$ & ${ }^{2}[5 / 2]_{2}$ & K02c & & & & \\
\hline
\end{tabular}


Volume 118 (2013) http://dx.doi.org/10.6028/jres.118.009

Journal of Research of the National Institute of Standards and Technology

\begin{tabular}{|c|c|c|c|c|c|c|c|c|c|c|c|c|c|}
\hline $\begin{array}{l}I_{\text {obs }}{ }^{a} \\
\text { arb.u. }\end{array}$ & $\begin{array}{c}\lambda_{\text {obs }} \mathrm{b} \\
\AA \\
\AA\end{array}$ & $\begin{array}{c}\begin{array}{c}\sigma_{\mathrm{obs}} \\
\mathrm{cm}^{-1}\end{array} \\
\end{array}$ & $\begin{array}{c}\lambda_{\text {Ritz }} \\
\AA \\
\end{array}$ & $\begin{array}{c}\Delta \lambda_{\text {obs-Ritz }} \\
\AA\end{array}$ & Lower level & & Upper leve & & $\begin{array}{l}\text { Line } \\
\text { Ref. }^{\text {c }}\end{array}$ & $\begin{array}{l}A \\
\mathrm{~s}^{-1}\end{array}$ & Acc. $^{\mathrm{d}}$ & $\begin{array}{l}\text { TP } \\
\text { Ref. }^{\text {e }}\end{array}$ & Note $^{f}$ \\
\hline 3700 & 1607.88(3) & 62193.7 & $1607.8525(23)$ & 0.03 & $4 d^{9}{ }_{3 / 2} 5 p$ & ${ }^{2}[5 / 2]_{2}^{\circ}$ & $4 d^{9}{ }_{3 / 2} 7 \mathrm{~s}$ & ${ }^{2}[3 / 2]_{1}$ & G35c & $5.2 \mathrm{e}+07$ & $\mathrm{D}+$ & TW & \\
\hline 5000 & 1605.24(3) & 62296.0 & $1605.2890(21)$ & -0.05 & $4 d^{9}{ }_{3 / 2} 5 p$ & ${ }^{2}[5 / 2]^{\circ}{ }_{2}$ & $4 d^{9}{ }_{3 / 2} 7 \mathrm{~s}$ & ${ }^{2}[3 / 2]_{2}$ & G35c & & & & \\
\hline 3500 & 1604.96(3) & 62306.8 & $1604.9636(23)$ & 0.00 & $4 d^{9}{ }_{5 / 2} 5 p$ & ${ }^{2}[7 / 2]^{\circ}{ }_{3}$ & $4 d^{9} 5 / 27 \mathrm{~s}$ & ${ }^{2}[5 / 2]_{3}$ & G35c & & & & \\
\hline 7200 & 1601.24(3) & 62451.6 & $1601.2188(18)$ & 0.02 & $4 d^{9}{ }_{5 / 2} 5 p$ & ${ }^{2}[7 / 2]_{3}^{\circ}$ & $4 d^{9} 5 / 27 \mathrm{~s}$ & ${ }^{2}[5 / 2]_{2}$ & G35c & $6.3 \mathrm{e}+07$ & $\mathrm{D}^{+}$ & TW & \\
\hline 6300 & $1594.27(3)$ & 62724.5 & $1594.3489(20)$ & -0.08 & $4 d^{9}{ }_{5 / 2} 5 p$ & ${ }^{2}[5 / 2]^{\circ}{ }_{3}$ & $4 d^{9}{ }_{3 / 2} 7 \mathrm{~s}$ & ${ }^{2}[3 / 2]_{2}$ & G35c & & & & \\
\hline 3700 & 1588.77(3) & 62941.6 & $1588.7447(23)$ & 0.03 & $4 d^{9}{ }_{3 / 2} 5 p$ & ${ }^{2}[1 / 2]_{0}^{\circ}$ & $4 d^{9}{ }_{3 / 2} 7 \mathrm{~s}$ & ${ }^{2}[3 / 2]_{1}$ & G35c & & & & \\
\hline 1200 & $1565.376(25)$ & 63882.4 & $1565.3836(22)$ & -0.007 & $4 d^{9}{ }_{5 / 2} 5 p$ & ${ }^{2}[5 / 2]^{\circ}{ }_{2}$ & $4 d^{9} 3 / 27 \mathrm{~s}$ & ${ }^{2}[3 / 2]_{1}$ & G35c & & & & \\
\hline 8400 & 1555.188(24) & 64300.9 & $1555.1621(22)$ & 0.026 & $4 d^{9}{ }_{5 / 2}^{5 p}$ & ${ }^{2}[3 / 2]^{\circ}{ }_{2}$ & $4 d^{9} 5 / 27 \mathrm{~s}$ & ${ }^{2}[5 / 2]_{3}$ & G35c & $4.3 \mathrm{e}+07$ & $\mathrm{D}^{+}$ & TW & \\
\hline 420 & 1551.69(7) & 64446 & 1551.6458(17) & 0.04 & $4 d^{9}{ }_{5 / 2} 5 p$ & ${ }^{2}[3 / 2]_{2}^{\circ}$ & $4 d^{9} 5 / 27 \mathrm{~s}$ & ${ }^{2}[5 / 2]_{2}$ & G35c & & & & \\
\hline 860 & 1532.21(7) & 65265 & 1532.194(5) & 0.02 & $4 d_{3 / 2}^{9} 5 p$ & ${ }^{2}[3 / 2]^{\circ}{ }_{1}$ & $4 d^{9} 5 / 28 \mathrm{~s}$ & ${ }^{2}[5 / 2]_{2}$ & G35c & & & & \\
\hline 540 & 1527.74(7) & 65456 & $1527.7124(21)$ & 0.03 & $4 d^{9}{ }_{5 / 2} 5 p$ & ${ }^{2}[3 / 2]^{\circ}{ }_{1}$ & $4 d^{9}{ }_{3 / 2} 7 \mathrm{~s}$ & ${ }^{2}[3 / 2]_{1}$ & G35c & & & & \\
\hline 1100 & 1525.434(23) & 65555.1 & 1525.3979(19) & 0.036 & $4 d^{9}{ }_{5 / 2} 5 p$ & ${ }^{2}[3 / 2]^{\circ}{ }_{1}$ & $4 d^{9}{ }_{3 / 2} 7 \mathrm{~s}$ & ${ }^{2}[3 / 2]_{2}$ & G35c & & & & \\
\hline 800 & 1514.55(7) & 66026 & $1514.539(5)$ & 0.02 & $4 d_{3 / 2}^{9} 5 p$ & ${ }^{2}[5 / 2]^{\circ}{ }_{3}$ & $4 d^{9}{ }_{5 / 2} 8 \mathrm{~s}$ & ${ }^{2}[5 / 2]_{2}$ & G35c & & & & \\
\hline 5700 & $1492.268(22)$ & 67012.1 & $1492.3046(18)$ & -0.037 & $4 d^{9}{ }_{5 / 2} 5 p$ & ${ }^{2}[7 / 2]^{\circ}{ }_{3}$ & $4 d^{9}{ }_{3 / 2} 7 \mathrm{~s}$ & ${ }^{2}[3 / 2]_{2}$ & G35c & & & & \\
\hline 1300 & 1457.121(21) & 68628.5 & $1457.125(5)$ & -0.004 & $4 d^{9}{ }_{5 / 2} 5 p$ & ${ }^{2}[5 / 2]^{\circ}{ }_{3}$ & $4 d^{9} 5 / 28 \mathrm{~s}$ & ${ }^{2}[5 / 2]_{3}$ & G35c & & & & \\
\hline 660 & $1455.67(6)$ & 68697 & $1455.588(5)$ & 0.08 & $4 d^{9}{ }_{5 / 2}^{5} p$ & ${ }^{2}[5 / 2]_{3}^{\circ}$ & $4 d^{9} 5 / 28 \mathrm{~s}$ & ${ }^{2}[5 / 2]_{2}$ & G35c & & & & \\
\hline $900 ?$ & 1453.23(6) & 68812 & $1453.333(5)$ & -0.10 & $4 d_{3 / 2}^{9} 5 p$ & ${ }^{2}[3 / 2]^{\circ}{ }_{2}$ & $4 d^{9}{ }_{3 / 2} 8 \mathrm{~s}$ & ${ }^{2}[3 / 2]_{1}$ & G35c & & & & \\
\hline 420 & 1451.32(6) & 68903 & $1451.2440(19)$ & 0.07 & $4 d^{9}{ }_{5 / 2} 5 p$ & ${ }^{2}[3 / 2]^{\circ}{ }_{2}$ & $4 d^{9 / 2}{ }_{3 / 2}^{7 / s}$ & ${ }^{2}[3 / 2]_{1}$ & G35c & & & & \\
\hline 450 & 1449.1577(23) & 69005.60 & $1449.1552(17)$ & 0.0025 & $4 d^{9}{ }_{5 / 2} 5 p$ & ${ }^{2}[3 / 2]^{\circ}{ }_{2}$ & $4 d^{9} 3 / 27 \mathrm{~s}$ & ${ }^{2}[3 / 2]_{2}$ & K02c & & & & \\
\hline 900 & $1441.77(6)$ & 69359 & $1441.746(5)$ & 0.03 & $4 d_{3 / 2}^{9} 5 p$ & ${ }^{2}[1 / 2]^{\circ}{ }_{1}$ & $4 d^{9}{ }_{3 / 2} 8 \mathrm{~s}$ & ${ }^{2}[3 / 2]_{1}$ & G35c & & & & \\
\hline 910 & $1431.72(6)$ & 69846 & $1431.745(5)$ & -0.02 & $4 d_{3 / 2}^{9} 5 p$ & ${ }^{2}[3 / 2]^{\circ}{ }_{1}$ & $4 d^{9}{ }_{3 / 2} 8 \mathrm{~s}$ & ${ }^{2}[3 / 2]_{2}$ & G35c & & & & \\
\hline 650 & 1429.45(6) & 69957 & $1429.375(4)$ & 0.07 & $4 d^{9}{ }_{5 / 2}^{5 p}$ & ${ }^{2}[5 / 2]^{\circ}{ }_{2}$ & $4 d^{9}{ }_{5 / 2} 8 \mathrm{~s}$ & ${ }^{2}[5 / 2]_{2}$ & G35c & & & & \\
\hline 1900 & $1416.304(20)$ & 70606.3 & $1416.318(5)$ & -0.013 & $4 d^{9}{ }_{3 / 2}^{5 p}$ & ${ }^{2}[5 / 2]_{3}^{\circ}$ & $4 d^{9}{ }_{3 / 2} 8 \mathrm{~s}$ & ${ }^{2}[3 / 2]_{2}$ & G35c & & & & \\
\hline 2900 & $1400.194(20)$ & 71418.7 & $1400.182(5)$ & 0.012 & $4 d^{9}{ }_{5 / 2} 5 p$ & ${ }^{2}[7 / 2]^{\circ}{ }_{4}$ & $4 d^{9}{ }_{5 / 2} 8 \mathrm{~s}$ & ${ }^{2}[5 / 2]_{3}$ & G35c & $3.6 \mathrm{e}+07$ & $\mathrm{D}^{+}$ & TW & \\
\hline 750 & 1397.96(6) & 71533 & 1397.899(4) & 0.06 & $4 d^{9}{ }_{5 / 2} 5 p$ & ${ }^{2}[3 / 2]^{\circ}{ }_{1}$ & $4 d^{9}{ }_{5 / 2} 8 \mathrm{~s}$ & ${ }^{2}[5 / 2]_{2}$ & G35c & & & & \\
\hline 1400 & 1373.55(6) & 72804 & 1373.492(5) & 0.06 & $4 d_{3 / 2}^{9} 5 p$ & ${ }^{2}[5 / 2]_{2}^{\circ}$ & $4 d^{9}{ }_{3 / 2} 8 \mathrm{~s}$ & ${ }^{2}[3 / 2]_{1}$ & G35c & & & & \\
\hline 1400 & 1372.61(6) & 72854 & 1372.641(5) & -0.03 & $4 d^{9}{ }_{3 / 2} 5 p$ & ${ }^{2}[5 / 2]_{2}^{\circ}$ & $4 d^{9}{ }_{3 / 2} 8 \mathrm{~s}$ & ${ }^{2}[3 / 2]_{2}$ & G35c & & & & \\
\hline 1000 & $1371.48(6)$ & 72914 & $1371.418(4)$ & 0.06 & $4 d^{9}{ }_{5 / 2} 5 p$ & ${ }^{2}[7 / 2]_{3}^{\circ}$ & $4 d^{9}{ }_{5 / 2} 8 \mathrm{~s}$ & ${ }^{2}[5 / 2]_{3}$ & G35c & & & & \\
\hline 4200 & 1370.062(19) & 72989.4 & $1370.057(4)$ & 0.005 & $4 d^{9}{ }_{5 / 2}^{5} p$ & ${ }^{2}[7 / 2]_{3}^{\circ}$ & $4 d^{9} 5 / 28 \mathrm{~s}$ & ${ }^{2}[5 / 2]_{2}$ & G35c & & & & \\
\hline 1600 & $1364.65(6)$ & 73279 & $1364.634(4)$ & 0.01 & $4 d^{9}{ }_{5 / 2} 5 p$ & ${ }^{2}[5 / 2]^{\circ}{ }_{3}$ & $4 d^{9}{ }_{3 / 2} 8 \mathrm{~s}$ & ${ }^{2}[3 / 2]_{2}$ & G35c & & & & \\
\hline
\end{tabular}


Volume 118 (2013) http://dx.doi.org/10.6028/jres.118.009

Journal of Research of the National Institute of Standards and Technology

\begin{tabular}{|c|c|c|c|c|c|c|c|c|c|c|c|c|c|}
\hline $\begin{array}{l}I_{\text {obs }}{ }^{\mathrm{a}} \\
\text { arb.u. }\end{array}$ & $\begin{array}{c}\lambda_{\text {obs }}{ }^{b} \\
\AA\end{array}$ & $\begin{array}{l}\begin{array}{l}\sigma_{\mathrm{obs}} \\
\mathrm{cm}^{-1}\end{array}\end{array}$ & $\begin{array}{c}\lambda_{\text {Ritz }} \\
\AA\end{array}$ & $\begin{array}{c}\Delta \lambda_{\text {obs-Ritz }} \\
\AA\end{array}$ & Lower leve & & Upper level & & $\begin{array}{l}\text { Line } \\
\text { Ref. }^{c}\end{array}$ & $\begin{array}{l}A \\
\mathrm{~s}^{-1}\end{array}$ & Acc. ${ }^{d}$ & $\begin{array}{l}\text { TP } \\
\text { Ref. }^{\text {e }}\end{array}$ & Note $^{f}$ \\
\hline 2400 & 1334.92(5) & 74911 & 1334.891(4) & 0.03 & $4 d^{9}{ }_{5 / 2} 5 p$ & ${ }^{2}[3 / 2]^{\circ}{ }_{2}$ & $4 d^{9}{ }_{5 / 2} 8 \mathrm{~s}$ & ${ }^{2}[5 / 2]_{3}$ & G35c & & & & \\
\hline 2200000 & 1195.8091(11) & 83625.39 & 1195.8073(9) & 0.0018 & $4 d^{10}$ & ${ }^{1} \mathrm{~S}_{0}$ & $4 d^{9}{ }_{5 / 2} 5 p$ & ${ }^{2}[3 / 2]^{\circ}{ }_{1}$ & K02c & $5.7 \mathrm{e}+07$ & $\mathrm{D}^{+}$ & B97 & \\
\hline 39 & $1180.9712(22)$ & 84676.07 & $1180.9722(3)$ & -0.0010 & $4 d_{3 / 2}^{9} 5 \mathrm{~s}$ & ${ }^{2}[3 / 2]_{2}$ & $4 d^{8}\left({ }^{3} \mathrm{~F}\right) 5 \mathrm{~s} 5 \mathrm{p}\left({ }^{3} \mathrm{P}^{\circ}\right)$ & ${ }^{5} \mathrm{D}_{3}^{\circ}$ & K02c & & & & \\
\hline 160 & 1169.2079(10) & 85527.99 & 1169.2083(10) & -0.0004 & $4 d_{3 / 2}^{9} 5 s$ & ${ }^{2}[3 / 2]_{2}$ & $4 d^{9}{ }_{5 / 2} 6 p$ & ${ }^{2}[3 / 2]^{\circ}{ }_{2}$ & K02c & & & & \\
\hline 420 & 1163.2169(9) & 85968.49 & $1163.21641(20)$ & 0.0005 & $4 d_{3 / 2}^{9} 5 s$ & ${ }^{2}[3 / 2]_{2}$ & $4 d^{9}{ }_{5 / 2} 6 p$ & ${ }^{2}[7 / 2]_{3}^{\circ}$ & K02c & & & & \\
\hline 220 & 1152.164(13) & 86793.2 & 1152.17144(7) & -0.007 & $4 d_{3 / 2}^{9} 5 s$ & ${ }^{2}[3 / 2]_{2}$ & $4 d^{9}{ }_{5 / 2} 6 p$ & ${ }^{2}[3 / 2]^{\circ}{ }_{1}$ & G35c & & & & \\
\hline 290 & 1151.1284(9) & 86871.28 & $1151.12847(7)$ & 0.0000 & $4 d_{3 / 2}^{9} 5 s$ & ${ }^{2}[3 / 2]_{2}$ & $4 d^{9}{ }_{5 / 2} 6 p$ & ${ }^{2}[5 / 2]_{2}^{\circ}$ & K02c & & & & \\
\hline 250 & 1149.7824(9) & 86972.98 & $1149.78197(4)$ & 0.0004 & $4 d^{9}{ }_{3 / 2} 5 s$ & ${ }^{2}[3 / 2]_{2}$ & $4 d^{9}{ }_{5 / 2} 6 p$ & ${ }^{2}[5 / 2]_{3}^{\circ}$ & K02c & & & & \\
\hline 130 & 1148.2009(9) & 87092.77 & $1148.2007(4)$ & 0.0002 & $4 d^{9}{ }_{3 / 2} 5 s$ & ${ }^{2}[3 / 2]_{2}$ & $4 d^{8}\left({ }^{3} F\right) 5 s 5 p\left({ }^{3} P^{\circ}\right)$ & ${ }^{5} \mathrm{D}_{2}^{\circ}$ & K02c & & & & \\
\hline 65 & 1132.568(13) & 88294.9 & 1132.57885(9) & -0.011 & $4 d^{9}{ }_{3 / 2} 5 \mathrm{~s}$ & ${ }^{2}[3 / 2]_{2}$ & $4 d^{8}\left({ }^{3} F\right) 5 s 5 p\left({ }^{3} P^{\circ}\right)$ & ${ }^{5} \mathrm{G}_{3}^{\circ}$ & G35c & & & & \\
\hline 35 & 1126.76(5) & 88750 & 1126.75927(5) & 0.00 & $4 d_{3 / 2}^{9} 5 s$ & ${ }^{2}[3 / 2]_{2}$ & $4 d^{8}\left({ }^{3} F\right) 5 s 5 p\left({ }^{3} P^{\circ}\right)$ & ${ }^{5} \mathrm{D}^{\circ}{ }_{1}$ & G35c & & & & \\
\hline 84 & 1125.5403(9) & 88846.22 & $1125.54160(6)$ & -0.0013 & $4 d_{5 / 2}^{9} 5 s$ & ${ }^{2}[5 / 2]_{3}$ & $4 d^{8}\left({ }^{3} F\right) 5 s 5 p\left({ }^{3} P^{\circ}\right)$ & ${ }^{5} \mathrm{D}^{\circ}{ }_{4}$ & K02c & & & & \\
\hline 64 & 1122.339(13) & 89099.6 & $1122.34748(6)$ & -0.008 & $4 d_{3 / 2}^{9} 5 s$ & ${ }^{2}[3 / 2]_{1}$ & $4 d^{9}{ }_{5 / 2} 6 p$ & ${ }^{2}[3 / 2]^{\circ}{ }_{1}$ & G35c & & & & \\
\hline 120 & 1118.9895(9) & 89366.34 & 1118.99053(11) & -0.0010 & $4 d_{3 / 2}^{9} 5 s$ & ${ }^{2}[3 / 2]_{2}$ & $4 d^{8}\left({ }^{3} F\right) 5 s 5 p\left({ }^{3} P^{\circ}\right)$ & ${ }^{5} \mathrm{G}_{2}{ }_{2}$ & K02c & & & & \\
\hline 260 & 1118.5785(9) & 89399.18 & 1118.5793(4) & -0.0008 & $4 d_{3 / 2}^{9} 5 s$ & ${ }^{2}[3 / 2]_{1}$ & $4 d^{8}\left({ }^{3} F\right) 5 s 5 p\left({ }^{3} P^{\circ}\right)$ & ${ }^{5} \mathrm{D}_{2}^{\circ}$ & K02c & & & & \\
\hline 230000 & 1112.4005(7) & 89895.68 & 1112.4023(7) & -0.0018 & $4 d^{10}$ & ${ }^{1} \mathrm{~S}_{0}$ & $4 d_{3 / 2}^{9} 5 p$ & ${ }^{2}[3 / 2]^{\circ}{ }_{1}$ & K02c & $9 . e+08$ & $\mathrm{D}^{+}$ & TW & \\
\hline 50 & 1111.3619(9) & 89979.69 & 1111.3619(3) & 0.0000 & $4 d^{9}{ }_{5 / 2} 5 s$ & ${ }^{2}[5 / 2]_{2}$ & $4 d^{8}\left({ }^{3} \mathrm{~F}\right) 5 \mathrm{~s} 5 \mathrm{p}\left({ }^{3} \mathrm{P}^{\circ}\right)$ & ${ }^{5} \mathrm{D}_{3}^{\circ}$ & K02c & & & & \\
\hline 550000 & 1106.9930(7) & 90334.81 & 1106.9922(7) & 0.0008 & $4 d^{10}$ & ${ }^{1} \mathrm{~S}_{0}$ & $4 d_{3 / 2}^{9} 5 p$ & ${ }^{2}[1 / 2]^{\circ}{ }_{1}$ & K02c & $6.9 \mathrm{e}+08$ & $\mathrm{D}^{+}$ & B97 & \\
\hline 490 & 1105.3407(7) & 90469.84 & $1105.34083(21)$ & -0.0001 & $4 d_{3 / 2}^{9} 5 s$ & ${ }^{2}[3 / 2]_{2}$ & $4 d^{9}{ }_{3 / 2} 6 p$ & ${ }^{2}[5 / 2]_{2}^{\circ}$ & K02c & & & & \\
\hline 500 & 1101.5221(7) & 90783.47 & $1101.52218(17)$ & -0.0001 & $4 d_{3 / 2}^{9} 5 s$ & ${ }^{2}[3 / 2]_{2}$ & $4 d_{3 / 2}^{9} 6 p$ & ${ }^{2}[1 / 2]^{\circ}{ }_{1}$ & K02c & & & & \\
\hline 530 & 1098.242(12) & 91054.6 & 1098.22009(5) & 0.022 & $4 d_{3 / 2}^{9} 5 s$ & ${ }^{2}[3 / 2]_{1}$ & $4 d^{8}\left({ }^{3} F\right) 5 s 5 p\left({ }^{3} P^{\circ}\right)$ & ${ }^{5} \mathrm{D}_{1}^{\circ}$ & G35c & & & & \\
\hline 550 & 1097.3638(7) & 91127.48 & 1097.3641(3) & -0.0003 & $4 d^{9}{ }_{3 / 2} 5 s$ & ${ }^{2}[3 / 2]_{2}$ & $4 d^{9}{ }_{3 / 2} 6 p$ & ${ }^{2}[5 / 2]_{3}^{\circ}$ & K02c & & & & \\
\hline 150 & 1096.7758(7) & 91176.34 & 1096.77568(8) & 0.0001 & $4 d^{9}{ }_{3 / 2} 5 s$ & ${ }^{2}[3 / 2]_{2}$ & $4 d_{3 / 2}^{9} 6 p$ & ${ }^{2}[3 / 2]^{\circ}{ }_{1}$ & K02c & & & & \\
\hline 550 & 1095.6237(7) & 91272.21 & 1095.62361(17) & 0.0001 & $4 d^{9}{ }_{5 / 2} 5 s$ & ${ }^{2}[5 / 2]_{2}$ & $4 d^{9}{ }_{5 / 2} 6 p$ & ${ }^{2}[7 / 2]^{\circ}{ }_{3}$ & K02c & & & & \\
\hline 310 & 1093.8620(7) & 91419.21 & 1093.86220(12) & -0.0002 & $4 d^{9}{ }_{3 / 2} 5 \mathrm{~s}$ & ${ }^{2}[3 / 2]_{2}$ & $4 d^{9}{ }_{3 / 2} 6 p$ & ${ }^{2}[3 / 2]^{\circ} 2$ & K02c & & & & \\
\hline 460 & 1092.2152(7) & 91557.05 & 1092.2153(3) & -0.0001 & $4 d^{9}{ }_{5 / 2} 5 s$ & ${ }^{2}[5 / 2]_{3}$ & $4 d^{8}\left({ }^{3} F\right) 5 s 5 p\left({ }^{3} P^{\circ}\right)$ & ${ }^{5} \mathrm{D}_{3}^{\circ}$ & K02c & & & & \\
\hline 260 & $1090.8380(7)$ & 91672.64 & $1090.83862(11)$ & -0.0006 & $4 d_{3 / 2}^{9} 5 s$ & ${ }^{2}[3 / 2]_{1}$ & $4 d^{8}\left({ }^{3} F\right) 5 s 5 p\left({ }^{3} P^{\circ}\right)$ & ${ }^{5} \mathrm{G}_{2}{ }_{2}$ & K02c & & & & \\
\hline 410 & $1085.8184(7)$ & 92096.43 & 1085.81955(6) & -0.0011 & $4 d^{9}{ }_{5 / 2} 5 s$ & ${ }^{2}[5 / 2]_{2}$ & $4 d^{9}{ }_{5 / 2} 6 p$ & ${ }^{2}[3 / 2]^{\circ}{ }_{1}$ & K02c & & & & \\
\hline 670 & 1084.8911(7) & 92175.15 & 1084.89320(5) & -0.0021 & $4 d^{9}{ }_{5 / 2} 5 s$ & ${ }^{2}[5 / 2]_{2}$ & $4 d^{9}{ }_{5 / 2} 6 p$ & ${ }^{2}[5 / 2]^{\circ}{ }_{2}$ & K02c & & & & \\
\hline 550 & 1083.6971(7) & 92276.71 & $1083.69712(4)$ & 0.0000 & $4 d^{9}{ }_{5 / 2} 5 s$ & ${ }^{2}[5 / 2]_{2}$ & $4 d^{9}{ }_{5 / 2} 6 p$ & ${ }^{2}[5 / 2]^{\circ}{ }_{3}$ & K02c & & & & \\
\hline
\end{tabular}


Volume 118 (2013) http://dx.doi.org/10.6028/jres.118.009

Journal of Research of the National Institute of Standards and Technology

\begin{tabular}{|c|c|c|c|c|c|c|c|c|c|c|c|c|c|}
\hline $\begin{array}{c}I_{\mathrm{obs}}^{\mathrm{a}} \\
\text { arb.u. }\end{array}$ & $\begin{array}{c}\lambda_{\text {obs }}^{\mathrm{b}} \\
\AA \\
\end{array}$ & $\begin{array}{c}\begin{array}{c}\sigma_{\mathrm{obs}} \\
\mathrm{cm}^{-1}\end{array} \\
\end{array}$ & $\begin{array}{c}\lambda_{\text {Ritz }} \\
\AA\end{array}$ & $\begin{array}{c}\Delta \lambda_{\text {obs-Ritz }} \\
\AA\end{array}$ & Lower level & & Upper level & & $\begin{array}{l}\text { Line } \\
\text { Ref. }^{c}\end{array}$ & $\begin{array}{c}A \\
\mathrm{~s}^{-1}\end{array}$ & Acc. $^{\mathrm{d}}$ & $\begin{array}{l}\text { TP } \\
\text { Ref. }^{\text {e }}\end{array}$ & Note $^{f}$ \\
\hline 610 & 1082.2921(7) & 92396.50 & 1082.2923(4) & -0.0002 & $4 d^{9}{ }_{5 / 2} 5 s$ & ${ }^{2}[5 / 2]_{2}$ & $4 d^{8}\left({ }^{3} F\right) 5 s 5 p\left({ }^{3} P^{\circ}\right)$ & ${ }^{5} \mathrm{D}_{2}^{\circ}$ & K02c & & & & \\
\hline 820 & 1082.1458(7) & 92408.99 & 1082.1457(7) & 0.0001 & $4 d^{9} 5 / 25 s$ & ${ }^{2}[5 / 2]_{3}$ & $4 d^{9}{ }_{5 / 2} 6 p$ & ${ }^{2}[3 / 2]^{\circ}{ }_{2}$ & K02c & & & & \\
\hline 110 & 1080.09(5) & 92585 & $1080.0670(7)$ & 0.02 & $4 d^{9}{ }_{3 / 2} 5 \mathrm{~s}$ & ${ }^{2}[3 / 2]_{2}$ & $4 d^{8}\left({ }^{3} F\right) 5 s 5 p\left({ }^{3} P^{\circ}\right)$ & ${ }^{3} \mathrm{D}_{3}^{\circ}$ & G35c & & & & \\
\hline 1200 & 1077.8631(7) & 92776.16 & $1077.86310(20)$ & 0.0000 & $4 d^{9}{ }_{3 / 2} 5 \mathrm{~s}$ & ${ }^{2}[3 / 2]_{1}$ & $4 d^{9}{ }_{3 / 2} 6 \mathrm{p}$ & ${ }^{2}[5 / 2]^{\circ}{ }_{2}$ & K02c & & & & \\
\hline 740 & 1077.0106(7) & 92849.60 & 1077.01091(17) & -0.0003 & $4 d^{9}{ }_{5 / 2} 5 \mathrm{~s}$ & ${ }^{2}[5 / 2]_{3}$ & $4 d^{9}{ }_{5 / 2} 6 p$ & ${ }^{2}[7 / 2]^{\circ}{ }_{3}$ & K02c & & & & \\
\hline 1700 & $1072.2519(7)$ & 93261.67 & 1072.25210(3) & -0.0002 & $4 d^{9} 5 / 25 s$ & ${ }^{2}[5 / 2]_{3}$ & $4 d^{9}{ }_{5 / 2}^{6} 6 p$ & ${ }^{2}[7 / 2]^{\circ}{ }_{4}$ & K02c & & & & \\
\hline 1700 & 1069.7171(7) & 93482.66 & 1069.71694(8) & 0.0002 & $4 d^{9}{ }_{3 / 2} 5 s$ & ${ }^{2}[3 / 2]_{1}$ & $4 d^{9}{ }_{3 / 2}^{6} 6 p$ & ${ }^{2}[3 / 2]^{\circ}{ }_{1}$ & K02c & & & & \\
\hline 1000 & 1069.2823(7) & 93520.67 & 1069.28243(8) & -0.0001 & $4 d^{9}{ }_{5 / 2} 5 s$ & ${ }^{2}[5 / 2]_{3}$ & $4 d^{8}\left({ }^{3} F\right) 5 s 5 p\left({ }^{3} P^{\circ}\right)$ & ${ }^{5} \mathrm{G}^{\circ}{ }_{4}$ & K02c & & & & \\
\hline 1300 & 1068.4019(7) & 93597.74 & $1068.40155(8)$ & 0.0003 & $4 d^{9} 5 / 25 s$ & ${ }^{2}[5 / 2]_{2}$ & $4 d^{8}\left({ }^{3} \mathrm{~F}\right) 5 \mathrm{~s} 5 \mathrm{p}\left({ }^{3} \mathrm{P}^{\circ}\right)$ & ${ }^{5} \mathrm{G}_{3}^{\circ}$ & K02c & & & & \\
\hline 580 & 1066.9452(7) & 93725.53 & 1066.94527(11) & -0.0001 & $4 d^{9}{ }_{3 / 2} 5 s$ & ${ }^{2}[3 / 2]_{1}$ & $4 d_{3 / 2}^{9} 6 p$ & ${ }^{2}[3 / 2]_{2}^{\circ}$ & $\mathrm{K} 02 \mathrm{c}$ & & & & \\
\hline 210 & 1066.6403(7) & 93752.32 & 1066.64026(6) & 0.0000 & $4 d^{9}{ }_{5 / 2} 5 s$ & ${ }^{2}[5 / 2]_{3}$ & $4 d^{9}{ }_{5 / 2} 6 p$ & ${ }^{2}[5 / 2]_{2}^{\circ}$ & K02c & & & & \\
\hline 2100 & 1065.4841(7) & 93854.05 & 1065.48406(3) & 0.0001 & $4 d^{9}{ }_{5 / 2} 5 s$ & ${ }^{2}[5 / 2]_{3}$ & $4 d^{9}{ }_{5 / 2} 6 p$ & ${ }^{2}[5 / 2]_{3}^{\circ}$ & K02c & & & & \\
\hline 1100 & $1063.224(11)$ & 94053.6 & 1063.22130(5) & 0.002 & $4 d^{9}{ }_{5 / 2} 5 s$ & ${ }^{2}[5 / 2]_{2}$ & $4 d^{8}\left({ }^{3} F\right) 5 s 5 p\left({ }^{3} P^{\circ}\right)$ & ${ }^{5} \mathrm{D}^{\circ}{ }_{1}$ & G35c & & & & \\
\hline 920 & $1057.6448(7)$ & 94549.70 & $1057.6457(7)$ & -0.0009 & $4 d^{9}{ }_{3 / 2} 5 \mathrm{~s}$ & ${ }^{2}[3 / 2]_{2}$ & $4 d^{8}\left({ }^{3} F\right) 5 s 5 p\left({ }^{3} P^{\circ}\right)$ & ${ }^{3} \mathrm{G}_{3}^{\circ}$ & K02c & & & & \\
\hline 2500 & $1056.3027(6)$ & 94669.83 & $1056.30133(10)$ & 0.0014 & $4 d^{9} 5 / 25 s$ & ${ }^{2}[5 / 2]_{2}$ & $4 d^{8}\left({ }^{3} \mathrm{~F}\right) 5 \mathrm{~s} 5 \mathrm{p}\left({ }^{3} \mathrm{P}^{\circ}\right)$ & ${ }^{5} \mathrm{G}_{2}^{\circ}$ & K02c & & & & \\
\hline 2300 & $1050.6948(6)$ & 95175.12 & $1050.69479(8)$ & 0.0000 & $4 d^{9} 5 / 25 s$ & ${ }^{2}[5 / 2]_{3}$ & $4 d^{8}\left({ }^{3} F\right) 5 s 5 p\left({ }^{3} \mathrm{P}^{\circ}\right)$ & ${ }^{5} \mathrm{G}_{3}^{\circ}$ & K02c & & & & \\
\hline 2900 & $1047.2603(5)$ & 95487.24 & 1047.2600(6) & 0.0004 & $4 d^{9}{ }_{3 / 2} 5 s$ & ${ }^{2}[3 / 2]_{1}$ & $4 d^{8}\left({ }^{3} F\right) 5 s 5 p\left({ }^{3} P^{\circ}\right)$ & ${ }^{3} \mathrm{D}_{2}^{\circ}$ & $\mathrm{K} 02 \mathrm{c}$ & & & & \\
\hline 340 & 1044.1292(8) & 95773.59 & $1044.12986(19)$ & -0.0007 & $4 d^{9} 5 / 25 s$ & ${ }^{2}[5 / 2]_{2}$ & $4 d^{9}{ }_{3 / 2} 6 \mathrm{p}$ & ${ }^{2}[5 / 2]^{\circ}{ }_{2}$ & K02c & & & & \\
\hline 3400 & $1043.0659(5)$ & 95871.22 & $1043.0655(5)$ & 0.0004 & $4 d^{9}{ }_{3 / 2} 5 \mathrm{~s}$ & ${ }^{2}[3 / 2]_{2}$ & $4 d^{8}\left({ }^{3} F\right) 5 s 5 p\left({ }^{3} P^{\circ}\right)$ & ${ }^{3} \mathrm{~F}_{3}^{\circ}$ & K02c & & & & \\
\hline 3100 & $1040.7217(5)$ & 96087.17 & $1040.72178(15)$ & -0.0001 & $4 d^{9}{ }_{5 / 2} 5 s$ & ${ }^{2}[5 / 2]_{2}$ & $4 d_{3 / 2}^{9} 6 p$ & ${ }^{2}[1 / 2]^{\circ}{ }_{1}$ & K02c & & & & \\
\hline 910 & 1038.9900(5) & 96247.32 & 1038.99013(10) & -0.0002 & $4 d^{9}{ }_{5 / 2} 5 s$ & ${ }^{2}[5 / 2]_{3}$ & $4 d^{8}\left({ }^{3} \mathrm{~F}\right) 5 \mathrm{~s} 5 \mathrm{p}\left({ }^{3} \mathrm{P}^{\circ}\right)$ & ${ }^{5} \mathrm{G}_{2}^{\circ}$ & K02c & & & & \\
\hline 600 & $1038.4537(12)$ & 96297.02 & $1038.4540(6)$ & -0.0002 & $4 d_{3 / 2}^{9} 5 s$ & ${ }^{2}[3 / 2]_{2}$ & $4 d^{8}\left({ }^{3} F\right) 5 s 5 p\left({ }^{3} P^{\circ}\right)$ & ${ }^{3} \mathrm{D}_{1}^{\circ}$ & K02c & & & & \\
\hline 6300 & 1037.0110(5) & 96430.99 & $1037.0093(3)$ & 0.0018 & $4 d^{9}{ }_{5 / 2} 5 \mathrm{~s}$ & ${ }^{2}[5 / 2]_{2}$ & $4 d_{3 / 2}^{9} 6 p$ & ${ }^{2}[5 / 2]^{\circ}{ }_{3}$ & K02c & & & & \\
\hline 1600 & $1036.4837(5)$ & 96480.05 & 1036.48380(8) & -0.0001 & $4 d_{5 / 2}^{9} 5 s$ & ${ }^{2}[5 / 2]_{2}$ & $4 d_{3 / 2}^{9} 6 p$ & ${ }^{2}[3 / 2]_{1}^{\circ}$ & K02c & & & & \\
\hline 8800 & $1034.2345(5)$ & 96689.87 & 1034.23469(13) & -0.0002 & $4 d^{9}{ }_{5 / 2} 5 s$ & ${ }^{2}[5 / 2]_{2}$ & $4 d^{8}\left({ }^{3} F\right) 5 s 5 p\left({ }^{3} P^{\circ}\right)$ & & $\mathrm{K} 02 \mathrm{c}$ & & & & \\
\hline 4600 & 1033.8811(5) & 96722.92 & $1033.88145(11)$ & -0.0003 & $4 d^{9}{ }_{5 / 2} 5 s$ & ${ }^{2}[5 / 2]_{2}$ & $4 d_{3 / 2}^{9} 6 \mathrm{p}$ & ${ }^{2}[3 / 2]_{2}^{\circ}$ & K02c & & & & \\
\hline 4500 & 1031.5741(5) & 96939.23 & $1031.57619(10)$ & -0.0021 & $4 d^{9} 5 / 25 s$ & ${ }^{2}[5 / 2]_{3}$ & $4 d^{8}\left({ }^{3} F\right) 5 s 5 p\left({ }^{3} P^{\circ}\right)$ & ${ }^{5} \mathrm{~F}_{4}^{\circ}$ & K02c & & & & \\
\hline 6300 & $1028.5770(5)$ & 97221.70 & $1028.5769(5)$ & 0.0001 & $4 d^{9}{ }_{3 / 2} 5 \mathrm{~s}$ & ${ }^{2}[3 / 2]_{1}$ & $4 d^{8}\left({ }^{3} \mathrm{P}\right) 5 \mathrm{~s} 5 \mathrm{p}\left({ }^{3} \mathrm{P}^{\circ}\right)$ & ${ }^{5} \mathrm{P}_{2}^{\circ}$ & K02c & & & & \\
\hline 14000 & $1028.3243(5)$ & 97245.59 & $1028.3239(5)$ & 0.0004 & $4 d_{3 / 2}^{9} 5 \mathrm{~s}$ & ${ }^{2}[3 / 2]_{1}$ & $4 d^{8}\left({ }^{3} \mathrm{P}\right) 5 \mathrm{~s} 5 \mathrm{p}\left({ }^{3} \mathrm{P}^{\circ}\right)$ & ${ }^{5} \mathrm{P}^{\circ}{ }_{1}$ & K02c & & & & \\
\hline 12000 & 1021.5493(5) & 97890.53 & 1021.5491(5) & 0.0001 & $4 d_{5 / 2}^{9} 5 s$ & ${ }^{2}[5 / 2]_{2}$ & $4 d^{8}\left({ }^{3} \mathrm{~F}\right) 5 \mathrm{~s} 5 \mathrm{p}\left({ }^{3} \mathrm{P}^{\circ}\right)$ & ${ }^{3} \mathrm{D}_{3}^{\circ}$ & K02c & $1.2 \mathrm{e}+08$ & $\mathrm{D}^{+}$ & TW & \\
\hline
\end{tabular}


Volume 118 (2013) http://dx.doi.org/10.6028/jres.118.009

Journal of Research of the National Institute of Standards and Technology

\begin{tabular}{|c|c|c|c|c|c|c|c|c|c|c|c|c|c|}
\hline $\begin{array}{l}I_{\mathrm{obs}}{ }^{\mathrm{a}} \\
\text { arb.u. }\end{array}$ & $\begin{array}{c}\lambda_{\text {obs }}{ }^{b} \\
\AA\end{array}$ & $\begin{array}{l}\begin{array}{l}\sigma_{\mathrm{obs}} \\
\mathrm{cm}^{-1}\end{array}\end{array}$ & $\begin{array}{c}\lambda_{\text {Ritz }} \\
\AA\end{array}$ & $\underset{\AA}{\Delta \lambda_{\text {obs-Ritz }}}$ & Lower leve & & Upper level & & $\begin{array}{l}\text { Line } \\
\text { Ref. }^{c}\end{array}$ & $\begin{array}{c}A \\
\mathrm{~s}^{-1}\end{array}$ & Acc. $^{\mathrm{d}}$ & $\begin{array}{l}\text { TP } \\
\text { Ref. }^{\text {e }}\end{array}$ & Note $^{f}$ \\
\hline 3300 & $1020.3195(5)$ & 98008.52 & $1020.31964(25)$ & -0.0002 & $4 d^{9}{ }_{5 / 2} 5 s$ & ${ }^{2}[5 / 2]_{3}$ & $4 d_{3 / 2}^{9} 6 p$ & ${ }^{2}[5 / 2]^{\circ}{ }_{3}$ & K02c & & & & \\
\hline 1900 & $1017.2918(6)$ & 98300.21 & $1017.29154(10)$ & 0.0003 & $4 d^{9}{ }_{5 / 2} 5 s$ & ${ }^{2}[5 / 2]_{3}$ & $4 d_{3 / 2}^{9} 6 p$ & ${ }^{2}[3 / 2]^{\circ}{ }_{2}$ & K02c & & & & \\
\hline 15000 & $1015.3869(5)$ & 98484.63 & 1015.3867(5) & 0.0001 & $4 d^{9} 5 / 25 s$ & ${ }^{2}[5 / 2]_{2}$ & $4 d^{8}\left({ }^{3} F\right) 5 s 5 p\left({ }^{3} P^{\circ}\right)$ & ${ }^{3} \mathrm{D}_{2}^{\circ}$ & K02c & $1.5 \mathrm{e}+08$ & $\mathrm{D}^{+}$ & TW & \\
\hline 27000 & 1014.1648(5) & 98603.30 & $1014.1646(5)$ & 0.0003 & $4 d^{9}{ }_{3 / 2} 5 \mathrm{~s}$ & ${ }^{2}[3 / 2]_{1}$ & $4 d^{8}\left({ }^{3} F\right) 5 s 5 p\left({ }^{3} P^{\circ}\right)$ & ${ }^{3} \mathrm{D}^{\circ}{ }_{1}$ & K02c & $3.0 \mathrm{e}+08$ & $\mathrm{D}^{+}$ & TW & \\
\hline 36000 & $1010.2435(5)$ & 98986.04 & $1010.24336(7)$ & 0.0001 & $4 d_{3 / 2}^{9} 5 \mathrm{~s}$ & ${ }^{2}[3 / 2]_{2}$ & $4 d^{8}\left({ }^{3} \mathrm{~F}\right) 5 \mathrm{~s} 5 \mathrm{p}\left({ }^{3} \mathrm{P}^{\circ}\right)$ & ${ }^{1} \mathrm{D}_{2}^{\circ}$ & K02c & $6.1 \mathrm{e}+08$ & $\mathrm{D}^{+}$ & TW & \\
\hline 10000 & 1008.5859(6) & 99148.72 & $1008.58622(4)$ & -0.0003 & $4 d_{3 / 2}^{9} 5 \mathrm{~s}$ & ${ }^{2}[3 / 2]_{2}$ & $4 d_{5 / 2}^{9} 4 f$ & ${ }^{2}[3 / 2]^{\circ}{ }_{2}$ & K02c & $2.1 \mathrm{e}+08$ & $\mathrm{D}^{+}$ & TW & \\
\hline 33000 & 1008.1275(5) & 99193.80 & 1008.12768(5) & -0.0002 & $4 d_{3 / 2}^{9} 5 s$ & ${ }^{2}[3 / 2]_{2}$ & $4 d^{9} 5 / 24 f$ & ${ }^{2}[3 / 2]^{\circ}{ }_{1}$ & K02c & & & & \\
\hline 51000 & 1007.4503(5) & 99260.48 & $1007.45044(6)$ & -0.0001 & $4 d_{3 / 2}^{9} 5 s$ & ${ }^{2}[3 / 2]_{2}$ & $4 d^{9}{ }_{5 / 2} 4 f$ & ${ }^{2}[5 / 2]^{\circ}{ }_{2}$ & K02c & & & & \\
\hline 7800 & $1006.4487(6)$ & 99359.26 & $1006.44894(3)$ & -0.0002 & $4 d^{9}{ }_{3 / 2} 5 s$ & ${ }^{2}[3 / 2]_{2}$ & $4 d^{9}{ }_{5 / 2} 4 f$ & ${ }^{2}[7 / 2]^{\circ}{ }_{3}$ & K02c & & & & \\
\hline 48000 & $1005.3493(5)$ & 99467.92 & 1005.3495(5) & -0.0003 & $4 d^{9}{ }_{5 / 2} 5 s$ & ${ }^{2}[5 / 2]_{3}$ & $4 d^{8}\left({ }^{3} F\right) 5 s 5 p\left({ }^{3} P^{\circ}\right)$ & ${ }^{3} \mathrm{D}_{3}^{\circ}$ & K02c & $3.8 \mathrm{e}+08$ & $\mathrm{D}^{+}$ & TW & \\
\hline 42000 & 1004.9292(5) & 99509.50 & $1004.92924(6)$ & -0.0001 & $4 d^{9}{ }_{3 / 2} 5 s$ & ${ }^{2}[3 / 2]_{2}$ & $4 d^{8}\left({ }^{3} F\right) 5 s 5 p\left({ }^{3} P^{\circ}\right)$ & ${ }^{1} \mathrm{~F}_{3}^{\circ}$ & K02c & $1.0 \mathrm{e}+08$ & $\mathrm{D}^{+}$ & TW & \\
\hline 23000 & 1001.4694(5) & 99853.28 & $1001.4690(5)$ & 0.0003 & $4 d^{9} 5 / 25 s$ & ${ }^{2}[5 / 2]_{2}$ & $4 d^{8}\left({ }^{3} F\right) 5 s 5 p\left({ }^{3} P^{\circ}\right)$ & ${ }^{3} \mathrm{G}_{3}^{\circ}$ & K02c & & & & \\
\hline 26000 & $999.3800(5)$ & 100062.04 & 999.3805(5) & -0.0005 & $4 d^{9} 5 / 25 s$ & ${ }^{2}[5 / 2]_{3}$ & $4 d^{8}\left({ }^{3} F\right) 5 s 5 p\left({ }^{3} P^{\circ}\right)$ & ${ }^{3} \mathrm{D}_{2}^{\circ}$ & K02c & & & & \\
\hline 4700 & $999.2586(10)$ & 100074.20 & $999.2587(7)$ & -0.0001 & $4 d^{9}{ }_{3 / 2} 5 \mathrm{~s}$ & ${ }^{2}[3 / 2]_{2}$ & $4 d^{8}\left({ }^{1} D\right) 5 s 5 p\left({ }^{3} \mathrm{P}^{\circ}\right)$ & ${ }^{3} \mathrm{~F}_{2}^{\circ}$ & K02c & & & & \\
\hline 42000 & $997.8142(5)$ & 100219.06 & $997.8141(5)$ & 0.0001 & $4 d^{9}{ }_{5 / 2} 5 \mathrm{~s}$ & ${ }^{2}[5 / 2]_{2}$ & $4 d^{8}\left({ }^{3} \mathrm{P}\right) 5 \mathrm{~s} 5 \mathrm{p}\left({ }^{3} \mathrm{P}^{\circ}\right)$ & ${ }^{5} \mathrm{P}_{2}^{\circ}$ & K02c & $3.1 \mathrm{e}+08$ & $\mathrm{D}^{+}$ & TW & \\
\hline 18000 & $997.5756(5)$ & 100243.03 & $997.5760(5)$ & -0.0004 & $4 d^{9} 5 / 25 s$ & ${ }^{2}[5 / 2]_{2}$ & $4 d^{8}\left({ }^{3} P\right) 5 s 5 p\left({ }^{3} P^{\circ}\right)$ & ${ }^{5} \mathrm{P}^{\circ}{ }_{1}$ & K02c & & & & \\
\hline 8500 & 995.9494(6) & 100406.71 & $995.9490(6)$ & 0.0003 & $4 d_{5 / 2}^{9} 5 \mathrm{~s}$ & ${ }^{2}[5 / 2]_{2}$ & $4 d^{8}\left({ }^{3} \mathrm{P}\right) 5 \mathrm{~s} 5 \mathrm{p}\left({ }^{3} \mathrm{P}^{\circ}\right)$ & ${ }^{5} \mathrm{P}_{3}^{\circ}$ & K02c & & & & \\
\hline 43000 & 988.3865(5) & 101175.00 & 988.3869(5) & -0.0005 & $4 d_{5 / 2}^{9} 5 \mathrm{~s}$ & ${ }^{2}[5 / 2]_{2}$ & $4 d^{8}\left({ }^{3} F\right) 5 s 5 p\left({ }^{3} P^{\circ}\right)$ & ${ }^{3} \mathrm{~F}_{3}^{\circ}$ & K02c & $2.0 \mathrm{e}+08$ & $\mathrm{D}^{+}$ & TW & \\
\hline 58000 & 985.8954(5) & 101430.64 & 985.8952(5) & 0.0002 & $4 d^{9}{ }_{5 / 2} 5 s$ & ${ }^{2}[5 / 2]_{3}$ & $4 d^{8}\left({ }^{3} \mathrm{~F}\right) 5 \mathrm{~s} 5 \mathrm{p}\left({ }^{3} \mathrm{P}^{\circ}\right)$ & ${ }^{3} \mathrm{G}_{3}^{\circ}$ & K02c & $3.1 \mathrm{e}+08$ & $\mathrm{D}^{+}$ & TW & \\
\hline 2600 & 985.2196(17) & 101500.21 & $985.22059(5)$ & -0.0009 & $4 d_{3 / 2}^{9} 5 s$ & ${ }^{2}[3 / 2]_{1}$ & $4 d^{9}{ }_{5 / 2} 4 f$ & ${ }^{2}[3 / 2]^{\circ}{ }_{1}$ & K02c & & & & \\
\hline 14000 & $984.2449(6)$ & 101600.73 & $984.2453(6)$ & -0.0004 & $4 d^{9}{ }_{5 / 2} 5 s$ & ${ }^{2}[5 / 2]_{2}$ & $4 d^{8}\left({ }^{3} F\right) 5 s 5 p\left({ }^{3} P^{\circ}\right)$ & ${ }^{3} \mathrm{D}^{\circ}{ }_{1}$ & K02c & & & & \\
\hline 18000 & 982.3525(5) & 101796.45 & $982.3528(5)$ & -0.0003 & $4 d^{9}{ }_{5 / 2} 5 s$ & ${ }^{2}[5 / 2]_{3}$ & $4 d^{8}\left({ }^{3} \mathrm{P}\right) 5 \mathrm{~s} 5 \mathrm{p}\left({ }^{3} \mathrm{P}^{\circ}\right)$ & ${ }^{5} \mathrm{P}_{2}^{\circ}$ & K02c & & & & \\
\hline 58000 & $980.5448(5)$ & 101984.12 & $980.5451(5)$ & -0.0003 & $4 d^{9}{ }_{5 / 2}^{5 s}$ & ${ }^{2}[5 / 2]_{3}$ & $4 d^{8}\left({ }^{3} \mathrm{P}\right) 5 \mathrm{~s} 5 \mathrm{p}\left({ }^{3} \mathrm{P}^{\circ}\right)$ & ${ }^{5} \mathrm{P}_{3}^{\circ}$ & K02c & & & & \\
\hline 76000 & 976.7483(5) & 102380.52 & $976.7484(5)$ & 0.0000 & $4 d^{9}{ }_{3 / 2} 5 s$ & ${ }^{2}[3 / 2]_{1}$ & $4 d^{8}\left({ }^{1} \mathrm{D}\right) 5 \mathrm{~s} 5 \mathrm{p}\left({ }^{3} \mathrm{P}^{\circ}\right)$ & ${ }^{3} \mathrm{~F}_{2}^{\circ}$ & K02c & $2.1 \mathrm{e}+08$ & $\mathrm{D}^{+}$ & TW & \\
\hline 26000 & $973.2142(5)$ & 102752.30 & $973.2142(5)$ & 0.0000 & $4 d^{9} 5 / 25 s$ & ${ }^{2}[5 / 2]_{3}$ & $4 d^{8}\left({ }^{3} F\right) 5 s 5 p\left({ }^{3} P^{\circ}\right)$ & ${ }^{3} \mathrm{~F}_{3}^{\circ}$ & K02c & $4.4 \mathrm{e}+08$ & $\mathrm{D}^{+}$ & TW & \\
\hline 70000 & $970.8875(5)$ & 102998.54 & $970.8876(5)$ & -0.0001 & $4 d^{9} 5 / 25 s$ & ${ }^{2}[5 / 2]_{2}$ & $4 d^{8}\left({ }^{3} F\right) 5 s 5 p\left({ }^{3} P^{\circ}\right)$ & ${ }^{3} \mathrm{~F}_{2}^{\circ}$ & K02c & $7.3 e+08$ & $\mathrm{D}^{+}$ & TW & \\
\hline 4500 & 958.8663(9) & 104289.83 & $958.86705(6)$ & -0.0008 & $4 d^{9}{ }_{5 / 2} 5 s$ & ${ }^{2}[5 / 2]_{2}$ & $4 d^{8}\left({ }^{3} F\right) 5 s 5 p\left({ }^{3} P^{\circ}\right)$ & ${ }^{1} \mathrm{D}_{2}^{\circ}$ & K02c & $2.2 \mathrm{e}+08$ & $\mathrm{D}^{+}$ & TW & \\
\hline 5100 & $957.3740(8)$ & 104452.39 & $957.37404(4)$ & -0.0001 & $4 d_{5 / 2}^{9} 5 s$ & ${ }^{2}[5 / 2]_{2}$ & $4 d^{9}{ }_{5 / 2} 4 f$ & ${ }^{2}[3 / 2]_{2}^{\circ}$ & K02c & & & & \\
\hline 18000 & 956.9606(5) & 104497.51 & $956.96088(5)$ & -0.0003 & $4 d_{5 / 2}^{9} 5 s$ & ${ }^{2}[5 / 2]_{2}$ & $4 d^{9}{ }_{5 / 2} 4 \mathrm{f}$ & ${ }^{2}[3 / 2]^{\circ}{ }_{1}$ & K02c & & & & \\
\hline 18000 & $956.3500(5)$ & 104564.23 & 956.35062(5) & -0.0006 & $4 d_{5 / 2}^{9} 5 s$ & ${ }^{2}[5 / 2]_{2}$ & $4 d^{9}{ }_{5 / 2} 4 f$ & ${ }^{2}[5 / 2]^{\circ}{ }_{2}$ & K02c & & & & \\
\hline
\end{tabular}


Volume 118 (2013) http://dx.doi.org/10.6028/jres.118.009

Journal of Research of the National Institute of Standards and Technology

\begin{tabular}{|c|c|c|c|c|c|c|c|c|c|c|c|c|c|}
\hline $\begin{array}{l}I_{\mathrm{obs}}^{\mathrm{a}} \\
\text { arb.u. }\end{array}$ & $\begin{array}{c}\lambda_{\text {obs }}{ }^{b} \\
\AA\end{array}$ & $\begin{array}{c}\sigma_{\mathrm{obs}} \\
\mathrm{cm}^{-1}\end{array}$ & $\begin{array}{c}\lambda_{\text {Ritz }} \\
\AA\end{array}$ & $\begin{array}{c}\Delta \lambda_{\text {obs-Ritz }} \\
\AA\end{array}$ & Lower level & & Upper level & & $\begin{array}{l}\text { Line } \\
\text { Ref. }^{c}\end{array}$ & $\begin{array}{l}A \\
\mathrm{~s}^{-1}\end{array}$ & Acc. ${ }^{\mathrm{d}}$ & $\begin{array}{l}\text { TP } \\
\text { Ref. }^{\text {e }}\end{array}$ & Note $^{f}$ \\
\hline 14000 & 956.2436(5) & 104575.86 & 956.2434(5) & 0.0002 & $4 d^{9} 5 / 25$ & ${ }^{2}[5 / 2]_{3}$ & $4 d^{8}\left({ }^{3} F\right) 5 s 5 p\left({ }^{3} P^{\circ}\right)$ & ${ }^{3} \mathrm{~F}_{2}^{\circ}$ & K02c & $1.5 \mathrm{e}+08$ & $\mathrm{D}^{+}$ & TW & \\
\hline 35000 & $954.0780(5)$ & 104813.23 & $954.07840(5)$ & -0.0004 & $4 d^{9}{ }_{5 / 2} 5 s$ & ${ }^{2}[5 / 2]_{2}$ & $4 d^{8}\left({ }^{3} F\right) 5 s 5 p\left({ }^{3} P^{\circ}\right)$ & ${ }^{1} \mathrm{~F}_{3}^{\circ}$ & K02c & & & & \\
\hline 3500 & $948.9659(10)$ & 105377.86 & $948.9657(6)$ & 0.0002 & $4 d^{9} 5 / 25 s$ & ${ }^{2}[5 / 2]_{2}$ & $4 d^{8}\left({ }^{1} D\right) 5 s 5 p\left({ }^{3} P^{\circ}\right)$ & ${ }^{3} \mathrm{~F}_{2}^{\circ}$ & K02c & & & & \\
\hline 11000 & $943.1323(5)$ & 106029.66 & 943.13168(4) & 0.0006 & $4 d^{9} 5 / 25$ & ${ }^{2}[5 / 2]_{3}$ & $4 d^{9}{ }_{5 / 2} 4 f$ & ${ }^{2}[3 / 2]_{2}^{\circ}$ & K02c & & & & \\
\hline 34000 & 752.793(11) & 132838.6 & $752.7757(3)$ & 0.018 & $4 d^{10}$ & ${ }^{1} \mathrm{~S}_{0}$ & $4 d^{9}{ }_{5 / 2}^{6} 6 p$ & ${ }^{2}[3 / 2]^{\circ}{ }_{1}$ & G35c & & & & \\
\hline 15000 & $730.816(11)$ & 136833.3 & $730.8204(4)$ & -0.004 & $4 d^{10}$ & ${ }^{1} \mathrm{~S}_{0}$ & $4 d_{3 / 2}^{9} 6 p$ & ${ }^{2}[1 / 2]^{\circ}{ }_{1}$ & G35c & & & & \\
\hline 1600 & $728.717(11)$ & 137227.4 & $728.7280(4)$ & -0.011 & $4 \mathrm{~d}^{10}$ & ${ }^{1} \mathrm{~S}_{0}$ & $4 d^{9}{ }_{3 / 2}^{6} 6 p$ & ${ }^{2}[3 / 2]^{\circ}{ }_{1}$ & G35c & & & & \\
\hline
\end{tabular}

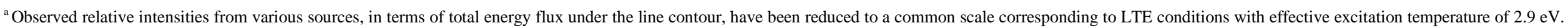
Variations of sensitivity of registration equipment with wavelength have been removed from intensity values (see text). Where several observations were available, they have been averaged.

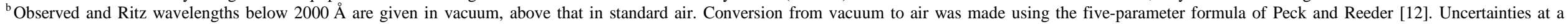
confidence level of one standard deviation are given in parentheses after the value in units of the last decimal place of the value.

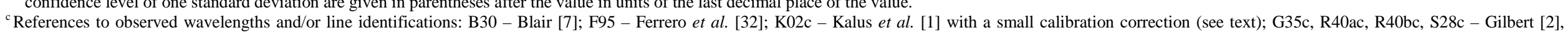
Rasmussen [3,4], and Shenstone [6], respectively, recalibrated using internal wave number standards based on FTS measurements of Kalus et al. [1].

${ }^{\mathrm{d}}$ Accuracy codes for $A$-values are explained in Table 3.

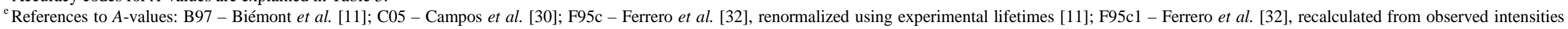
using the Boltzmann equation, Eq. (4); TW - present work.

${ }^{\mathrm{f}}$ Notes: Las - Lasing was observed on these lines in hollow-cathode discharges [21,22]; P - predicted (not observed); $\mathrm{S}$ - this line alone determines one of the levels involved in the transition. 


\subsection{Observations of Kalus et al. [1]}

As mentioned in the Introduction, the light source used in Kalus et al. [1] was a pulsed hollow cathode discharge. Argon, neon, and helium mixtures were used as carrier gases. Neon and helium were used as it was found by many authors that collisional transfer of charge and energy from $\mathrm{He}^{+}$and $\mathrm{Ne}^{+}$greatly enhances lines from highly excited levels of $\mathrm{Ag}^{+}$. Two different measurement techniques were used in two wavelength regions. Measurements of 97 lines in the vacuum ultraviolet (VUV) region below $1800 \AA$ were made on photographic recordings of spectra obtained with a $10.7 \mathrm{~m}$ vacuum grating spectrograph. Above $1800 \AA$, the spectrum was recorded with a Fourier transform spectrometer (FTS); 216 lines were measured in this spectral region.

The wavelength scale of the FTS measurements was calibrated by means of Ar II lines measured by Norlén [13]. Uncertainties of the FTS lines, including the calibration uncertainty, ranged from $0.003 \mathrm{~cm}^{-1}$ for strong lines to $0.03 \mathrm{~cm}^{-1}$ for the weakest lines.

For the VUV spectrum, the wavelength scale was initially calibrated using internal standards consisting of Ne II lines from the carrier gas and C I, N II, and O II impurity lines, as well as external Cu II standards provided by an auxiliary Cu-Ne hollow cathode. The final calibration included a large number of Ag II Ritz wavelengths of 5s-6p lines, derived from the energy levels established from the FTS measurements. Uncertainties of the VUV lines were in the range from $0.0005 \AA$ for strong lines to $0.002 \AA$ for weak lines.

Nave and Sansonetti [14] have found that the wave number scale of Norlén [13] has a calibration error, and all wave numbers from his paper have to be increased by 6.7 parts in $10^{8}$. Since all measurements of Kalus et al. [1] were ultimately based on the scale of Norlén, I applied the above correction factor to all their wave numbers. The correction amounted to $0.0008 \mathrm{~cm}^{-1}$ to $0.0035 \mathrm{~cm}^{-1}$ for the FTS measurements and $0.004 \mathrm{~cm}^{-1}$ to $0.007 \mathrm{~cm}^{-1}$ for the VUV grating measurements. While for the VUV region the correction is well below the stated measurement uncertainties, $(7-12) \times 10^{-5} \AA$ for wavelengths, the correction of the FTS measurements is significant, especially for short wavelengths.

To estimate the wave number uncertainties, I assumed that the measurement uncertainties $\delta \sigma$ are entirely due to statistical and systematic uncertainties ( $\delta \sigma_{\text {stat }}$ and $\delta \sigma_{\text {syst }}$, respectively) in the wave number measurements of symmetric well-resolved features (see, for example, Kramida and Nave [15]):

$$
\begin{gathered}
\delta \sigma \approx\left(\delta{\sigma_{\text {stat }}}^{2}+\delta \sigma_{\text {syst }}^{2}\right)^{1 / 2}, \\
\delta \sigma_{\text {stat }} \approx W /(2 S / N),
\end{gathered}
$$

where $W$ is the full width at half-maximum. The signal-to-noise ratios $S / N$ are given in the line list of Kalus et al. [1] in the column of line intensities. The values of $W$ and $\delta \sigma_{\text {syst }}$ can be estimated as $0.03 \mathrm{~cm}^{-1}$ and $0.003 \mathrm{~cm}^{-1}$, respectively, from the statement above about the uncertainties of the FTS measurements quoted from [1]. Uncertainties of the VUV grating measurements given in Table 1 were estimated using similar considerations.

To verify the assigned uncertainties, I calculated the energy levels with the least-squares level optimization code LOPT [16] using only the uncorrected wave numbers listed by Kalus et al. [1] with the uncertainties assigned as described above. The resulting energy level values were in close agreement with those given by Kalus et al., which confirms that the assigned uncertainties are close to those used by Kalus et al. in their level optimization.

In Table 1, the observed wavelengths referred to Kalus et al. [1] were obtained from the wave numbers, corrected as described above.

\subsection{Observations of Rasmussen [3,4]}

Rasmussen observed the Ag II spectrum between $2530 \AA$ and $9053 \AA$ with a hollow cathode of pure silver in neon [3,4] and helium [4] discharges. The first paper [3] is a preliminary report of the more extensive studies reported in [4]. 
Rasmussen specified the uncertainty of his wavelength measurements to be about $0.01 \AA$. He listed the wavelengths rounded off to two places after the decimal point. However, his listed wave numbers are given with higher precision. Comparison of these wave numbers with the Ritz values based mainly on the FTS measurements of Kalus et al. [1] reveals that they have a relative statistical uncertainty $\Delta \sigma / \sigma=2.0 \times 10^{-6}$ and a systematic shift smoothly varying from +1 part in $10^{6}$ at $18000 \mathrm{~cm}^{-1}$ to +4 parts in $10^{6}$ at $33000 \mathrm{~cm}^{-1}$ (see Fig. 1).

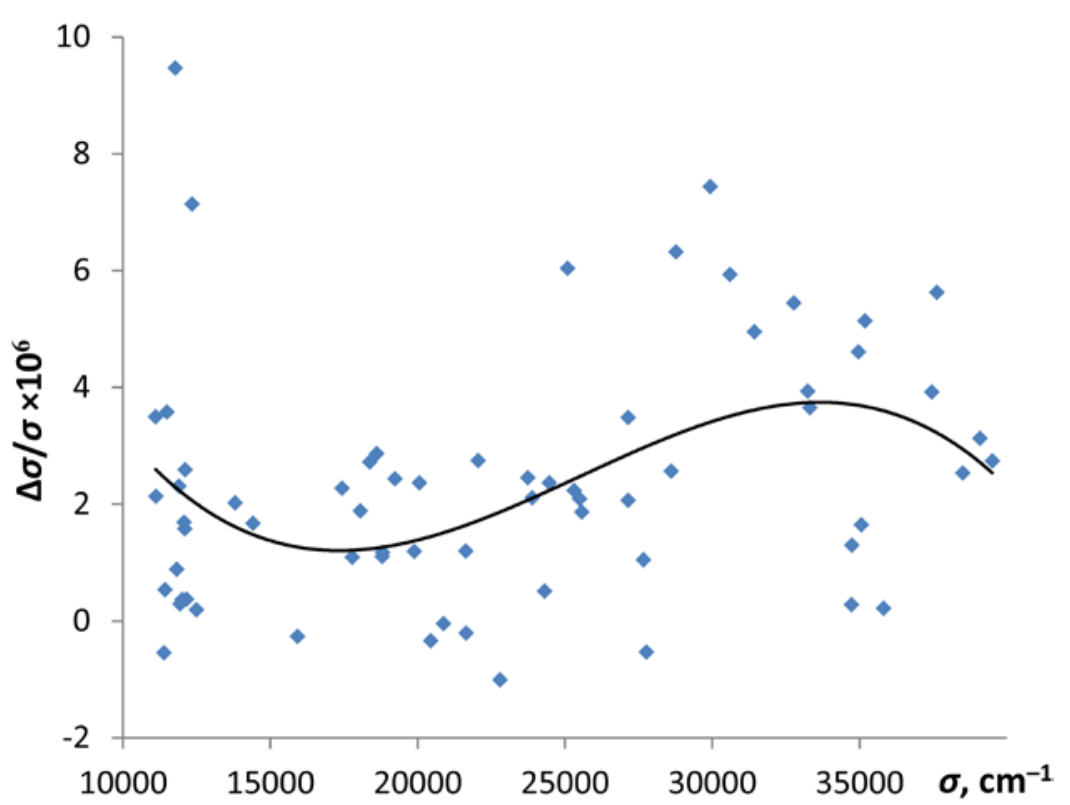

Fig. 1. Relative deviations of wave numbers measured by Rasmussen [4] from Ritz wave numbers based on measurements of Kalus $e t$ al. [1]. The smooth curve is a cubic polynomial that fits the data points.

I removed this systematic shift by subtracting the values represented by the smooth curve shown in Fig. 1 from the wave numbers of Rasmussen. The statistical uncertainties of the resulting wavelengths vary from $\pm 0.005 \AA$ at $2530 \AA$ to $\pm 0.018 \AA$ at $9000 \AA$.

\subsection{Observations of Gilbert [2]}

In the work of Gilbert [2], the spectrum was excited in a pulsed hollow cathode discharge with a lowpressure helium carrier gas. Enhancement of lines originating from highly excited levels was achieved by using a spark gap in series with the hollow cathode. Wavelength measurements were made in the region from $728 \AA$ to $2600 \AA$ with a 1.5-meter grating vacuum spectrograph and in the region from $4000 \AA$ to $11000 \AA$ with a 3-prism spectrograph. Impurity lines of oxygen, nitrogen, carbon, helium, and hydrogen, as well as Ag II lines previously measured by Shenstone [6] were used as internal standards in the region below $2600 \AA$. The measurements were believed to be accurate to $0.05 \AA$ in this region $\left(1.4 \mathrm{~cm}^{-1}\right.$ at $1900 \AA$ to $5 \mathrm{~cm}^{-1}$ at $1000 \AA$ ). In the region above $4000 \AA$, the standards were furnished by an iron comparison spectrum. The measurement uncertainty was estimated as $\pm 1 \mathrm{~cm}^{-1}$ over this region $(0.16 \AA$ at $4100 \AA$ and $1.0 \AA$ at $9000 \AA)$.

As in the case of the data of Rasmussen, the measurements of Kalus et al. [1] now provide an excellent set of internal references. Re-calibration of the wave numbers reported by Gilbert against the Ritz wave numbers derived from the measurements of Kalus et al. results in significant improvement of accuracy. The corrected wave numbers and wavelengths obtained from them are given in Table 1 . For unblended lines with large or medium intensity, uncertainties are $2 \mathrm{~cm}^{-1}$ below $1000 \AA(0.011 \AA$ at $728 \AA$ to $0.02 \AA$ at 
$1000 \AA$ ), $1.0 \mathrm{~cm}^{-1}$ in the region $1000 \AA$ to $1900 \AA(0.010 \AA$ at $1000 \AA$ to $0.03 \AA$ at $1900 \AA), 0.5 \mathrm{~cm}^{-1}$ in the region $1937 \AA$ to $2186 \AA$ (about $0.02 \AA$ ), $0.2 \mathrm{~cm}^{-1}$ in the region $4313 \AA$ to $4531 \AA$ (about $0.04 \AA$ ), and $0.4 \mathrm{~cm}^{-1}$ above $6279 \AA(0.2 \AA$ at $6279 \AA$ to $0.3 \AA$ at $9000 \AA)$. For weak or unresolved lines, uncertainties are much greater, about $3 \mathrm{~cm}^{-1}(0.04 \AA$ at $1080 \AA$ and $2.3 \AA$ at $9000 \AA)$. The correction applied to wave numbers measured by Gilbert [2] varies between $-3.4 \mathrm{~cm}^{-1}$ and $+2.3 \mathrm{~cm}^{-1}$, being the greatest at $1370 \AA$.

Three lines listed by Gilbert with wave numbers $94429.6 \mathrm{~cm}^{-1}, 89847.3 \mathrm{~cm}^{-1}$, and $21499.7 \mathrm{~cm}^{-1}$ were classified as transitions to or from the odd-parity level designated by Gilbert as $3_{2}$, located at $133589.4 \mathrm{~cm}^{-1}$. Since my energy-level calculations (see Sec. 4) show that this level is not real, and since these three lines cannot be classified as transitions between any known levels, I discarded the spurious level and the lines.

\subsection{Observations of Blair [7]}

Blair [7] photographed the Ag II spectrum in the region $2000 \AA$ to $3400 \AA$ emitted by a hollow cathode. Only 20 newly classified lines were given in this paper. Most of them were remeasured by Kalus et al. [1] with much greater accuracy. However, seven lines were not reported elsewhere. To determine their uncertainties, I compared wavelengths reported by Blair with the Ritz wavelengths derived from the measurements of Kalus et al. [1]. From this comparison, it follows that the measurements of Blair [7] have a statistical uncertainty of $0.04 \AA$ with a small systematic shift of about $+0.01 \AA$. Since this shift is much smaller than the statistical uncertainty, and all the levels involved are precisely determined by the measurements of Kalus et al. [1], I did not remove this shift from the wavelengths reported by Blair.

\subsection{The Work of Shenstone [6]}

All observations reported in the work of Shenstone [6] were made with a spark source. For wavelengths above 2246 A, Shenstone listed measurements of Exner and Haschek [17], with few exceptions where he gave his own measured values or those from Frings [18]. Most of the lines reported by Shenstone were accurately re-measured by Kalus et al. [1]. However, there are 12 lines not reported by other observers, 10 quoted from [17] and two measured by Shenstone. These lines are between $2111 \AA$ and $3270 \AA$. As in the previous cases, it was possible to re-calibrate the wavelength scales of Shenstone and of Exner and Haschek by using Ritz wavelengths based on the measurements of Kalus et al. [1] as internal standards. This procedure revealed the presence of large systematic shifts, which are nearly the same in the measurements of Shenstone and of Exner and Haschek and amount to $+0.04 \AA$ at $2111 \AA$ and $+0.25 \AA$ at $3270 \AA$. After removing this shift, the resulting wavelengths reported in Table 1 have statistical uncertainties ranging from $0.03 \AA$ at $2111 \AA$ to $0.05 \AA$ at $3270 \AA$. There is one exception for the weak unresolved line at $2829.0 \AA$, which deviates from the (much more accurate) Ritz wavelength by $0.4 \AA$ $\left(5 \mathrm{~cm}^{-1}\right)$. For this line, I adopted the uncertainty of $0.4 \AA$.

\subsection{The Work of Benschop et al. [5]}

Benschop et al. [5] observed the VUV spectrum of singly and multiply ionized silver in the region $500 \AA$ to $2200 \AA$ by using two light sources: 1) a sliding spark with a ceramic or quartz spacer, and 2) a triggered vacuum spark. Three high-resolution vacuum grating spectrographs facilitated wavelength measurements with uncertainties about or below $0.005 \AA$. Cu II lines excited in a copper hollow cathode lamp in a helium atmosphere were used as standards in the region above $700 \AA$. Between $500 \AA$ and $700 \AA$, either $\mathrm{O}, \mathrm{C}$, and $\mathrm{N}$ lines or some silver lines measured in the higher orders were used as internal standards. The total number of observed spectral lines was over 10000. Most of these lines were found to be due to $\mathrm{Ag}$ III-V $[19,20]$. Separation of lines belonging to different ionization stages was achieved by varying the operational conditions of the discharges.

Benschop et al. [5] stated that they re-measured many of the previously known Ag II lines with greater accuracy and confirmed the previous analysis. However, they listed only 64 lines that were newly classified in their work and gave revised values of 74 energy levels. Thirty-two of the new lines were classified as 
transitions between levels that are now known with high accuracy from Refs. [1,3-4]. Only one of these lines (1313.809 $\AA, 4 \mathrm{~d}^{9} 5 \mathrm{p}^{3} \mathrm{P}^{\circ}{ }_{1}-4 \mathrm{~d}^{9} 8 \mathrm{~s}^{1} \mathrm{D}_{2}$ ) agrees with the Ritz wavelength (1313.804(4) $\AA$ ) within the combined uncertainties. The root-mean-square (rms) deviation of the measured wavelengths from the Ritz values is $0.09 \AA$, i.e., 18 times the claimed measurement uncertainty. The other 32 lines ascribed by Benschop et al. to the previously unknown $4 \mathrm{~d}^{9} 9 \mathrm{~s}$ configuration and $4 \mathrm{~d}^{8} 5 \mathrm{~s}^{2}{ }^{1} \mathrm{~S}_{0}$ level have similarly large deviations of wavelengths from the Ritz values. These deviations appear to be random and have both large positive and large negative values. They do not show any regular trend that could be interpreted as a smoothly varying calibration error. The rms deviation of the energy level values obtained by Benschop et al. from the new, much more accurate values (see Table 2 and Sec. 3) is about $6 \mathrm{~cm}^{-1}$, while the uncertainty implied by their wavelength measurements should not exceed $0.5 \mathrm{~cm}^{-1}$. The values of $4 \mathrm{~d}^{9} 6 \mathrm{p}^{3} \mathrm{P}^{\circ}{ }_{2}$ and $4 \mathrm{~d}^{9} 5 \mathrm{~d}^{3} \mathrm{D}_{2}$ deviate from those of Kalus et al. by $21 \mathrm{~cm}^{-1}$ and $-42 \mathrm{~cm}^{-1}$, respectively. In addition, the observed relative intensities of the lines have no correlation with calculated radiative rates (see Sec. 6). Therefore, the entire analysis of Benschop et al. [5] must be deemed incorrect, and their results must be disregarded, including the identification of the missing ${ }^{1} \mathrm{~S}_{0}$ level of the $4 \mathrm{~d}^{8} 5 \mathrm{~s}^{2}$ configuration, the $4 d^{9} 9$ s levels, and their derivation of the ionization energy.

A special note should be made about the line at $1160.887 \AA$ assigned to the $4 \mathrm{~d}^{10}{ }^{1} \mathrm{~S}_{0}-4 \mathrm{~d}^{9} 5 \mathrm{p}^{3} \mathrm{P}^{\circ}{ }_{2}$ transition [5]. Its wave number does not agree with the energy of the $4 \mathrm{~d}^{9} 5 \mathrm{p}^{3} \mathrm{P}^{\circ}{ }_{2}$ level given by Benschop et al. [5]. The closest match is with the $4 \mathrm{~d}^{9} 5 \mathrm{p}^{3} \mathrm{P}^{\circ}{ }_{0}$ level, which differs from the wave number of this line by $5 \mathrm{~cm}^{-1}$. Neither classification is feasible, since all $4 \mathrm{~d}^{9} 5 \mathrm{p}$ levels have allowed (electric-dipole) radiative transitions to lower even-parity levels of the $4 \mathrm{~d}^{9} 5 \mathrm{~s}$ configuration. Forbidden transitions from these levels must have very small branching ratios.

\subsection{Other Observations}

Several lines of Ag II in the range $2243 \AA$ to $8404 \AA$ were found by Reid et al. [21,22] to exhibit lasing in hollow cathode discharges filled with Ne or He. Eleven lasing lines were identified with known Ag II transitions listed in Refs. [1,2]. Several unidentified lines reported in other papers of the same team $[23,24]$ were found by Reid et al. [21] to be diffraction-grating ghost lines. The identified lasing lines are marked in Table 1 with a corresponding note.

Tables of the Massachusetts Institute of Technology (MIT) [25] include wavelengths and relative intensities of about 340 lines of $\mathrm{Ag}$ I and $\mathrm{Ag}$ II in the range $2000 \AA$ to $8274 \AA$. Most of the lines ascribed to Ag II in these tables have been observed by Kalus et al. [1] and Gilbert [2]. The MIT tables should be used with caution, as most Ag II wavelengths referred to MIT measurements, including those given with three places after the decimal point, appear to possess a large systematic shift on the order of $0.2 \AA$. Because of this shift, I did not make use of these tables.

\section{Optimized Energy Levels}

After the systematic shifts have been removed, and wavelength measurement uncertainties have been assessed, the level optimization is a straightforward procedure. I used the least-squares level optimization code LOPT [16] and included only the measurements of Kalus et al. [1], Rasmussen [3,4], Blair [7], and Shenstone [6], which makes a total of 450 lines. The level-optimization problem defined by this line list has 353 degrees of freedom (DF). The resulting ratio of the residual sum of squares (RSS) to the number of degrees of freedom RSS/DF $=0.78$, which indicates that the wavelength values and their uncertainties are statistically reasonable. The optimized energy levels and their uncertainties are given in Table 2. 
Table 2. Optimized energy levels of Ag II

\begin{tabular}{|c|c|c|c|c|c|c|c|c|}
\hline Configuration & Term & 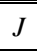 & Level $^{\mathrm{a}}\left(\mathrm{cm}^{-1}\right)$ & Landé $g$ & \multicolumn{3}{|c|}{ Leading percentages } & Alternate designation \\
\hline $4 d^{10}$ & ${ }^{1} \mathrm{~S}$ & 0 & $0.00(6)$ & & 99 & & & \\
\hline $4 d^{9}\left({ }^{2} D_{5 / 2}\right) 5 s$ & ${ }^{2}[5 / 2]$ & 3 & 39168.0177(16) & 1.318 & 100 & & & or $99^{3} \mathrm{D}$ \\
\hline $4 d^{9}\left({ }^{2} D_{5 / 2}\right) 5 s$ & ${ }^{2}[5 / 2]$ & 2 & 40745.3671(13) & 1.119 & 85 & $+154 d^{9}\left({ }^{2} D_{3 / 2}\right) 5 s$ & ${ }^{2}[3 / 2]$ & or $77^{3} \mathrm{D}+22^{1} \mathrm{D}$ \\
\hline $4 d^{9}\left(D_{3 / 2}^{2}\right) 5 s$ & ${ }^{2}[3 / 2]$ & 1 & 43742.7348 & 0.488 & 100 & & & or $99^{3} \mathrm{D}$ \\
\hline $4 d^{9}\left({ }^{2} D_{3 / 2}\right) 5 s$ & ${ }^{2}[3 / 2]$ & 2 & 46049.0620(13) & 1.028 & 85 & $+144 d^{9}\left({ }^{2} D_{5 / 2}\right) 5 s$ & ${ }^{2}[5 / 2]$ & or $77^{1} \mathrm{D}+22^{3} \mathrm{D}$ \\
\hline $4 d^{9}\left({ }^{2} D_{5 / 2}\right) 5 p$ & ${ }^{2}[3 / 2]^{\circ}$ & 2 & $80176.4601(12)$ & 1.464 & 92 & $+\quad 54 d^{9}\left({ }^{2} D_{5 / 2}\right) 5 p$ & ${ }^{2}[5 / 2]^{\circ}$ & or $91{ }^{3} \mathrm{P}^{\circ}+6{ }^{3} \mathrm{D}^{\circ}$ \\
\hline $4 d^{9}\left({ }^{2} D_{5 / 2}\right) 5 p$ & ${ }^{2}[7 / 2]^{\circ}$ & 3 & $82171.7320(13)$ & 1.059 & 89 & $+94 d^{9}\left({ }^{2} D_{5 / 2}\right) 5 p$ & ${ }^{2}[5 / 2]^{\circ}$ & or $56{ }^{3} \mathrm{~F}^{\circ}+36{ }^{1} \mathrm{~F}^{\circ}$ \\
\hline $4 d^{9}\left({ }^{2} D_{5 / 2}\right) 5 p$ & ${ }^{2}[3 / 2]^{\circ}$ & 1 & 83625.5147 & 1.410 & 57 & $+424 d^{9}\left({ }^{2} D_{3 / 2}\right) 5 p$ & ${ }^{2}[1 / 2]^{\circ}$ & or $88{ }^{3} \mathrm{P}^{\circ}+5^{3} \mathrm{D}^{\circ}$ \\
\hline $4 d^{9}\left({ }^{2} D_{5 / 2}\right) 5 p$ & ${ }^{2}[7 / 2]^{\circ}$ & 4 & 83669.6491(19) & 1.249 & 99 & & & or $99^{3} \mathrm{~F}^{\circ}$ \\
\hline $4 d^{9}\left({ }^{2} D_{5 / 2}\right) 5 p$ & ${ }^{2}[5 / 2]^{\circ}$ & 2 & $85200.7563(12)$ & 0.851 & 63 & $+314 d^{9}\left({ }^{2} D_{3 / 2}\right) 5 p$ & ${ }^{2}[5 / 2]^{\circ}$ & or $49{ }^{3} \mathrm{~F}^{\circ}+23{ }^{3} \mathrm{D}^{\circ}$ \\
\hline $4 d^{9}\left({ }^{2} D_{3 / 2}\right) 5 p$ & ${ }^{2}[1 / 2]^{\circ}$ & 0 & $86140.0853(17)$ & & 99 & & & or $98{ }^{3} \mathrm{P}^{\circ}$ \\
\hline $4 d^{9}\left({ }^{2} D_{5 / 2}\right) 5 p$ & ${ }^{2}[5 / 2]^{\circ}$ & 3 & $86460.6511(13)$ & 1.240 & 86 & $+104 d^{9}\left({ }^{2} D_{5 / 2}\right) 5 p$ & ${ }^{2}[7 / 2]^{\circ}$ & or $73{ }^{3} \mathrm{D}^{\circ}+24{ }^{1} \mathrm{~F}^{\circ}$ \\
\hline $4 d^{9}\left({ }^{2} D_{3 / 2}\right) 5 p$ & ${ }^{2}[5 / 2]^{\circ}$ & 2 & 86888.0990(12) & 0.889 & 64 & $+\quad 314 d^{9}\left({ }^{2} D_{5 / 2}\right) 5 p$ & ${ }^{2}[5 / 2]^{\circ}$ & or $46{ }^{1} \mathrm{D}^{\circ}+45^{3} \mathrm{~F}^{\circ}$ \\
\hline $4 d^{9}\left({ }^{2} D_{3 / 2}\right) 5 p$ & ${ }^{2}[5 / 2]^{\circ}$ & 3 & $89134.7240(13)$ & 1.076 & 96 & & & or $42{ }^{3} \mathrm{~F}^{\circ}+38{ }^{1} \mathrm{~F}^{\circ}$ \\
\hline $4 d^{9}\left({ }^{2} D_{3 / 2}\right) 5 p$ & ${ }^{2}[3 / 2]^{\circ}$ & 1 & $89895.5377(12)$ & 1.008 & 68 & $+184 d^{9}\left({ }^{2} D_{3 / 2}\right) 5 p$ & ${ }^{2}[1 / 2]^{\circ}$ & or $52{ }^{1} \mathrm{P}^{\circ}+46{ }^{3} \mathrm{D}^{\circ}$ \\
\hline $4 d^{9}\left({ }^{2} D_{3 / 2}\right) 5 p$ & ${ }^{2}[1 / 2]^{\circ}$ & 1 & $90334.8716(13)$ & 0.52 & 39 & $+\quad 304 d^{9}\left({ }^{2} D_{3 / 2}\right) 5 p$ & ${ }^{2}[3 / 2]^{\circ}$ & or $47^{3} \mathrm{D}^{\circ}+42{ }^{1} \mathrm{P}^{\circ}$ \\
\hline $4 d^{9}\left({ }^{2} D_{3 / 2}\right) 5 p$ & ${ }^{2}[3 / 2]^{\circ}$ & 2 & $90887.8423(12)$ & 1.099 & 92 & $+\quad 54 \mathrm{~d}^{9}\left({ }^{2} \mathrm{D}_{3 / 2}\right) 5 \mathrm{p}$ & ${ }^{2}[5 / 2]^{\circ}$ & or $61{ }^{3} \mathrm{D}^{\circ}+30{ }^{1} \mathrm{D}^{\circ}$ \\
\hline $4 d^{8} 5 s^{2}$ & ${ }^{3} \mathrm{~F}$ & 4 & 93932.926(3) & & 96 & & & \\
\hline $4 d^{8} 5 s^{2}$ & ${ }^{3} \mathrm{~F}$ & 3 & $97962.039(6)$ & & 97 & & & \\
\hline $4 d^{8} 5 s^{2}$ & ${ }^{3} \mathrm{~F}$ & 2 & 99606.031(6) & & 81 & $+144 d^{8} 5 s^{2}$ & ${ }^{1} \mathrm{D}$ & \\
\hline $4 d^{8} 5 s^{2}$ & ${ }^{3} \mathrm{P}$ & 2 & 105262.2691(16) & & 62 & $+244 d^{8} 5 s^{2}$ & ${ }^{1} \mathrm{D}$ & \\
\hline $4 d^{8} 5 s^{2}$ & ${ }^{3} \mathrm{P}$ & 1 & $108938.4282(17)$ & & 96 & & & \\
\hline $4 d^{8} 5 s^{2}$ & ${ }^{3} \mathrm{P}$ & 0 & 109127.691(3) & & 94 & & & \\
\hline $4 d^{8} 5 s^{2}$ & ${ }^{1} \mathrm{D}$ & 2 & 110773.5186(13) & & 59 & $+324 d^{8} 5 s^{2}$ & ${ }^{3} \mathrm{P}$ & \\
\hline $4 d^{8} 5 s^{2}$ & ${ }^{1} \mathrm{G}$ & 4 & $113602.1364(20)$ & & 95 & & & \\
\hline $4 d^{9}\left(D_{5 / 2}^{2}\right) 6 s$ & ${ }^{2}[5 / 2]$ & 3 & 120533.6058(16) & 1.293 & 100 & & & or $100{ }^{3} \mathrm{D}$ \\
\hline $4 d^{9}\left(D_{5 / 2}^{2}\right) 6 s$ & ${ }^{2}[5 / 2]$ & 2 & 120911.2048(14) & 1.195 & 99 & & & or $52{ }^{1} \mathrm{D}+47{ }^{3} \mathrm{D}$ \\
\hline $4 d^{9}\left(D_{3 / 2}^{2}\right) 6 s$ & ${ }^{2}[3 / 2]$ & 1 & $125126.4097(14)$ & 0.53 & 100 & & & or $100^{3} \mathrm{D}$ \\
\hline $4 d^{9}\left({ }^{2} D_{3 / 2}\right) 6 s$ & ${ }^{2}[3 / 2]$ & 2 & $125404.8818(14)$ & 1.11 & 99 & & & or $52{ }^{3} \mathrm{D}+47^{1} \mathrm{D}$ \\
\hline $4 d^{9}\left({ }^{2} D_{5 / 2}\right) 5 d$ & ${ }^{2}[1 / 2]$ & 1 & $125574.8546(24)$ & 1.84 & 98 & & & or $73{ }^{3} \mathrm{~S}+20{ }^{3} \mathrm{P}$ \\
\hline $4 d^{9}\left({ }^{2} D_{5 / 2}\right) 5 d$ & ${ }^{2}[9 / 2]$ & 5 & 126668.189(3) & & 100 & & & or $99{ }^{3} \mathrm{G}$ \\
\hline $4 d^{9}\left({ }^{2} D_{5 / 2}\right) 5 d$ & ${ }^{2}[9 / 2]$ & 4 & $126679.4151(22)$ & 1.03 & 99 & & & or $50{ }^{3} \mathrm{G}+48{ }^{1} \mathrm{G}$ \\
\hline $4 d^{9}\left({ }^{2} D_{5 / 2}\right) 5 d$ & ${ }^{2}[3 / 2]$ & 2 & 126766.4681(19) & 1.334 & 94 & & & or $68{ }^{3} \mathrm{P}+29^{3} \mathrm{D}$ \\
\hline $4 d^{9}\left({ }^{2} D_{5 / 2}\right) 5 d$ & ${ }^{2}[3 / 2]$ & 1 & $126770.2987(16)$ & 0.90 & 99 & & & or $50{ }^{1} \mathrm{P}+26{ }^{3} \mathrm{D}$ \\
\hline $4 d^{9}\left({ }^{2} D_{5 / 2}\right) 5 d$ & ${ }^{2}[5 / 2]$ & 3 & 127211.1186(16) & 1.288 & 96 & & & or $80{ }^{3} \mathrm{D}+16{ }^{3} \mathrm{~F}$ \\
\hline $4 d^{9}\left({ }^{2} D_{5 / 2}\right) 5 d$ & ${ }^{2}[7 / 2]$ & 3 & $127491.0946(18)$ & 0.958 & 96 & & & or $52{ }^{1} \mathrm{~F}+29{ }^{3} \mathrm{~F}$ \\
\hline $4 d^{9}\left({ }^{2} D_{5 / 2}\right) 5 d$ & ${ }^{2}[5 / 2]$ & 2 & 127523.4831(15) & 1.006 & 95 & & & or $51{ }^{1} \mathrm{D}+23^{3} \mathrm{D}$ \\
\hline $4 d^{9}\left({ }^{2} D_{5 / 2}\right) 5 d$ & ${ }^{2}[7 / 2]$ & 4 & $127608.6762(23)$ & 1.218 & 99 & & & or $84{ }^{3} F+11{ }^{1} \mathrm{G}$ \\
\hline $4 d^{8}\left({ }^{3} F\right) 5 s 5 p\left({ }^{3} P^{\circ}\right)$ & ${ }^{5} \mathrm{D}^{\circ}$ & 4 & $128014.134(5)$ & & & $+64 d^{8}\left({ }^{3} F\right) 5 s 5 p\left({ }^{3} \mathrm{P}^{\circ}\right)$ & ${ }^{5} \mathrm{~F}^{\circ}$ & \\
\hline $4 d^{9}\left({ }^{2} D_{5 / 2}\right) 5 d$ & ${ }^{2}[1 / 2]$ & 0 & $128535.1327(24)$ & & 77 & $+224 d^{9}\left(D_{3 / 2}\right) 5 d$ & ${ }^{2}[1 / 2]$ & or $85{ }^{3} \mathrm{P}+14{ }^{1} \mathrm{~S}$ \\
\hline $4 d^{8}\left({ }^{3} \mathrm{~F}\right) 5 \mathrm{~s} 5 \mathrm{p}\left({ }^{3} \mathrm{P}^{\circ}\right)$ & ${ }^{5} \mathrm{D}^{\circ}$ & 3 & 130725.061(22) & & 77 & $+84 d^{8}\left({ }^{3} F\right) 5 s 5 p\left({ }^{3} P^{\circ}\right)$ & ${ }^{5} \mathrm{~F}^{\circ}$ & \\
\hline $4 d^{9}\left({ }^{2} D_{3 / 2}\right) 5 d$ & ${ }^{2}[1 / 2]$ & 1 & 130762.6969(18) & 1.51 & 98 & & & or $47{ }^{3} \mathrm{P}+27{ }^{1} \mathrm{P}$ \\
\hline
\end{tabular}




\begin{tabular}{|c|c|c|c|c|c|c|c|}
\hline Configuration & Term & $J$ & $\operatorname{Level}^{\mathrm{a}}\left(\mathrm{cm}^{-1}\right)$ & Landé $g$ & Leading percentages & & Alternate designation \\
\hline $4 d^{9}\left(D_{3 / 2}^{2}\right) 5 d$ & ${ }^{2}[7 / 2]$ & 3 & $131252.5017(22)$ & $0.75 ?$ & 99 & & or $84^{3} \mathrm{G}+10{ }^{1} \mathrm{~F}$ \\
\hline $4 d^{8}\left({ }^{3} F\right) 5 s 5 p\left({ }^{3} P^{\circ}\right)$ & ${ }^{5} G^{\circ}$ & 5 & 131470.919(4) & & $60+314 d^{8}\left({ }^{3} \mathrm{~F}\right) 5 \mathrm{~s} 5 \mathrm{p}\left({ }^{3} \mathrm{P}^{\circ}\right)$ & ${ }^{5} \mathrm{~F}^{\circ}$ & \\
\hline $4 d^{9}\left({ }^{2} D_{3 / 2}\right) 5 d$ & ${ }^{2}[3 / 2]$ & 1 & 131507.1120(18) & 0.74 & 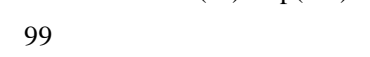 & & or $72{ }^{3} \mathrm{D}+17{ }^{1} \mathrm{P}$ \\
\hline $4 d^{9}\left({ }^{2} D_{3 / 2}\right) 5 d$ & ${ }^{2}[7 / 2]$ & 4 & 131517.079(3) & 1.065 & 99 & & or $45^{3} \mathrm{G}+40{ }^{1} \mathrm{G}$ \\
\hline $4 d^{9}\left({ }^{2} D_{5 / 2}\right) 6 p$ & ${ }^{2}[3 / 2]^{\circ}$ & 2 & $131577.02(7)$ & & $92+74 d^{9}\left({ }^{2} D_{5 / 2}\right) 6 p$ & ${ }^{2}[5 / 2]^{\circ}$ & or $86^{3} \mathrm{P}^{\circ}+13^{3} \mathrm{D}^{\circ}$ \\
\hline $4 d^{9}\left(D_{3 / 2}^{2}\right) 5 d$ & ${ }^{2}[3 / 2]$ & 2 & 131790.3202(16) & 1.147 & 98 & & or $46^{3} \mathrm{D}+26^{1} \mathrm{D}$ \\
\hline $4 d^{9}\left({ }^{2} D_{5 / 2}\right) 6 p$ & ${ }^{2}[7 / 2]^{\circ}$ & 3 & $132017.588(14)$ & & $87+124 d^{9}\left({ }^{2} D_{5 / 2}\right) 6 p$ & ${ }^{2}[5 / 2]^{\circ}$ & or $49^{3} \mathrm{~F}^{\circ}+41^{1} \mathrm{~F}^{\circ}$ \\
\hline $4 d^{9}\left({ }^{2} D_{3 / 2}\right) 5 d$ & ${ }^{2}[5 / 2]$ & 2 & $132155.6138(15)$ & 0.71 & 98 & & or $766^{3} \mathrm{~F}+20^{1} \mathrm{D}$ \\
\hline $4 d^{9}\left(D_{3 / 2}^{2}\right) 5 d$ & ${ }^{2}[5 / 2]$ & 3 & $132198.3472(21)$ & 1.095 & 99 & & or $48{ }^{3} \mathrm{~F}+35{ }^{1} \mathrm{~F}$ \\
\hline $4 d^{9}\left({ }^{2} D_{5 / 2}\right) 6 p$ & ${ }^{2}[7 / 2]^{\circ}$ & 4 & $132429.668(3)$ & & 96 & & or $96{ }^{3} \mathrm{~F}^{\circ}$ \\
\hline $4 d^{8}\left({ }^{3} \mathrm{~F}\right) 5 \mathrm{~s} 5 \mathrm{p}\left({ }^{3} \mathrm{P}^{\circ}\right)$ & ${ }^{5} G^{\circ}$ & 4 & $132688.679(7)$ & & $61+154 d^{8}\left({ }^{3} \mathrm{~F}\right) 5 \mathrm{~s} 5 \mathrm{p}\left({ }^{3} \mathrm{P}^{\circ}\right)$ & ${ }^{5} \mathrm{~F}^{\circ}$ & \\
\hline $4 d^{9}\left({ }^{2} D_{5 / 2}\right) 6 p$ & ${ }^{2}[3 / 2]^{\circ}$ & 1 & 132841.701(5) & & 96 & & or $46^{3} \mathrm{P}^{\circ}+44^{1} \mathrm{P}^{\circ}$ \\
\hline $4 d^{9}\left(D_{5 / 2}^{2}\right) 6 p$ & ${ }^{2}[5 / 2]^{\circ}$ & 2 & $132920.339(4)$ & & $87+64 d^{9}\left({ }^{2} D_{5 / 2}\right) 6 p$ & ${ }^{2}[3 / 2]^{\circ}$ & or $53{ }^{1} \mathrm{D}^{\circ}+28{ }^{3} \mathrm{D}^{\circ}$ \\
\hline $4 d^{9}\left({ }^{2} D_{5 / 2}\right) 6 p$ & ${ }^{2}[5 / 2]^{\circ}$ & 3 & 133022.073(3) & & $87+104 d^{9}\left({ }^{2} D_{5 / 2}\right) 6 p$ & ${ }^{2}[7 / 2]^{\circ}$ & or $81^{3} \mathrm{D}^{\circ}+18{ }^{1} \mathrm{~F}^{\circ}$ \\
\hline $4 d^{8}\left({ }^{3} F\right) 5 s 5 p\left({ }^{3} P^{\circ}\right)$ & ${ }^{5} \mathrm{D}^{\circ}$ & 2 & 133141.85(3) & & $76+114 d^{8}\left({ }^{3} \mathrm{P}\right) 5 \mathrm{~s} 5 \mathrm{p}\left({ }^{3} \mathrm{P}^{\circ}\right)$ & ${ }^{5} \mathrm{D}^{\circ}$ & \\
\hline $4 d^{8}\left({ }^{3} \mathrm{~F}\right) 5 \mathrm{~s} 5 \mathrm{p}\left({ }^{3} \mathrm{P}^{\circ}\right)$ & ${ }^{5} G^{\circ}$ & 3 & $134343.135(7)$ & & $74+144 d^{8}\left({ }^{3} \mathrm{~F}\right) 5 \mathrm{~s} 5 \mathrm{p}\left({ }^{3} \mathrm{P}^{\circ}\right)$ & ${ }^{5} \mathrm{~F}^{\circ}$ & \\
\hline $4 d^{9}\left({ }^{2} D_{3 / 2}\right) 5 d$ & ${ }^{2}[1 / 2]$ & 0 & $134455.9504(21)$ & & $76+224 d^{9}\left({ }^{2} D_{5 / 2}\right) 5 d$ & ${ }^{2}[1 / 2]$ & or $78{ }^{1} \mathrm{~S}+14{ }^{3} \mathrm{P}$ \\
\hline $4 d^{8}\left({ }^{3} F\right) 5 s 5 p\left({ }^{3} P^{\circ}\right)$ & ${ }^{5} \mathrm{D}^{\circ}$ & 1 & 134799.164(4) & & $80+144 d^{8}\left({ }^{3} \mathrm{P}\right) 5 \mathrm{~s} 5 \mathrm{p}\left({ }^{3} \mathrm{P}^{\circ}\right)$ & ${ }^{5} \mathrm{D}^{\circ}$ & \\
\hline $4 d^{8}\left({ }^{3} \mathrm{~F}\right) 5 \mathrm{~s} 5 \mathrm{p}\left({ }^{3} \mathrm{P}^{\circ}\right)$ & ${ }^{5} G^{\circ}$ & 2 & 135415.323(9) & & $82+84 d^{8}\left({ }^{3} \mathrm{~F}\right) 5 \mathrm{~s} 5 \mathrm{p}\left({ }^{3} \mathrm{P}^{\circ}\right)$ & ${ }^{5} \mathrm{~F}^{\circ}$ & \\
\hline $4 d^{8}\left({ }^{3} F\right) 5 s 5 p\left({ }^{3} P^{\circ}\right)$ & ${ }^{5} \mathrm{~F}^{\circ}$ & 4 & $136107.052(9)$ & & $63+114 d^{8}\left({ }^{3} \mathrm{~F}\right) 5 \mathrm{~s} 5 \mathrm{p}\left({ }^{3} \mathrm{P}^{\circ}\right)$ & ${ }^{3} \mathrm{G}^{\circ}$ & \\
\hline $4 d^{9}\left({ }^{2} D_{3 / 2}\right) 6 p$ & ${ }^{2}[1 / 2]^{\circ}$ & 0 & $136169.811(22) ?$ & & 99 & & or $99^{3} \mathrm{P}^{\circ}$ \\
\hline $4 d^{9}\left({ }^{2} D_{3 / 2}\right) 6 p$ & ${ }^{2}[5 / 2]^{\circ}$ & 2 & $136518.895(17)$ & & $94+54 \mathrm{~d}^{9}\left({ }^{2} \mathrm{D}_{3 / 2}\right) 6 \mathrm{p}$ & ${ }^{2}[3 / 2]^{\circ}$ & or $85^{3} \mathrm{~F}^{\circ}+14^{1} \mathrm{D}^{\circ}$ \\
\hline $4 d^{9}\left(D^{2} D_{3 / 2}\right) 6 p$ & ${ }^{2}[1 / 2]^{\circ}$ & 1 & $136832.527(14)$ & & 93 & & or $544^{1} \mathrm{p}^{\circ}+42^{3} \mathrm{P}^{\circ}$ \\
\hline $4 d^{9}\left({ }^{2} D_{3 / 2}\right) 6 p$ & ${ }^{2}[5 / 2]^{\circ}$ & 3 & 137176.52(3) & & 89 & & or $45^{3} \mathrm{~F}^{\circ}+38{ }^{1} \mathrm{~F}^{\circ}$ \\
\hline $4 d^{9}\left({ }^{2} D_{3 / 2}\right) 6 p$ & ${ }^{2}[3 / 2]^{\circ}$ & 1 & $137225.409(7)$ & & $84+54 d^{8} 5 s 5 p$ & & or $77^{3} \mathrm{D}^{\circ}+11^{3} \mathrm{P}^{\circ}$ \\
\hline $4 d^{8}\left({ }^{3} \mathrm{~F}\right) 5 \mathrm{~s} 5 \mathrm{p}\left({ }^{3} \mathrm{P}^{\circ}\right)$ & ${ }^{5} \mathrm{~F}^{\circ}$ & 3 & $137435.220(12)$ & & $53+154 d^{8}\left({ }^{3} \mathrm{~F}\right) 5 \mathrm{~s} 5 \mathrm{p}\left({ }^{3} \mathrm{P}^{\circ}\right)$ & ${ }^{5} \mathrm{G}^{\circ}$ & \\
\hline $4 d^{9}\left({ }^{2} D_{3 / 2}\right) 6 p$ & ${ }^{2}[3 / 2]^{\circ}$ & 2 & $137468.255(10)$ & & 79 & & or $48^{3} \mathrm{D}^{\circ}+27^{1} \mathrm{D}^{\circ}$ \\
\hline $4 d^{8}\left({ }^{3} \mathrm{~F}\right) 5 \mathrm{~s} 5 \mathrm{p}\left({ }^{3} \mathrm{P}^{\circ}\right)$ & ${ }^{3} \mathrm{D}^{\circ}$ & 3 & 138635.91(6) & & $48+174 d^{8}\left({ }^{3} \mathrm{~F}\right) 5 \mathrm{~s} 5 \mathrm{p}\left({ }^{3} \mathrm{P}^{\circ}\right)$ & ${ }^{3} \mathrm{~F}^{\circ}$ & \\
\hline $4 d^{8}\left({ }^{3} F\right) 5 s 5 p\left({ }^{3} P^{\circ}\right)$ & ${ }^{3} \mathrm{D}^{\circ}$ & 2 & 139230.01(6) & & $25+174 d^{8}\left({ }^{3} \mathrm{~F}\right) 5 \mathrm{~s} 5 \mathrm{p}\left({ }^{3} \mathrm{P}^{\circ}\right)$ & ${ }^{5} \mathrm{~F}^{\circ}$ & \\
\hline $4 d^{8}\left({ }^{3} F\right) 5 s 5 p\left({ }^{3} P^{\circ}\right)$ & ${ }^{3} \mathrm{G}^{\circ}$ & 3 & $140598.68(6)$ & & $51+224 d^{8}\left({ }^{3} \mathrm{~F}\right) 5 \mathrm{~s} 5 \mathrm{p}\left({ }^{3} \mathrm{P}^{\circ}\right)$ & ${ }^{3} \mathrm{D}^{\circ}$ & \\
\hline $4 d^{8}\left({ }^{3} \mathrm{P}\right) 5 \mathrm{~s} 5 \mathrm{p}\left({ }^{3} \mathrm{P}^{\circ}\right)$ & ${ }^{5} \mathrm{P}^{\circ}$ & 2 & $140964.44(6)$ & & $58+184 d^{8}\left({ }^{3} \mathrm{~F}\right) 5 \mathrm{~s} 5 \mathrm{p}\left({ }^{3} \mathrm{P}^{\circ}\right)$ & ${ }^{3} \mathrm{D}^{\circ}$ & \\
\hline $4 d^{8}\left({ }^{3} \mathrm{P}\right) 5 \mathrm{~s} 5 \mathrm{p}\left({ }^{3} \mathrm{P}^{\circ}\right)$ & ${ }^{5} \mathrm{P}^{\circ}$ & 1 & $140988.36(6)$ & & $50+164 d^{8}\left({ }^{3} \mathrm{~F}\right) 5 \mathrm{~s} 5 \mathrm{p}\left({ }^{3} \mathrm{P}^{\circ}\right)$ & ${ }^{5} \mathrm{~F}^{\circ}$ & \\
\hline $4 d^{8}\left({ }^{3} P\right) 5 s 5 p\left({ }^{3} P^{\circ}\right)$ & ${ }^{5} \mathrm{P}^{\circ}$ & 3 & $141152.11(6)$ & & $62+214 d^{8}\left({ }^{3} \mathrm{~F}\right) 5 \mathrm{~s} 5 \mathrm{p}\left({ }^{3} \mathrm{P}^{\circ}\right)$ & ${ }^{3} \mathrm{G}^{\circ}$ & \\
\hline $4 d^{8}\left({ }^{3} F\right) 5 s 5 p\left({ }^{3} P^{\circ}\right)$ & ${ }^{3} \mathrm{~F}^{\circ}$ & 3 & $141920.32(6)$ & & $33+294 \mathrm{~d}^{8}\left({ }^{3} \mathrm{~F}\right) 5 \mathrm{~s} 5 \mathrm{p}\left({ }^{3} \mathrm{P}^{\circ}\right)$ & ${ }^{1} \mathrm{~F}^{\circ}$ & \\
\hline $4 d^{8}\left({ }^{3} F\right) 5 s 5 p\left({ }^{3} P^{\circ}\right)$ & ${ }^{3} \mathrm{D}^{\circ}$ & 1 & 142346.06(6) & & $36+404 d^{8}\left({ }^{3} \mathrm{P}\right) 5 \mathrm{~s} 5 \mathrm{p}\left({ }^{3} \mathrm{P}^{\circ}\right)$ & ${ }^{5} \mathrm{P}^{\circ}$ & \\
\hline $4 d^{8}\left({ }^{3} F\right) 5 s 5 p\left({ }^{3} P^{\circ}\right)$ & ${ }^{3} \mathrm{~F}^{\circ}$ & 2 & $143743.90(6)$ & & $55+194 d^{8}\left({ }^{3} \mathrm{~F}\right) 5 \mathrm{~s} 5 \mathrm{p}\left({ }^{3} \mathrm{P}^{\circ}\right)$ & ${ }^{3} \mathrm{D}^{\circ}$ & \\
\hline $4 d^{9}\left({ }^{2} D_{5 / 2}\right) 7 s$ & ${ }^{2}[5 / 2]$ & 3 & $144478.44(9)$ & & 100 & & or $100^{3} \mathrm{D}$ \\
\hline $4 d^{9}\left({ }^{2} D_{5 / 2}\right) 7 s$ & ${ }^{2}[5 / 2]$ & 2 & $144624.16(7)$ & & 100 & & or $57^{1} \mathrm{D}+43^{3} \mathrm{D}$ \\
\hline $4 d^{9}\left({ }^{2} D_{5 / 2}\right) 4 f$ & ${ }^{2}[1 / 2]^{\circ}$ & 1 & $145014.040(4)$ & & $82+174 d^{9}\left({ }^{2} D_{5 / 2}\right) 4 f$ & ${ }^{2}[3 / 2]^{\circ}$ & or $80^{3} \mathrm{P}^{\circ}+13^{3} \mathrm{D}^{\circ}$ \\
\hline $4 d^{8}\left({ }^{3} \mathrm{~F}\right) 5 \mathrm{~s} 5 \mathrm{p}\left({ }^{3} \mathrm{P}^{\circ}\right)$ & ${ }^{1} \mathrm{D}^{\circ}$ & 2 & $145035.112(6)$ & & $34+134 d^{9} 4 f$ & ${ }^{3} \mathrm{P}^{\circ}$ & \\
\hline $4 d^{9}\left(D^{2} D_{5 / 2}\right) 4 f$ & ${ }^{2}[11 / 2]^{\circ}$ & 6 & $145181.205(4)$ & & 100 & & or $99{ }^{3} \mathrm{H}^{\circ}$ \\
\hline $\begin{array}{l}4 d^{9}\left({ }^{2} D_{5 / 2}\right) 4 f \\
4 d^{9}\left(^{2} D_{5 / 2}\right) 4 f\end{array}$ & $\begin{array}{l}{ }^{2}[11 / 2]^{\circ} \\
{ }^{2}[3 / 2]^{\circ}\end{array}$ & $\begin{array}{l}5 \\
2\end{array}$ & $\begin{array}{l}145182.054(4) \\
145197.750(4)\end{array}$ & & $\left.144 d^{9} c^{2} D-\gamma\right) 4 f$ & ${ }^{2}[5 / 2]^{\circ}$ & or $55{ }^{1} \mathrm{H}^{\circ}+45^{3} \mathrm{H}^{\circ}$ \\
\hline
\end{tabular}




\begin{tabular}{|c|c|c|c|c|c|c|c|}
\hline Configuration & Term & $\bar{J}$ & $\operatorname{Level}^{\mathrm{a}}\left(\mathrm{cm}^{-1}\right)$ & Landé $g$ & \multicolumn{2}{|l|}{ Leading percentages } & Alternate designation \\
\hline $4 d^{9}\left({ }^{2} D_{5 / 2}\right) 4 f$ & ${ }^{2}[3 / 2]^{\circ}$ & 1 & $145242.847(5)$ & & $83+164 d^{9}\left({ }^{2} D_{5 / 2}\right) 4 f$ & ${ }^{2}[1 / 2]^{\circ}$ & or $51^{1} \mathrm{P}^{\circ}+47^{3} \mathrm{D}^{\circ}$ \\
\hline $4 d^{9}\left({ }^{2} D_{5 / 2}\right) 4 f$ & ${ }^{2}[5 / 2]^{\circ}$ & 2 & 145309.528(6) & & $74+94 d^{9}\left({ }^{2} D_{5 / 2}\right) 4 f$ & ${ }^{2}[3 / 2]^{\circ}$ & or $42{ }^{1} \mathrm{D}^{\circ}+32^{3} \mathrm{~F}^{\circ}$ \\
\hline $4 d^{9}\left({ }^{2} D_{5 / 2}\right) 4 f$ & ${ }^{2}[9 / 2]^{\circ}$ & 5 & 145389.480(3) & & 99 & & or $86^{3} \mathrm{G}^{\circ}+8{ }^{3} \mathrm{H}^{\circ}$ \\
\hline $4 d^{9}\left({ }^{2} D_{5 / 2}\right) 4 f$ & ${ }^{2}[7 / 2]^{\circ}$ & 3 & $145408.300(3)$ & & 93 & & or $40^{3} \mathrm{~F}^{\circ}+29^{1} \mathrm{~F}^{\circ}$ \\
\hline $4 d^{9}\left(D^{2} D_{5 / 2}\right) 4 f$ & ${ }^{2}[7 / 2]^{\circ}$ & 4 & 145427.113(3) & & $50+494 \mathrm{~d}^{9}\left({ }^{2} \mathrm{D}_{5 / 2}\right) 4 \mathrm{f}$ & ${ }^{2}[9 / 2]^{\circ}$ & or $47^{3} \mathrm{G}^{\circ}+39^{3} \mathrm{~F}^{\circ}$ \\
\hline $4 d^{9}\left(D_{5 / 2}^{2}\right) 4 f$ & ${ }^{2}[9 / 2]^{\circ}$ & 4 & 145497.860(3) & & $49+484 d^{9}\left({ }^{2} D_{5 / 2}\right) 4 f$ & ${ }^{2}[7 / 2]^{\circ}$ & or $51{ }^{1} \mathrm{G}^{\circ}+34{ }^{3} \mathrm{~F}^{\circ}$ \\
\hline $4 d^{8}\left({ }^{3} F\right) 5 s 5 p\left({ }^{3} P^{\circ}\right)$ & ${ }^{1} \mathrm{~F}^{\circ}$ & 3 & $145558.556(6)$ & & $27+134 \mathrm{~d}^{9} 4 \mathrm{f}$ & ${ }^{3} \mathrm{D}^{\circ}$ & \\
\hline $4 d^{8}\left({ }^{1} \mathrm{D}\right) 5 \mathrm{~s} 5 \mathrm{p}\left({ }^{3} \mathrm{P}^{\circ}\right)$ & ${ }^{3} \mathrm{~F}^{\circ}$ & 2 & $146123.25(6)$ & & $46+124 d^{8}\left({ }^{3} \mathrm{P}\right) 5 \mathrm{~s} 5 \mathrm{p}\left({ }^{3} \mathrm{P}^{\circ}\right)$ & ${ }^{5} \mathrm{D}^{\circ}$ & \\
\hline $4 d^{9}\left({ }^{2} D_{3 / 2}\right) 7 s$ & ${ }^{2}[3 / 2]$ & 1 & $149082.86(9)$ & & & & or $99^{3} \mathrm{D}$ \\
\hline $4 d^{9}\left({ }^{2} D_{3 / 2}\right) 7 \mathrm{~s}$ & ${ }^{2}[3 / 2]$ & 2 & $149182.18(8)$ & & 100 & & or $57^{3} \mathrm{D}+42^{1} \mathrm{D}$ \\
\hline $4 d^{9}\left({ }^{2} D_{5 / 2}\right) 8 s$ & ${ }^{2}[5 / 2]$ & 3 & 155088.95(23) & & 100 & & or $100{ }^{3} \mathrm{D}$ \\
\hline $4 d^{9}\left(D^{2} D_{5 / 2}\right) 8 s$ & ${ }^{2}[5 / 2]$ & 2 & 155161.42(22) & & 100 & & or $59{ }^{1} \mathrm{D}+41^{3} \mathrm{D}$ \\
\hline $4 d^{9}\left({ }^{2} D_{3 / 2}\right) 8 s$ & ${ }^{2}[3 / 2]$ & 1 & $159695.21(24)$ & & 100 & & or $100{ }^{3} \mathrm{D}$ \\
\hline $4 d^{9}\left({ }^{2} D_{3 / 2}\right) 8 s$ & ${ }^{2}[3 / 2]$ & 2 & $159740.36(24)$ & & 100 & & or $58{ }^{3} \mathrm{D}+41^{1} \mathrm{D}$ \\
\hline Ag III $\left(4 d^{9}{ }^{2} D_{5 / 2}\right)$ & Limit & & $173283(7)$ & & & & \\
\hline Ag III $\left(4 d^{9}{ }^{2} D_{3 / 2}\right)$ & Limit & & $177892(7)$ & & & & \\
\hline
\end{tabular}

${ }^{a}$ Uncertainties of separations from the $4 \mathrm{~d}^{9}\left({ }^{2} \mathrm{D}_{5 / 2}\right) 5 \mathrm{p}{ }^{2}[3 / 2]_{1}$ level are given in parentheses after the value in units of the last decimal place of the value. To obtain the uncertainties of the excitation energies, they must be combined in quadrature with the uncertainty of the ground level, $\pm 0.06 \mathrm{~cm}^{-1}$. All uncertainties are given on the level of one standard deviation.

As noted by Kalus et al. [1], the uncertainty of the absolute level values is determined mainly by three observed spectral lines at 1106, 1112, and $1196 \AA$ connecting the $4 \mathrm{~d}^{10}{ }^{1} \mathrm{~S}_{0}$ ground level with the three $J=1$ levels of $4 \mathrm{~d}^{9} 5 \mathrm{p}\left({ }^{3} \mathrm{D}^{\circ}{ }_{1},{ }^{1} \mathrm{P}_{1}^{\circ}\right.$, and ${ }^{3} \mathrm{P}^{\circ}$, respectively). They estimated this uncertainty as $\pm 0.1 \mathrm{~cm}^{-1}$ and estimated the uncertainties of intervals between the excited levels to be in the range \pm 0.001 to $\pm 0.01 \mathrm{~cm}^{-1}$. The newly optimized energy levels given in Table 2 agree very well with those given by Kalus et al. [1]. Excitation energies of all levels are greater than those given by Kalus et al. by $0.04 \mathrm{~cm}^{-1}$ on average, which is well within the uncertainty $\pm 0.1 \mathrm{~cm}^{-1}$ specified by Kalus et al. Separations of all excited levels from $4 \mathrm{~d}^{9} 5 \mathrm{p}^{3} \mathrm{P}^{\circ}{ }_{1}$ are all within the range of uncertainties given by Kalus et al. The level $4 \mathrm{~d}^{9} 5 \mathrm{p}^{3} \mathrm{P}^{\circ}{ }_{1}$ was chosen as the base for determining the relative uncertainties of excited levels, because it has the greatest number of accurately measured connecting lines. In most cases, the number of significant figures given in the energy value is determined by the value of the relative uncertainty. In some cases, an extra digit was required in the energy value in order to exactly match some of the observed wavelengths.

The only questionable level in Table 2 is $4 \mathrm{~d}^{9} 6 \mathrm{p}^{3} \mathrm{P}^{\circ}{ }_{0}$, which is determined by a single line at $9052.70 \AA$ tentatively classified by Rasmussen [4] as a transition from this level to $4 \mathrm{~d}^{9} 6 \mathrm{~s}^{3} \mathrm{D}_{1}$. Another potentially strong transition from this level to $4 \mathrm{~d}^{9} 5 \mathrm{~s}^{3} \mathrm{D}_{1}$ is predicted to occur at 1081.9340 (3) $\AA$ and could have been masked by the much stronger line at $1082.1458 \AA$ in the spectrum observed by Kalus et al. [1].

The four $4 \mathrm{~d}^{9} 8 \mathrm{~s}$ levels are determined with uncertainties of about $0.2 \mathrm{~cm}^{-1}$ from lines of transitions to $4 d^{9} 5 p$ and $4 d^{9} 6 p$ measured by Gilbert [2].

The Landé factors included in Table 2 are from Moore [26]. Designations of the levels are discussed in Sec. 5. 


\section{Ionization Limit}

The previously accepted value of the first ionization limit, $173277.4 \mathrm{~cm}^{-1}$, was derived by Benschop et al. [5] from the $4 \mathrm{~d}^{9} n \mathrm{~s}(n=5-8)^{1,3} \mathrm{D}$ series. They noted that their newly identified (now discarded) $4 \mathrm{~d}^{9} 9 \mathrm{~s}{ }^{1,3} \mathrm{D}$ levels are "slightly perturbed" and did not include them in the derivation of the limit. Benschop et al. did not specify the uncertainty of their value. Since now the $4 \mathrm{~d}^{9} n$ s series are known much more accurately, it is possible to derive an improved limit value from the same series using similar quantumdefect expansion formulas. For that I used the computer program RITZPL by Sansonetti [27]. Each of the four four-member series was exactly fitted using the three-constant extended Ritz formula

$$
\delta_{n}=c_{0}+c_{1} /\left(n-\delta_{n}\right)^{2}+c_{2} /\left(n-\delta_{n}\right)^{4}+c_{3} /\left(n-\delta_{n}\right)^{6},
$$

where $c_{i}$ are the fitted constants and $\delta_{n}$ is the quantum defect describing an empirical correction to the principal quantum number $n$ required for the excitation energy $E_{n}$ to satisfy the hydrogenic formula:

$$
E_{\mathrm{I}}-E_{n}=R Z^{2} /\left(n-\delta_{n}\right)^{2},
$$

where $E_{\mathrm{I}}$ is the ionization energy, $Z$ is the charge of the ionic core, and $R$ is the mass-corrected Rydberg constant.

The mean of the four resulting values of the ionization limit is $173283 \mathrm{~cm}^{-1}$ with a standard deviation of $7 \mathrm{~cm}^{-1}$. In this derivation, I used the known value of the $4 \mathrm{~d}^{9}{ }^{2} \mathrm{D}_{5 / 2}{ }^{2} \mathrm{D}_{3 / 2}$ separation in $\mathrm{Ag}$ III, $4609.2 \mathrm{~cm}^{-1}$ [20].

To verify that higher-order terms omitted in Eq. (1) do not significantly influence the results, I made a similar derivation of the ionization limit for neutral palladium, where an accurate value of the limit, $67241.3(8) \mathrm{cm}^{-1}$ was derived by Baig et al. [28] from high-member absorption series $4 \mathrm{~d}^{10}{ }^{1} \mathrm{~S}_{0}-4 \mathrm{~d}^{9} n \mathrm{p}$ $(n=10-30)$ and $n f(n=9-17)$. For comparison with Ag II, only the $4 \mathrm{~d}^{9}{ }_{5 / 2} n \mathrm{~s}^{2}[5 / 2]_{3}$ series can be used in Pd I, because the $n=8$ members are not known in the Pd I $4 \mathrm{~d}_{3 / 2}^{9} n \mathrm{~s}^{2}[3 / 2]_{1}$ and ${ }^{2}[3 / 2]_{2}$ series, and the $4 \mathrm{~d}^{9}{ }_{5 / 2} n \mathrm{~s}^{2}[5 / 2]_{2}$ series is strongly perturbed. From the exact fit of the formulas (1) and (2) with the first four members $(n=5-8)$ of the $4 \mathrm{~d}_{5 / 2}^{9} n \mathrm{~s}^{2}[5 / 2]_{3}$ series precisely measured by Engleman et al. [8], I obtained the limit at $67241.0 \mathrm{~cm}^{-1}$, differing from the adopted value of Baig et al. by only $0.3 \mathrm{~cm}^{-1}$. This, as well as the small variation between the limit values obtained from the four different series, confirms the validity of the procedure for Ag II and allows me to adopt the resulting value of $173283(7) \mathrm{cm}^{-1}$ for the Ag II ionization limit.

\section{Theoretical Interpretation of the Energy Levels}

Atomic structure calculations were made in this work with the Cowan code package [29]. The following configuration sets were used: for even parity, $\left[4 \mathrm{p}^{6}\right] 4 \mathrm{~d}^{10}, 4 \mathrm{~d}^{9} n s, 4 \mathrm{~d}^{9} n \mathrm{~d}(n=5-9), 4 \mathrm{~d}^{9} 5 \mathrm{~g}, 4 \mathrm{~d}^{8} 5 \mathrm{~s}^{2}$, $4 \mathrm{~d}^{8} 5 \mathrm{p}^{2}, 4 \mathrm{~d}^{8} 5 \mathrm{~d}^{2}, 4 \mathrm{~d}^{8} 5 \mathrm{~s} 5 \mathrm{~d}, 4 \mathrm{~d}^{8} 5 \mathrm{pnf}(n=4,5), 4 \mathrm{p}^{5} 4 \mathrm{~d}^{10} 5 \mathrm{p}, 4 \mathrm{p}^{5} 4 \mathrm{~d}^{10} n \mathrm{f}(n=4,5)$; for odd parity, [4 $\left.\mathrm{p}^{6}\right] 4 \mathrm{~d}^{9} n \mathrm{p}$ ( $n=5-8), 4 \mathrm{~d}^{9} n f(n=5-7), 4 \mathrm{~d}^{8} 5 \mathrm{~s} 5 \mathrm{p}, 4 \mathrm{~d}^{8} 5 \mathrm{p} 5 \mathrm{~d}, 4 \mathrm{~d}^{8} 4 \mathrm{f} 5 \mathrm{~s}, 4 \mathrm{~d}^{8} 5 \mathrm{~d} n f(n=4,5)$. These sets somewhat extend the ones used by Campos et al. [30] and found by them to produce transition rates and radiative lifetimes that agree well with experiment and other high-quality calculations. All known energy levels from Table 2, except for the questionable $4 \mathrm{~d}^{9} 6 \mathrm{p}^{3} \mathrm{P}^{\circ}$, were included in the least squares fitting (LSF) procedure. In LSF, the average energies of the $4 \mathrm{~d}^{10}, 4 \mathrm{~d}^{9} n \mathrm{~s}(n=5-7)$, and $4 \mathrm{~d}^{9} 5 \mathrm{p}$ configurations were varied independently, while the average energies of the $4 \mathrm{~d}^{9} n \mathrm{~s}(n=8,9), 4 \mathrm{~d}^{9} n \mathrm{~d}(n=5-9)$, $\left[4 \mathrm{~d}^{8} 5 l^{2}(l=\mathrm{s}, \mathrm{p}, \mathrm{d}), 4 \mathrm{~d}^{8} 5 \mathrm{pnf}(n=4,5)\right]$ (even parity), $4 \mathrm{~d}^{9} n \mathrm{p}(n=6-8), 4 \mathrm{~d}^{9} n \mathrm{f}(n=4-8)$, and [4d $\left.{ }^{8} 5 \mathrm{~s} 5 \mathrm{p}, 4 \mathrm{~d}^{8} 5 \mathrm{p} 5 \mathrm{~d}, 4 \mathrm{~d}^{8} 4 \mathrm{f} 5 \mathrm{~s}, 4 \mathrm{~d}^{8} 5 \mathrm{~d} n f(n=4,5)\right]$ (odd parity) configurations were linked together in the corresponding groups with ratios within each group fixed at the Hartree-Fock (HF) values. Thus, the positions of unknown highly excited configurations were scaled according to the experimentally known lowest configuration in each group. Similarly, the values of the $\zeta_{4 \mathrm{~d}}$ parameter for the $4 \mathrm{~d}^{9} n l$ and $4 \mathrm{~d}^{8} n \ln l^{\prime}$ configuration groups were linked together in two groups for each parity 
with ratios fixed at the HF values. Similar linking of the electrostatic parameters $G^{k}(4 \mathrm{~d}, n l)$ and $F^{k}(4 \mathrm{~d}, n l)$ was also enforced along series of configurations. The effective parameter $\alpha_{4 \mathrm{~d}}$ was found to be $38.8(3) \mathrm{cm}^{-1}$ from the fitting of the $4 \mathrm{~d}^{8} 5 \mathrm{~s}^{2}$ configuration and then the same value was used in all other $4 \mathrm{~d}^{8} n \ln l^{\prime}$ configurations of both parities. Very small or poorly defined single-configuration parameters, such as $\zeta_{5 \mathrm{~d}}$ for $4 \mathrm{~d}^{9} 5 \mathrm{~d}$, as well as all configuration-interaction parameters were fixed at the HF level for $\zeta_{n l}$ and $0.8 \times \mathrm{HF}$ for all others.

I fitted 43 known levels of even parity with 17 free parameters, with a standard deviation of $23 \mathrm{~cm}^{-1}$, and 55 odd-parity levels with 18 free parameters, with a standard deviation of $63 \mathrm{~cm}^{-1}$.

In these calculations, I found that the $4 \mathrm{~d}^{9} \mathrm{nl}$ configurations are best described in the $J K$ coupling scheme, in which the purity of levels varies from $79 \%$ for $4 d^{9} 5 p$ to about $95 \%$ for $4 d^{9} 5 d$ and all $4 d^{9} n l$ with $n>5$. Therefore, the configuration and term labels, as well as percentage compositions for these configurations are given in Table 2 in this coupling scheme, providing unambiguous physically meaningful designations. For the $4 d^{8} 5 s 5 p$ configuration, $L S$ coupling of the type $4 d^{8}+5 s 5 p$ was found to give a slightly better purity of levels, $56 \%$, compared to the coupling $4 d^{8} 5 s+5 p, 54 \%$. For some of the levels of this configuration, the leading percentage is as small as $25 \%$. In these cases, the configuration and term labels have little physical meaning and are used for bookkeeping purpose only.

\section{Relative Intensities of Observed Lines}

As noted in the Introduction, the relative intensities of lines observed with different light sources and with different registration equipment are vastly different. In order to give a consistent set of relative intensities, they must be converted to the same scale. To account for the different excitation conditions in various light sources, the observed line intensities can be approximated by local thermodynamic equilibrium (LTE) with an effective excitation temperature pertinent to each light source, and then scaled to the same excitation temperature. In reality, the LTE approximation describes the observed intensities only qualitatively, with deviations in both directions up to an order of magnitude. However, this method results in much better qualitative agreement between relative intensities observed by different authors. In addition to the different effective temperatures in the light sources, the observed intensities are strongly affected by different responses of the dispersive elements and detectors to different wavelengths. These variations can also be accounted for and removed. The general method for doing this was described in my recent paper on In II [31]. It relies on radiative transition rates $A_{k i}$ calculated with Cowan's codes (see the previous section), and on the LTE relation between these transition rates and the observed intensities $I_{\mathrm{obs}}$ :

$$
I_{\mathrm{obs}} \propto\left(g_{k} A_{k i} / \lambda\right) \exp \left(-E_{k} / k T_{\text {eff }}\right),
$$

where $g_{\mathrm{k}}$ and $E_{k}$ are the statistical weight and energy of the upper level, $\lambda$ is the central wavelength of the line in vacuum, $k$ is the Boltzmann constant, and $T_{\text {eff }}$ is the effective temperature.

\section{Transition Probabilities}

Experimental transition probabilities ( $A$-values) for transitions originating from the $4 d^{8} 5 s^{2}, 4 d^{9} 6 s$, and $4 \mathrm{~d}^{9} 5 \mathrm{~d}$ configurations have been obtained by Campos et al. [30] using a combination of theoretical lifetimes and measured branching fractions. The calculations were made with Cowan's codes [29] using two models, one with semi-empirical account for core polarization (CP), and the other one without core polarization corrections, but with an extended set of interacting configurations that effectively introduced the corepolarization effects. In both models, the Slater parameters were adjusted by the LSF method to fit experimental energy levels. The CP calculations were deemed to be more accurate and were used to derive the radiative lifetimes. The accuracy of the calculated lifetimes was indirectly confirmed by a close 
agreement of the lifetimes in the homologous spectrum $\mathrm{Cu}$ II, calculated using a similar method, with experiments.

The radiative lifetimes of the $4 \mathrm{~d}^{9} 5 \mathrm{p}$ levels were measured by Biémont et al. [11] using selective laser excitation of a fast ion beam. These experimental radiative lifetimes were used in that work to renormalize the calculated transition probabilities. In this procedure, the calculated $A$-values for all transitions originating from a common upper level were multiplied by a factor equal to the ratio of the measured and calculated lifetimes. This factor was equal to 1.06 on average. The calculations were made with Cowan's codes [29] using an extended set of interacting configurations (17 of even parity and 13 of odd parity) including configurations with an open $4 p$ shell to mimic the core-polarization effects. The calculated eigenvalues were adjusted to the available observed energy levels by a LSF procedure.

Relative $A$-values of the $4 d^{9} 5 s-4 d^{9} 5 p$ transitions were determined by Ferrero et al. [32] from measurements of emission line intensities in an optically thin laser-produced plasma. They were placed on an absolute scale by normalizing to their own HFR (Hartree-Fock-Relativistic) calculations with Cowan's codes [29]. Ferrero et al. assumed that their HFR calculations are accurate to $5 \%$. However, these calculations were single-configuration and, as evidenced by more extensive calculations of Biémont et al. [11], as well as my own (see Sec. 5), this estimate was far too optimistic. Nevertheless, the experimental results of Ferrero et al. are still useful. They did not give the measured intensity values, but it was possible to deduce them from the given $A$-values using the fact that the LTE condition was experimentally confirmed for the studied plasma source. I renormalized these relative intensities to the lifetimes measured by Biémont et al. [11]. For some radiative branches that were not observed by Ferrero et al. [32], I used calculated $A$-values from Biémont et al. [11]. For five weak transitions, where the uncertainties of the measured branching fractions were too large (from the $4 \mathrm{~d}^{9} 4 \mathrm{p}^{3} \mathrm{D}^{\circ}{ }_{1}$ level), or the radiative lifetime was not reliably measured (from the $4 \mathrm{~d}^{9} 4 \mathrm{p}^{1} \mathrm{P}^{\circ}{ }_{1}$ level), I used the Boltzmann equation (4) to deduce the $A$-values.

It should be noted that all three sources discussed above [11,30,32] used the energy levels from Benschop et al. [5] in their LSF calculations. Although the analysis of the latter authors was incorrect (see Sec. 2.6), the energy levels given by them deviate from the true values by only a few reciprocal centimeters, which does not introduce noticeable errors in calculations of $A$-values.

The $A$-values from the above three sources comprised a sufficiently large set of reference data to estimate the uncertainties of my transition-probability calculations described in Sec. 5. The procedure is illustrated in Fig. 2.

For strong lines (line strength $S>5.65$ ), the average deviation from the reference $A$-values is about $20 \%$. For medium-strength lines $(0.32<S<5.65)$, the average deviation is $32 \%$. I adopt these average deviations as estimates of uncertainties and extrapolate them to other calculated $A$-values with line strengths from the corresponding ranges.

In the shaded region of Fig. 2 (very weak lines with $S<0.32$ ), the calculations are unusable due to large uncertainties (factor of two or greater).

In such comparisons one should use the logarithms of $A$-values or $\log (g f)$ rather than $A$-values themselves, because of statistical properties of quantum-mechanical calculations. For the quantities $\log (A)$ or $\log (g f)$, statistical distribution of deviations from the true values is usually much closer to normal than for the $A$-values, which allows one to use the term "uncertainty" with a well-defined meaning (in the case of the present work, the one-standard-deviation uncertainty of $\log (A)$ is \pm 0.08 for strong lines with $S>5.65$, see Fig. 2).

Together with calculated A-values, Cowan's codes [29] provide values of the cancellation factor CF,

$$
\mathrm{CF}=\left(S^{+}+S^{-}\right) /\left(S^{+}+\left|S^{-}\right|\right),
$$

where $S^{+}$and $S^{-}$are sums of positive and negative contributions to $S$, respectively. Small absolute values of CF indicate that the corresponding $A$-values are unreliable. In the case of my Ag II calculations, a plot similar to Fig. 2, but with CF instead of $S$ on the horizontal axis, reveals that for $|\mathrm{CF}|<0.1$ the calculated $A$ values have uncertainties greater than a factor of 2 . Such $A$-values are not included in Table 1. 


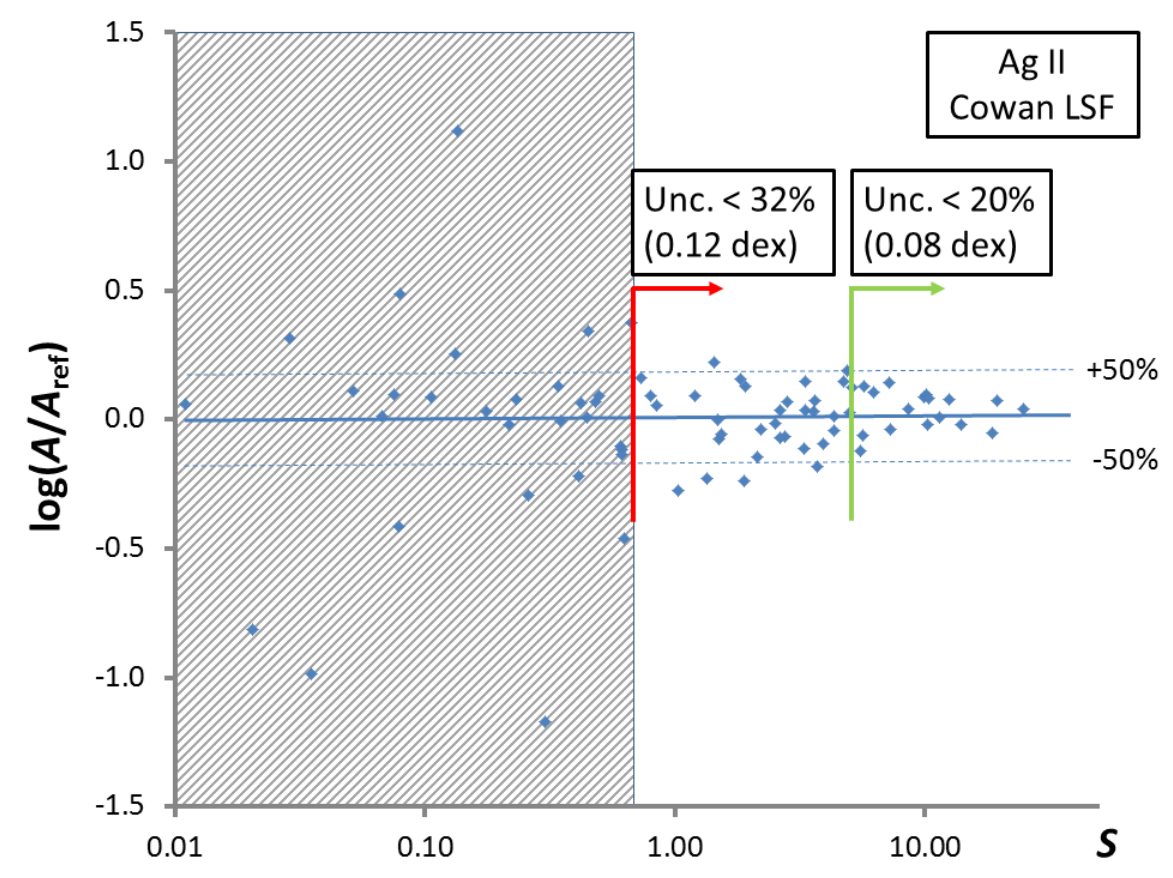

Fig. 2. Estimation of uncertainties of calculated $A$-values for $A g$ II. $S$ is the line strength, and the reference values $A_{\text {ref, }}$ having uncertainties smaller than $20 \%$, are selected from three sources described in the text.

All adopted $A$-values from the three published sources and the present work are given in Table 1. For the reasons discussed above, uncertainties of the adopted $A$-values are specified in Table 1 with a letter code instead of numerical values. The letter code is explained in Table 3.

Table 3. Transition probability uncertainty code

\begin{tabular}{lll}
\hline \hline Symbol & $\begin{array}{l}\text { Uncertainty } \\
\text { in A-value }\end{array}$ & $\begin{array}{l}\text { Uncertainty } \\
\text { in } \log (g f)\end{array}$ \\
\hline A & $\leq 3 \%$ & $\leq 0.013$ \\
B + & $\leq 7 \%$ & $\leq 0.03$ \\
B & $\leq 10 \%$ & $\leq 0.04$ \\
C+ & $\leq 18 \%$ & $\leq 0.08$ \\
C & $\leq 25 \%$ & $\leq 0.11$ \\
D+ & $\leq 40 \%$ & $\leq 0.18$ \\
D & $\leq 50 \%$ & $\leq 0.24$ \\
E & $>50 \%$ & $>0.24$ \\
\hline
\end{tabular}

In addition to the papers discussed above, there are several other publications involving calculated transition probabilities and measured radiative lifetimes in Ag II. They are not discussed here because their accuracy was found to be lower than for the used sources. A complete list of relevant papers can be retrieved from the NIST Atomic Transition Probability Bibliographic Database [33]. 


\section{Conclusions}

In the present paper, critically evaluated energy levels, wavelengths, and transition probabilities have been derived for the Ag II spectrum from available experimental and theoretical data. The level list includes 98 excited energy levels having relative uncertainties ranging from $0.0012 \mathrm{~cm}^{-1}$ to $0.24 \mathrm{~cm}^{-1}$. The uncertainty of the connection between the excited level system and the ground level is $0.06 \mathrm{~cm}^{-1}$. The line identifications and level values of the $4 \mathrm{~d}^{9} 9$ s configuration from the work of Benschop et al. [5] have been found incorrect. A revised value of the first ionization limit, 173283(7) $\mathrm{cm}^{-1}$, equivalent to $21.4844(8) \mathrm{eV}$, has been derived from four $4 \mathrm{~d}^{9} n \mathrm{~s}^{1,3} \mathrm{D}$ Rydberg series $(n \leq 8)$. The complete line list includes 452 observed spectral lines between $728 \AA$ and $9053 \AA$. Transition probabilities have been critically assessed for 237 transitions; 141 of them have been calculated in the present work. Uncertainties of the assessed transition probabilities range from $2 \%$ to $75 \%$; for 226 transitions, the uncertainties are smaller than 35\%.

\section{Acknowledgments}

This work is supported in part by the U. S. National Aeronautics and Space Administration.

\section{References}

[1] G. Kalus, U. Litzén, F. Launay, and W.-Ü L. Tchang-Brillet, Phys. Scr. 65, 46 (2002). http://dx.doi.org/10.1238/physica.regular.065a00046

[2] W. P. Gilbert, Phys. Rev. 47, 847 (1935). http://dx.doi.org/10.1103/PhysRev.47.847

[3] E. Rasmussen, Phys. Rev. 57, 840 (1940). http://dx.doi.org/10.1103/PhysRev.57.840

[4] E. Rasmussen, K. Dan, Vidensk. Selsk., Mat.-Fys. Medd. 18 (5), 1-32 (1940).

[5] H. Benschop, Y. N. Joshi, and Th. A. M. van Kleef, Can. J. Phys. 53, 700 (1975). http://dx.doi.org/10.1139/p75-088

[6] A. G. Shenstone, Phys. Rev. 31, 317 (1928). http://dx.doi.org/10.1103/PhysRev.31.317

[7] H. A. Blair, Phys. Rev. 36, 173 (1930). http://dx.doi.org/10.1103/PhysRev.36.173

[8] R. Engleman, Jr., U. Litzén, H. Lundberg, and J.-F. Wyart, Phys. Scr. 57, 345 (1998). http://dx.doi.org/10.1088/0031-8949/57/3/006

[9] A. Kramida, Yu. Ralchenko, J. Reader, and NIST ASD Team (2012), NIST Atomic Spectra Database (ver. 5.0); available at http://physics.nist.gov/asd.

[10] J. E. Sansonetti and W. C. Martin, Handbook of Basic Atomic Spectroscopic Data, J. Phys. Chem. Ref. Data 34, 1559 (2005); available at: http://physics.nist.gov/PhysRefData/Handbook/index.html. http://dx.doi.org/10.1063/1.1800011

[11] E. Biémont, E. H. Pinnington, J. A. Kernahan, and G. Rieger, J. Phys. B 30, 2067 (1997). http://dx.doi.org/10.1088/0953-4075/30/9/009

[12] E. R. Peck and K. Reeder, J. Opt. Soc. Am. 62, 958 (1972). http://dx.doi.org/10.1364/JOSA.62.000958

[13] G. Norlén, Phys. Scr. 8, 249 (1973). http://dx.doi.org/10.1088/0031-8949/8/6/007

[14] G. Nave and C. J. Sansonetti, J. Opt. Soc. Am. B 28, 737 (2011). http://dx.doi.org/10.1364/JOSAB.28.000737

[15] A. E. Kramida and G. Nave, Eur. Phys. J. D 39, 331 (2006). http://dx.doi.org/10.1140/epjd/e2006-00121-4

[16] A. E. Kramida, Comput. Phys. Commun. 182, 419 (2011). http://dx.doi.org/10.1016/j.cpc.2010.09.019

[17] F. Exner and E. Haschek, Die Spektren der Elemente bei normalem Druck, Franz Deuticke, Leipzig und Wien (1911).

[18] J. Frings, Zeits. Wiss. Photog. 15, 165 (1915).

[19] H. Benschop, Y. N. Joshi, and Th. A. M. van Kleef, Can. J. Phys. 53, 498 (1975). http://dx.doi.org/10.1139/p75-063

[20] Y. N. Joshi, V. S. Kushawaha, H. Benschop, and Th. A. M. van Kleef, Can. J. Phys. 53, 1689 (1975). http://dx.doi.org/10.1139/p75-215

[21] R. D. Reid, D. C. Gerstenberger, J. R. McNeil, and G. J. Collins, J. Appl. Phys. 48, 3994 (1977). http://dx.doi.org/10.1063/1.324236

[22] R. D. Reid, W. L. Johnson, J. R. McNeil, and G. J. Collins, IEEE J. Quantum Electron. 12, 778 (1976). http://dx.doi.org/10.1109/JQE.1976.1069088

[23] J. R. McNeil, W. L. Johnson, G. J. Collins, and K. B. Persson, Appl. Phys. Lett. 29, 172 (1976). http://dx.doi.org/10.1063/1.89012

[24] W. L. Johnson, J. R. McNeil, G. J. Collins, and K. B. Persson, Appl. Phys. Lett. 29, 101 (1976). http://dx.doi.org/10.1063/1.88984

[25] G. R. Harrison, M.I.T. Wavelength Tables, 1969 Edition, MIT Press, Cambridge, Massachusetts, 429 pp. (1969); F. M. Phelps, III, M.I.T. Wavelength Tables, Vol. 2, MIT Press, Cambridge, Massachusetts, 810 pp. (1982).

[26] C. E. Moore, Atomic Energy Levels as Derived from the Analysis of Optical Spectra - Molybdenum through Lanthanum and Hafnium through Actinium, Nat. Bur. Stand. Circ. 467, Vol. III (1958); Reprinted as Nat. Stand. Ref. Data Ser., Nat. Bur. Stand. (U.S.) 35, Vol. III (1971), 245 pp. 
[27] C. Sansonetti, Fortran computer code RITZPL (private communication, 2005).

[28] M. A. Baig, A. Rashid, Z. Iqbal, and J. Hormes, J. Phys. B 24, 2295 (1991). http://dx.doi.org/10.1088/0953-4075/24/9/009

[29] R. D. Cowan, The Theory of Atomic Structure and Spectra, University of California Press, Berkeley, CA, 731 pp. (1981); A version of the codes adapted for Windows-based computers by A. Kramida is available online at http://das101.isan.troitsk.ru/COWAN.

[30] J. Campos, M. Ortiz, R. Mayo, E. Biémont, P. Quinet, K. Blagoev, and G. Malcheva, Mon. Not. R. Astron. Soc. 363, 905 (2005). http://dx.doi.org/10.1111/j.1365-2966.2005.09493.x

[31] A. Kramida, Res. J. Nat. Inst. Stand. Technol. 118, 52 (2013). http://dx.doi.org/10.6028/jres.118.004

[32] F. S. Ferrero, C. Cerezo, M. J. F. Cigoña, and J. Campos, J. Quant. Spectrosc. Radiat. Transfer 54, 971 (1995). http://dx.doi.org/10.1016/0022-4073(95)00120-A; See also F. S. Ferrero et al., Erratum, J. Quant. Spectrosc. Radiat. Transfer 55, 533 (1996). http://dx.doi.org/10.1016/0022-4073(96)80773-4

[33] A. Kramida and J. R. Fuhr, NIST Atomic Transition Probability Bibliographic Database, v. 9.0 (2012); available at http://physics.nist.gov/fvalbib.

About the author: Alexander Kramida is a physicist in the Atomic Spectroscopy Group of the Quantum Measurement Division, Physical Measurement Laboratory at the National Institute of Standards and Technology, Gaithersburg, MD. The National Institute of Standards and Technology is an agency of the U.S. Department of Commerce. 